\title{
MANIPULATING NEURONAL COMMUNICATION BY USING LOW-INTENSITY REPETITIVE TRANSCRANIAL MAGNETIC STIMULATION COMBINED WITH ELECTROENCEPHALOGRAPHY
}

\author{
Dissertation \\ for the award of the degree \\ Doctor rerum naturalium \\ of the Georg-August-Universität Göttingen \\ within the doctoral program Theoretical and Computational Neuroscience \\ of the Georg-August University School of Science (GAUSS) \\ submitted by \\ Elina Zmeykina
}

from Chistopol, Russia

Göttingen, 2021 


\section{Thesis Committee:}

Prof. Dr. med. Walter Paulus (Supervisor, First Referee)

Georg-August-Universität Göttingen, Klinik für klinische Neurophysiologie, Universitätsmedizin Göttingen

\section{Prof. Dr. Florentin Wörgötter (Second Referee)}

Georg-August University Göttingen, Third Institute of Physics - Biophysics

\section{Dr. Andreas Neef}

Campus Institute for Dynamics of Biological Networks, Max-Planck-Institute for Experimental Medicine

\section{Members of the Examination Board:}

\section{Prof. Dr. Melanie Wilke}

Department of Cognitive Neurology, University Medical Center Göttingen

\section{Dr. Roberto Goya-Maldonado}

Systems Neuroscience and Imaging in Psychiatry Lab, University Medical Center Göttingen

\section{Prof. Dr. Hannelore Ehrenreich}

Clinical Neuroscience, Max Planck Institute of Experimental Medicine

Date of thesis submission: 08.02.2021

Date of oral examination: 23.03.2021 


\begin{abstract}
s
Repetitive transcranial magnetic stimulation (rTMS) modulates ongoing brain rhythms by activating neuronal structures and evolving different neuronal mechanisms. In the current work, the role of stimulation strength and frequency for brain rhythms was studied. We hypothesized that a weak oscillating electric field induced by low-intensity rTMS could induce entrainment effects in the brain. To test the hypothesis, we conducted three separate experiments, in which we stimulated healthy human participants with rTMS. We individualized stimulation parameters using computational modeling of induced electric fields in the targets and individual frequency estimated by electroencephalography (EEG). We demonstrated the immediately induced entrainment of occipito-parietal and sensorimotor mu-alpha rhythm by low-intensity rTMS that resulted in phase and amplitude changes measured by EEG. Additionally, we found long-lasting corticospinal excitability changes in the motor cortex measured by motor evoked potentials from the corresponding musle.
\end{abstract}




\section{Table of content}

1. LIST OF ABBREVIATIONS

2. INTRODUCTION 9

2.1. BRAIN RHYTHMS $\quad 10$

2.2. BIOPHYSICS OF BRAIN OSCILLATIONS 11

2.3. ROLE OF $\mu$-ALPHA RHYTHM 12

2.4. THE PHYSICAL CONCEPT OF OSCILLATIONS 14

2.5. TARGETING RHYTHMS BY NIBS 17

2.6. BASIC PRINCIPLES OF TRANSCRANIAL MAGNETIC STIMULATION 18

2.7. NEURAL ENTRAINMENT EFFECT INDUCED BY TMS 19

2.8. LIMITATION OF THE TMS TECHNIQUE $\quad 20$

2.9. OBJECTIVES AND STRUCTURE OF THIS DISSERTATION 21

3. WEAK RTMS-INDUCED ELECTRIC FIELDS PRODUCE NEURAL ENTRAINMENT IN HUMANS

3.1. ABSTRACT 24

3.2. INTRODUCTION 25

3.3. RESUlts 28

3.3.1. STUDY OVERVIEW 28

3.3.2. RHYTHMIC RTMS SYNCHRONIZES ONGOING POSTERIOR ALPHA RHYTHMS 30

3.3.3. RTMS INDUCES RAPID AND SUSTAINED INCREASE IN THE ONGOING POSTERIOR ALPHA SYNCHRONIZATION 36

3.3.4. MEAN AND MEDIAN ELECTRIC FIELD STRENGTHS ARE IN THE EFFECTIVE RANGE 39

3.3.5. IMMEDIATE EFFECTS IN THE RANGE OF 30 TO 42\% OF THE RESTING MOTOR THRESHOLD 41

3.4. DISCUSSION 42

3.4.1. COMPARING OUR RESULTS WITH PREVIOUS ELECTROPHYSIOLOGICAL FINDINGS 43

3.4.2. RELATION OF ELECTRIC FIELD STRENGTHS TO PREVIOUS TACS AND RTMS STUDIES 44

3.4.3. TOWARDS A BETTER UNDERSTANDING OF THE NEURAL MECHANISMS OF RTMS 45

3.4.4. FUTURE DIRECTIONS 46

3.5. METHODS

3.5.1. PARTICIPANTS

3.5.2. ETHIC STATEMENT AND RESEARCH INTEGRITY 48

3.5.3. Procedure 48

3.5.4. ACQUISITION AND ANALYSIS OF NEUROIMAGING DATA 49

3.5.5. NEURONAVigATED (R)TMS 
3.5.6. DETERMINATION OF MOTOR THRESHOLD $\quad 52$

3.5.7. HEAD MODELING AND EF CALCULATIONS

3.5.8. EEG ACQUISITION

3.5.9. ESTIMATING INDIVIDUAL ALPHA BAND PEAK FREQUENCIES

3.5.10. SimultaneOUS RTMS AND EEG 56

3.5.11. ANALYSIS OF RTMS-EEG 58

3.6. SUPPLEMENTARY MATERIAL 61

3.6.1. VALIDATION MEASUREMENTS 61

4. SHORT-LIVED ALPHA POWER SUPPRESSION INDUCED BY LOW-INTENSITY ARRHYTHMIC RTMS $\quad 66$

4.1. ABSTRACT 167

4.2. INTRODUCTION

4.3. METHODS $\quad 69$

4.3.1. SECONDARY ANALYSIS $\quad 69$

4.3.2. PARTICIPANTS

$\begin{array}{ll}\text { 4.3.3. ETHICS } & 70\end{array}$

4.3.4. HEAD MODELING AND ELECTRIC FIELD ESTIMATION 70

4.3.5. EXPERIMENTAL PROCEDURE AND STIMULATION PARAMETERS 70

4.3.6. EEG ANALYSIS

4.4. RESUlts

4.4.1. SHORT-TERM AFTEREFFECT

4.4.2. LONG-TERM AFTEREFFECT

4.5. DISCUSSION 80

4.6. SUPPLEMENTAL INFORMATION 83

5. INHIBITING CORTICOSPINAL EXCITABILITY BY ENTRAINING ONGOING $\mu$ ALPHA RHYTHM IN MOTOR CORTEX

5.1. ABSTRACTS

5.2. INTRODUCTION

5.3. METHODS $\quad 88$

5.3.1. PARTICIPANTS $r 8$

5.3.2. OVERVIEW OF THE EXPERIMENTAL SESSIONS 199

5.3.3. TMS AND NEURONAVIGATION 9

5.3.4. RTMS PROTOCOLS 91

5.3.5. HEAD MODELING AND EF CALCULATIONS 92

5.3.6. MRI AND FMRI DATA ACQUISITION 93

5.3.7. EMG ACQUISITION 93 
5.3.8. RTMS-EEG DATA ACQUISITION

5.3.9. DATA ANALYSIS 95

5.3.10. RTMS-EEG ANALYSIS

5.4. RESULTS 99

5.4.1. INCREASED MU-ALPHA SYNCHRONIZATION DURING AND AFTER RHYTHMIC RTMS 99

5.4.2. RHYTHMIC RTMS INCREASED ALPHA/BETA POWER AFTER STIMULATION 101

5.4.3. RHYTHMIC RTMS DECREASED CORTICOSPINAL EXCITABILITY LEVEL 103

5.5. DISCUSSION 104

5.5.1. ELECTROPHYSIOLOGICAL EFFECTS OF LOW-INTENSITY RTMS 105

5.5.2. ARRHYTHMIC RTMS INDUCES ALPHA PERTURBATION IN THE OCCIPITAL BUT NOT THE MOTOR CORTEX. 106

5.5.3. LOW-INTENSITY RTMS AFFECTS CORTICOSPINAL EXCITABILITY 107

5.6. CONCLUSIONS 108

6. GENERAL DISCUSSION 101

$\begin{array}{ll}\text { 7. SIGNIFICANCE AND CONCLUSION } & 105\end{array}$

8. BIBLIOGRAPHY 106

9. LIST OF FIGURES 123

10. LIST OF TABLES 124

11. ACKNOWLEDGMENTS 125

12. DECLARATION OF CONTRIBUTION 126

13. CURRICULUM VITAE 127 


\section{List of Abbreviations}

act

AMT

CSF

DLPFC

EEG

EEG RoT

$\mathrm{EF}, \mathrm{EF}$

EPI

ERP

FDI

FEM

fMRI

FOV

FXD

GM

IAF

ICA

iIPS

IMF

IPL

ITI

LCMV

M1

MEG

MEP

MNI

MRI

$\mathrm{MSO} \%$ active/real stimulation

active motor threshold

cerebrospinal fluid

dorsolateral prefrontal cortex

electroencephalography

EEG electrode landmark and rule of thumb

electric field

echo-planar imaging

event-related potential

first dorsal interosseous muscle

finite element model

functional magnetic resonance imaging

field of view

fixed intensity

gray matter

individual alpha frequency

independent component analysis

inferior intraparietal sulcus

individual mu frequency

inferior parietal lobe

inter-train interval

linear constrained minimum variance

primary motor cortex

magnetoencephalography

motor evoked potential

Montreal Neurological Institute coordinate space

magnetic resonance imaging

percentage of maximum stimulator output 


$\begin{array}{ll}\text { MT } & \text { motor threshold } \\ \text { NIBS } & \text { non-invasive brain stimulation } \\ \text { n.s. } & \text { not significant } \\ \text { PLV } & \text { phase-locking value } \\ \text { PMID } & \text { PubMed identification number } \\ \text { PT } & \text { phosphene threshold } \\ \text { REM } & \text { rapid-eye movement } \\ \text { RMD } & \text { repeated-measures design } \\ \text { RMT } & \text { resting motor threshold } \\ \text { ROI } & \text { region of interest } \\ \text { rTMS } & \text { repetitive transcranial magnetic stimulation } \\ \text { S1 } & \text { primary somatosensory cortex } \\ \text { SGD } & \text { separate group design } \\ \text { sh } & \text { sham } \\ \text { SPL } & \text { repetition time } \\ \text { spTMS } & \text { visual motor threshold } \\ \text { tACS } & \text { white matter } \\ \text { TE } & \text { transcranial alternating current stimulation } \\ \text { TR } & \text { echo time } \\ \text { vMT } & \text { WM }\end{array}$




\section{Introduction}

Neural oscillations are spatiotemporally structured activity patterns that form a hierarchically organized system in the cerebral cortex. A rhythmic pattern of neurons activated on the same timescale can occur locally (within a specific brain area) or globally (between brain areas). Oscillations have an essential role in computation and diverse brain functions.

Non-invasive brain stimulation (NIBS) offers the opportunity to interact with ongoing oscillations by manipulating the frequency, intensity, and stimulation location. Repetitive transcranial magnetic stimulation (rTMS) is a form of NIBS technique based on delivering repetitive brief magnetic pulses, which can interact with underlying cortical neurons' ongoing activity by inducing an electric field (EF) in them. While rTMS has shown therapeutic benefits for psychiatric and neurological diseases, such as addiction, depression, Parkinson's disease, schizophrenia, and stroke ${ }^{1}$, the cellular and neural mechanisms remain poorly understood ${ }^{2,3}$.

In this doctoral dissertation, I studieded the entrainment effects induced by periodic pulses in humans. Specifically, the thesis aims were 1) to develop a prospective EF estimation approach of rTMS intensity selection for effectively interact with neuronal oscillations by rTMS and 2) to study its immediate and long-lasting electrophysiological and neurophysiological effects accessed by electroencephalography (EEG) and corticospinal excitability changes.

In the following, I outline the theoretical background and current state of TMS techniques and their combination with EEG to study the neural mechanisms underlying cortical brain rhythms. Chapter 2 and Chapter 3 focus on validating a prospective EF estimation approach and parameters of rTMS to obtain dose/response information crucial for entraining occipito-parietal oscillations in resting-state and exploring the neural mechanisms of these entrainments. Chapter 4 investigates 
the modulation of the $\mu$-alpha oscillations in the motor cortex and its functional effect on motor cortex excitability. Last, Chapter 5 attempted to explain the induced effects by the theoretical framework of rhythm's synchronization.

\subsection{Brain rhythms}

Oscillations are abundant in nature. All fundamental processes in nature, such as the daynight cycle or the sun activity, have periodical essence. Biological systems, for example, circadian rhythm, also follow rhythmic patterns, and brain activity is not an exception. Neural oscillations provide an insight into the computational principles of neural activity. Distant brain regions require a communication tool to process information and execute behavior effectively.

It has been a century now since the German neurologist Hans Berger first observed the rhythmic pattern of the EEG recorded from the human scalp. In recent years, the rhythmic fluctuations have been linked to behavioral and cognitive processes. Electrophysiological studies in animals and humans have shown that distributed synchrony over distinct cortical regions correlates with the various neural computations that underlie perception, cognition, and action ${ }^{4-8}$. However, in most studies, the relation between oscillations and cognition is only correlative. Direct causative proof that changes in synchronous activity can affect behavior remains to be established.

Classically, five frequency bands are experimentally observed in humans (delta, $0.5-4 \mathrm{~Hz}$; theta, 4-7 Hz; alpha, $8-13 \mathrm{~Hz}$; beta, $14-30 \mathrm{~Hz}$; gamma, $>30 \mathrm{~Hz}$ ). However, the separation is partly arbitrary. The same neural mechanism might give rise to different frequency bands, or different mechanisms might result in the same oscillation frequency of neural assemblies. In this doctoral 
dissertation, I focus on two rhythms within the $8-12 \mathrm{~Hz}$ range that are spatially distinguished into sensorimotor $\mu$ and occipito-parietal alpha rhythms.

The synchronized brain activity patterns have also been observed at different spatial scales, from a single neuron resonating at multiple frequencies to widespread networks. For example, neurons in the cat visual cortex, separated only by ca. $5 \mathrm{~mm}$, are synchronized in the beta and gamma frequency ranges during REM sleep and wakefulness ${ }^{9}$. At the same time, experiments with electrodes implanted in several more distinct cortical regions of the cat brain demonstrated the large-scale synchronization between the visual and parietal cortex and between the parietal and motor cortex during sensorimotor tasks ${ }^{10}$. Such evidence for large-scale synchronization was also demonstrated with EEG in humans. For example, consistent synchronization of gamma activity between occipital, parietal, and frontal cortical areas was observed during a face recognition $\operatorname{task}^{11}$.

\subsection{Biophysics of brain oscillations}

Different biophysical mechanisms produce oscillatory patterns in the brain. All active neuronal processes such as synaptic activity, action potentials, calcium spikes, intrinsic currents, and other non-synaptic sources generate a potential $\mathrm{V}_{\mathrm{e}}$ with respect to a reference potential. The difference in potentials between two locations superimposes an EF defined as a negative spatial gradient of $\mathrm{V}_{\mathrm{e}}^{12}$. EEG studies investigating the role of brain oscillations in human behavior focus on the synaptic currents and calcium spikes. They are the most important sources of the effects, which EEG can register. An oscillatory cycle begins when extracellular currents from hundreds to tens of thousands of individual synaptic currents overlap in time. Excitatory pyramidal neurons become increasingly active due to reciprocal interaction between each other; this results in a 
general rise of an EF potential. However, they also excite interconnected inhibitory interneurons, which act as an inhibition force for the excitatory neurons, resulting in a fall of the EF potential. As the excitatory drive to interneurons wanes, the neuronal assembly recovers from inhibition, and the next cycle starts again. In other words, several neurons that are close to each other and simultaneously active thus generate EFs that sum together and oscillate in strength. This synchronous activity is strong enough to be measured on the scalp level utilizing EEG.

\subsection{Role of $\mu$-alpha rhythm}

Alpha rhythm reflects a single neuron's fluctuation and their assemblies in the range of 8-13 Hz. It is dominant in the parieto-occipital cortex and is involved in several essential brain functions such as sensory and cognitive processes ${ }^{13}$. Despite decades of research on alpha activity in humans, its physiological pacemaker remains unclear. Several distinct mechanisms such as thalamocortical loops, pyramidal cells' rhythmicity, or local interneurons could generate alpha oscillations ${ }^{14-16}$. Thus, the alpha rhythm is not a unitary phenomenon that originates from a particular single pacemaker. Although specific thalamic nuclei might have a strong influence on cortical alpha oscillations, the variability of amplitude and dynamic in different cortical regions support the assumption that there are many independent alpha generators in the brain ${ }^{17,18}$. A recent study with simultaneous intracranial recordings from the human cortex and thalamic pulvinar demonstrated that alpha oscillations propagate from higher-order (middle temporal, V3) to lower-order (V1 and V2) cortical regions and from cortex to thalamus, contrary to the prevalent theory of a thalamus pacemaker $^{19}$.

Furthermore, cortical alpha oscillations can be registered in all layers of the neocortex with similar spontaneous alpha activity distribution over all cortical layers in the primary visual 
somatosensory and auditory cortex. However, when electrical microstimulation is applied to the somatosensory cortex, unlike in the visual cortex, there is no prominent alpha peak response to stimulation $^{18}$. On the other hand, similarly to occipito-parietal alpha, somatosensory $\mu$ rhythm has been shown to modulate cued attention ${ }^{20}$, supporting the idea that somatosensory $\mu$ and occipitoparietal alpha rhythms are functionally analogous.

(a) Task relevance, timing, and inhibition: possible physiological mechanisms
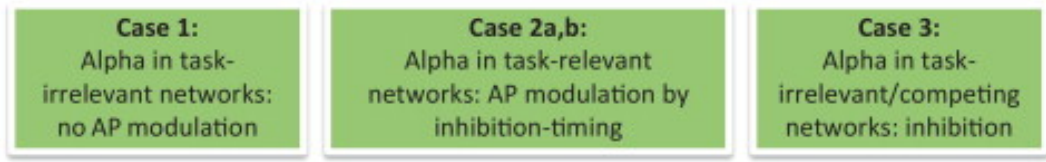

(a)

(b)

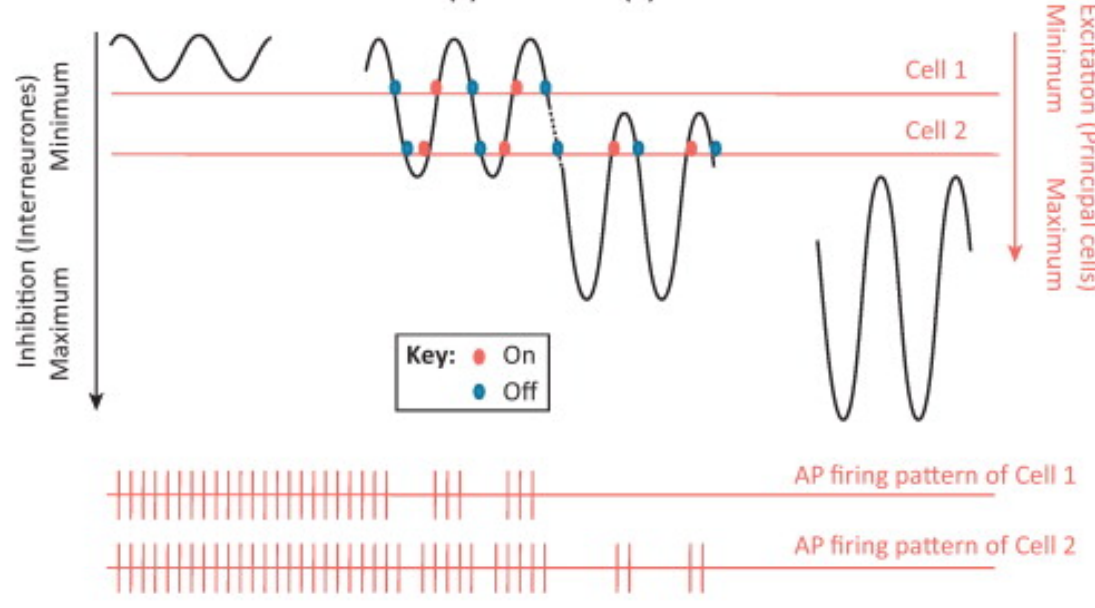

Figure 1. Illustration of inhibition timing hypothesis of alpha oscillations, modified from Figure 1 21 . Besides the case when the amplitude of the alpha rhythm is low, the hypothesis describes two possible scenarios shown in the figure related to the defined timing of cell firing (Case 2a,b) and inhibitory state of cells (Case 3).

The functions of alpha rhythm are principally associated with the brain states at reduced information processing ${ }^{22}$. Usually, alpha is considered an "idling rhythm" ${ }^{23}$, and a higher amplitude is observed when subjects are not involved in any active tasks ${ }^{24}$. The idea of the correlation between alpha rhythm and inhibited or suppressed activity brain states was formulated in the inhibition 
timing hypothesis ${ }^{25}$. Based on brain oscillations' biophysical mechanisms, the hypothesis distinguishes two possible alpha generation mechanisms shown in Fig. 1 (Case 2,3). The amplitude of alpha oscillations is associated with inhibitory cells (interneurons) activation that also suppresses action potentials in the target cell. Depending on the alpha rhythm's task-relevance, the inhibitory network either defines the timing of action potentials or oscillates with a high amplitude suppressing the action potentials completely.

Aberrant alpha patterns were also found in several neurological and psychiatric disorders in which inhibitory regulation is lacking. Several EEG studies demonstrated an increase in alpha power over parietal or frontal regions as well as an asymmetry between the hemispheric alpha activity in clinical populations of patients with depressive syndromes ${ }^{26-28}$, decreased occipitoparietal and resting-state alpha power over central regions in patients with bipolar disorders ${ }^{13,29}$ and decreased alpha power in patients with the obsessive-compulsive disorder ${ }^{30,31}$. A decrease in power and synchronization of alpha oscillations have also been associated with neurodegenerative dementias and mild cognitive impairment such as Alzheimer's disease ${ }^{32,33}$. Regarding the technical

Alpha rhythm has a large amplitude ranging from 10 to $50 \mu \mathrm{V}^{34}$ and the best test-retest reliability than the other EEG rhythms ${ }^{35}$. Altogether, alpha rhythm might be a potential target for therapeutic intervention.

\subsection{The physical concept of oscillations}

Neuronal oscillations are a complex non-linear dynamical system. Nevertheless, the principles that govern their operation follow the fundamental principles of oscillators in other physical systems. The properties of the system themselves entirely determine the brain rhythms. They are maintained due to an internal source of energy and occur spontaneously without an 
external activity. Such oscillators are called autonomous or self-sustained and can be described within a class of nonlinear models. Even though there is evidence to support the view that neural oscillations are, for the most part, non-sinusoidal ${ }^{36}$, nearly all EEG spectral analysis methods are based on the assumption that oscillatory brain activity can be characterized as a sinusoidal wave $\mathrm{e}^{37}$. The neural oscillator is considered as a quasilinear oscillator (the form of an oscillator is close to sinusoidal wave) and described with an equation ${ }^{38}$,

$x(t)=A \sin \left(\omega_{0} t+\varphi_{0}\right)$.

Here, $\omega_{0}$ is a natural frequency $\left(\omega_{0}=2 \pi / T\right.$, where $T$ is a period). The phase of an oscillator is defined as follow:

$$
\varphi(t)=\omega_{0} t+\varphi_{0}
$$

Classical phase synchronization originates from the weak bidirectional coupling of two selfsustained oscillators. Effective communication between brain areas depends on the phase relation between signals of those neural assemblies. Classical entrainment is unidirectional in which the external periodic force is acting on self-sustained oscillators but not vice versa. In the context of this thesis, the entrainment of EEG rhythms is defined as an alignment of the EEG phase to the externally applied oscillating force induced by rTMS, which is defined as

$$
e(t)=\varepsilon \cos \left(\omega t+\overline{\varphi_{e}}\right)
$$

where $\phi_{e}(t)=\omega t+\overline{\varphi_{e}}$ - is the phase of the force, $\varepsilon$ is its amplitude (intensity).

The phases of oscillator and external force do not always match, and their difference is called detuning. The oscillating system's response to external perturbation is described by the function of detuning and intensity of external force shown in Fig. 2. The oscillator is entrained to an external 
force when their phase differences stay constant over time. It can be estimated as a phase-locking value (PLV). The model of Arnold's tongue defines the triangular region (Fig. 2, pink region) in which the entrainment occurs. The ongoing rhythm synchronizes its phase to the external force when their frequencies are matched.

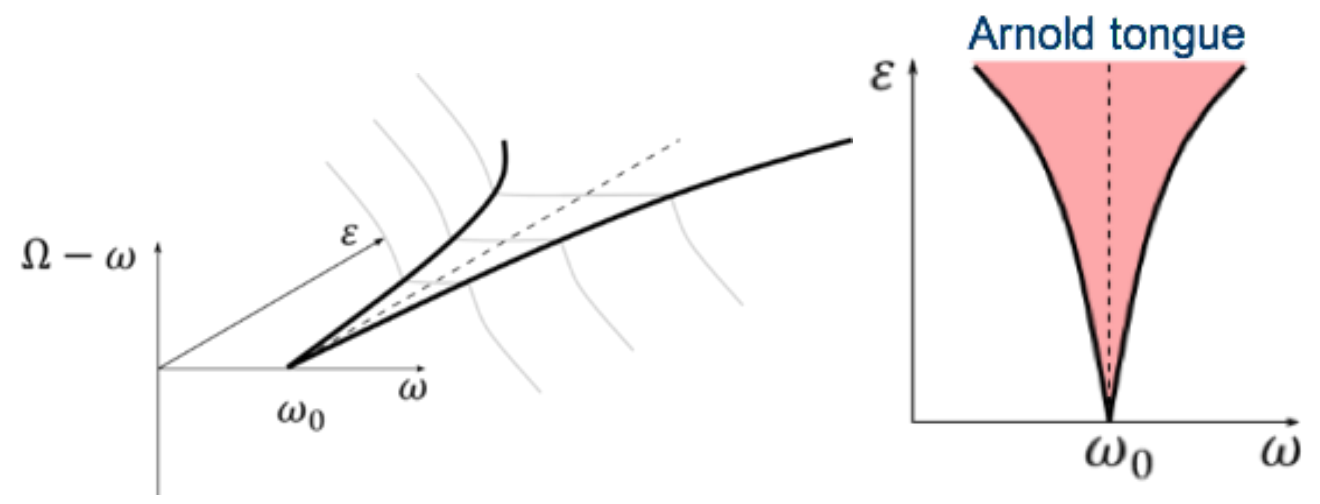

Figure 2. The Arnold tongue model defines the region in which synchronization occurs. A) The family of $\Omega-\omega v$ s. $\omega$ plots for different values of the driving amplitude $\varepsilon$ determines the domain where the frequency of the driven oscillator $\Omega$ is equal to that of the drive $\omega$. This domain, shown by pink in B), is known as the synchronization region or Arnold tongue, adapted from Figure $3.7^{38}$.

The scale of intensities (EFs) range in the context of brain oscillations remains arbitrary because EF's strength defines the induced neural mechanisms. Thus, it is essential to distinguish the entrainment effect from other mechanisms such as resonance effects or spikes bias ${ }^{39}$. There are several requirements necessary to induce neural entrainment ${ }^{40}$ :

1) The presence of the source of the neural oscillator that can oscillate at a given frequency.

2) The periodicity of the externally applied pulses.

3) Synchronization of phase alignment between oscillator and external pulses. 
4) The external force influences the oscillating elements by direct interaction.

The range of EF strength necessary for entrainment has not been specified yet, and its experimental determination became an objective of this dissertation. The induced effects described in this work followed the basic physical principles and the theoretical model of neural entrainment.

\subsection{Targeting rhythms by NIBS}

NIBS induces EFs, which act as an external force for neural entrainment. Brief magnetic pulses or sinusoidal electrical current alter neuronal activity that may facilitate or inhibit their behavior. Computational and experimental studies describe the brain response model to the applied stimulation, but there is no unified physiological determination of parameters that define the induced neuronal mechanisms.

Whereas the frequency is clearly defined in the Arnold tongue model, the induced EF's strength is defined only indirectly. However, the EF purportedly governs the underlying neural mechanisms and functional effects of NIBS. However, due to neuronal populations' anatomical complexity and limited information about alpha generators, induced EFs' values can only be schematically simplified from the theoretical and animal studies ${ }^{39}$. For example, it is hypothesized that inducing an immediate entrainment effect requires a stronger EF (around 2-4 $\mathrm{mV} / \mathrm{mm}$ ) than stochastic resonance or temporal spiking because the endogenous rhythmic activity interferes with the exogenous $\mathrm{EFs}^{41}$. Electrical stimulation, for example, tACS, is applied at an intensity less than $2 \mathrm{~mA}$ peak-to-peak because $1 \mathrm{~mA}$ or higher intensities induce cutaneous side-effects (burning, itching) or phosphenes ${ }^{42}$, which is not well-tolerated by the participants. $3 \mathrm{~mA}$ tACS induces less than $1 \mathrm{mV} / \mathrm{mm} \mathrm{EF}$ in the brain ${ }^{43}$, which is not enough to induce neural entrainment effect; therefore, inducing EF indirectly by TMS is more feasible for inducing the neural entrainment effects. 


\subsection{Basic principles of transcranial magnetic stimulation}

The basic principles of TMS are based on the fundamental law of electromagnetic induction. A strong and transient magnetic field or pulse is delivered through a coil to induce a transitory EF in the underlying cortical surface. In turn, the EF causes the above-threshold depolarization of cell membranes that respond to the currents ${ }^{44,45}$, resulting in transsynaptic repolarization or hyperpolarization of interconnected neurons. Therefore, TMS induces a current that elicits action potentials in neurons. TMS activates all neurons in the stimulation volume, which do not uniformly respond to the applied EF. In general, the EF strength exponentially decays as a function of the distance; therefore, the strongest EF components occur on the crown of the gyrus.

Nevertheless, according to the cortical column cosine model, only the neuron and axons spaced along the EF vector can be activated; therefore, the effective E-filed corresponds to the tangential component, and the highest values occur in the gyral wall ${ }^{46}$. The model emphasizes the importance of the coil orientation for TMS supporting the idea that besides the brain state, TMSinduced response strongly depends on TMS coil geometry ${ }^{47,48}$, individual anatomy of a target subject $^{49,50}$, TMS pulse waveforms, and current directions ${ }^{51-54}$. Early modeling studies utilized spherical models or simplified sulcus geometry ${ }^{55,56}$, although the resolution is insufficient to establish the cortical organization.

Optimizing the TMS parameters by computational modeling and individualizing the stimulation approach allows to decrease the variability of evoked response and opens a new insight into the underlying neural mechanisms. New MRI-based approaches can localize TMS-induced distributed EF with high accuracy ${ }^{49,50,57}$, for example, linking the EF estimations in the motor cortex to the MEP responses allowed to localize the origin of the MEP. Computed normal and 
tangential EF components with finite element models (FEM) revealed the neural structures related to MEP in the gyral crown and the upper parts of the sulcal wall of the motor cortex ${ }^{57}$.

\subsection{Neural entrainment effect induced by TMS}

TMS can be delivered as a strong single pulse at a specific time to probe the excitability of the cortical surface or as a series of repeated pulses in a protocol of $\mathrm{rTMS}^{58}$. Both types of stimulation can modulate the timing of ongoing activity. For example, single-pulse TMS applied to the occipital cortex induces a strong EEG response in the alpha frequency range, whereas stimulation of the somatosensory/parietal cortex results in an EEG response in the beta frequency range $^{59}$. RTMS applied at the natural frequency is shown to synchronize the ongoing rhythm ${ }^{40,60-}$ 62. The rTMS-locked entrainment effect in the prefrontal beta rhythm also has a causal link to memory formation. Immediate and outlasting EEG increase of beta oscillations impaired the performance of encoding memory tasks ${ }^{63}$. Overall, these results suggest that location-specific TMS applied as one strong pulse or as a train from 5 to 20 repetitive pulses at a natural frequency induces effects similar to neural entrainment.

One of the critical questions underlined in the thesis's objective is whether the effects induced by strong TMS and weak electrical stimulation and physiological entrainment, for example, by a speech or visual stimuli, can be defined as 'neural entrainment'. TMS is usually applied to elicit cortical or muscle responses and requires much stronger intensities at ca. $100 \mathrm{mV} / \mathrm{mm}$. A pulse inducing 20-40 $\mathrm{mV} / \mathrm{mm}$ does not elicit evoked potentials measured by EEG but still may induce the power changes ${ }^{59}$. In terms of neural mechanisms, strong EEG response or TMS-locked oscillations or event-related potentials (ERP) is different from induced oscillation ${ }^{64}$. Whenever the evoked oscillations reflect the dynamic response of simultaneous neural activity activation, the 
induced oscillations are mediated by modulatory mechanisms engaging massively synchronous neural assemblies. External triggers can modulate these oscillations, but they emerge by autonomous mechanisms, not directly by the stimulation. At high intensities ca. $100 \mathrm{mV} / \mathrm{mm}$, TMS induces phase-resetting effects while the ongoing oscillation activity may remain unaffected $^{65}$. In other words, TMS-locked oscillations are short-lasting and may not have cumulative effects and plasticity changes in the cortex.

Overall, biophysical and computational studies are needed to understand the complexity of neural mechanisms in response to stimulation parameters such as strength, pulse form, directionality, and the anatomical organization of neurons at the stimulation target.

\subsection{Limitation of the TMS technique}

Besides adverse effects induced by TMS, there are also technical limitations. A loud acoustic click sound accompanying each TMS pulse induces a sound pressure level of ca. $100 \mathrm{~dB}$ (for $100 \%$ of maximum stimulator output), which exceeds the safety threshold of $80 \mathrm{~dB}^{58}$. Moreover, the acoustic stimulation contributes to the neural response by indirectly triggering the auditory cortex ${ }^{66}$. At the moment, it is not possible to completely suppress the acoustic click in fully awake participants even using modern acoustic systems with external noise suppression or masking the click by white noise. Also, TMS activates peripheral motor axons resulting in the twitching of cranial muscles. The muscle activity impairs the EEG signal and cannot be removed entirely due to its physiological nature ${ }^{67}$. When stimulating the motor cortex, sensory feedback from the activated muscle results in sensorimotor areas activation that is not directly stimulated by $\mathrm{TMS}^{68,69}$. Therefore, TMS has several undesired multisensory effects that are difficult to eliminate from EEG or control by sham stimulation ${ }^{70}$. 
Many stimulation parameters, including the timing, duration of stimulation, or the induced EF strength, still reflect methodological and technological limitations rather than physiologically determined values. Most rTMS studies use the 80 to $120 \%$ of the motor threshold, which corresponds to induced EFs in the range of $14-182 \mathrm{mV} / \mathrm{mm}^{71}$. Some EF values are strong enough to evoke muscle twitch in head muscles (evoking long-lasting EEG artifacts) and in peripheral muscles when it is targeted over their cortical representation, but almost half of them $(<60$ $\mathrm{mV} / \mathrm{mm}$ ), according to the TMS-EEG studies cannot induce TMS-EEG response, therefore, do not induce action potentials ${ }^{59}$. However, no physiological recommendation exists for calibrating TMS parameters used for neural entrainment or in the cognitive domain.

Current guidelines for rTMS targeting of the non-motor regions are based on the arbitrary intensity selection reflecting safety limitations or threshold estimation procedure. Both of these approaches lack precise physiological rationalization. Moreover, including the high variability of the rTMS induced effects and high interindividual resulting EFs, it is crucial to improve the current intensity selection procedures by individualized head models and EF estimation.

\subsection{Objectives and structure of this dissertation}

The objectives of this thesis were 1) to study a neural entrainment mechanism induced by weak EF in humans; 2) to develop a new stimulation approach by adjusting the stimulation parameters (frequency and intensity) for individuals and brain regions; 3) defind the stimulation intensity range where a neural entrainment effect might occure, and 2) to study the relationships between the entrained rhythm and corticospinal excitability by examining its electrophysiological as well as neurophysiological responses. 
- In Chapter 4, a novel method for individualized rTMS stimulation intensity is introduced, called the prospective EF estimation approach. The left occipito-parietal alpha rhythm was targeted with EFs in the range of $20-50 \mathrm{mV} / \mathrm{mm}$ as a proof-of-concept that low-intensity rTMS has an immediate entrainment effect on the brain oscillations ${ }^{72}$.

- In Chapter 5, the after effect on alpha power modulation is investigated by time-frequency analysis of rTMS-EEG data ${ }^{73}$.

- In Chapter 6, low-intensity rTMS inducing $35 \mathrm{mV} / \mathrm{mm}$ in the left $\mathrm{M} 1$ is applied to target sensorimotor $\mu$-alpha rhythm and show the modulation of corticospinal excitability in $\mathrm{M} 1^{74}$. Throughout this work, the role of rTMS induced EF for neuronal entrainment was investigated. In Chapter 4, rTMS induced immediate electrophysiological effects at much weaker EF strengths than previously thought. No entrainment effect was found at intensities less than 35 $\mathrm{mV} / \mathrm{mm}$. In Chapter 5, $35 \mathrm{mV} / \mathrm{mm}$ of arrhythmic rTMS induced short-lived alpha suppression during the inter-burst intervals. In Chapter $6,35 \mathrm{mV} / \mathrm{mm}$ rhythmic rTMS synchronized $\mu$-alpha rhythm and modulated its power, which resulted in inhibited corticospinal excitability in M1. The general discussion (Chapter 7) puts this thesis's results into greater context and dissects the scientific limitations of this thesis. 


\section{Weak rTMS-induced electric fields produce neural entrainment in humans}

$\underline{\text { Elina Zmeykina }}^{1}$, Matthias Mittner ${ }^{2}$, Walter Paulus $^{1}$ and Zsolt Turi* ${ }^{1,3}$

\footnotetext{
${ }^{1}$ Department of Clinical Neurophysiology, University Medical Center Goettingen, Göttingen, Germany

${ }^{2}$ Department of Psychology, University of Tromsø, Norway 3Department of Neuroanatomy, ${ }^{3}$ Institute of Anatomy and Cell Biology, University of Freiburg, German
}

Published: Scientific reports (Publisher: Springer Nature), published on July, 2020 https://doi.org/10.1038/s41598-020-68687-8

\section{Author contributions:}

E.Z.: study design, project administration, investigation (data collection), software (prospective electric field modeling), formal analysis (EEG), visualization, data interpretation, data curation, and writing original draft.

M.M.: study design, formal statistical analysis, supervision of electric field modeling, visualization, data interpretation, and collaboration writing original draft.

W.P.: study design, funding acquisition, resources, data interpretation, and collaboration writing original draft.

Z.T.: conceptualization, study design, project administration, investigation (literature search, data collection), methodology, software (prospective and retrospective electric field modeling), formal statistical analysis, illustration, visualization, data interpretation, data curation, and writing original draft.

Copyright (C) 2020, The Author(s). This is an open access article distributed under the terms of the Creative Commons CC BY license, which permits unrestricted use, distribution, and reproduction in any medium, provided the original work is properly cited. 


\subsection{Abstract}

Repetitive transcranial magnetic stimulation (rTMS) is a potent tool for modulating endogenous oscillations in humans. The current standard method for rTMS defines the stimulation intensity based on the evoked liminal response in the visual or motor system (e.g., resting motor threshold). The key limitation of the current approach is that the magnitude of the resulting electric field remains elusive. A better characterization of the electric field strength induced by a given rTMS protocol is necessary in order to improve the understanding of the neural mechanisms of rTMS. In this study we used a novel approach, in which individualized prospective computational modeling of the induced electric field guided the choice of stimulation intensity. We consistently found that rhythmic rTMS protocols increased neural synchronization in the posterior alpha frequency band when measured simultaneously with scalp electroencephalography. We observed this effect already at electric field strengths of roughly half the lowest conventional field strength, which is $80 \%$ of the resting motor threshold. We conclude that rTMS can induce immediate electrophysiological effects at much weaker electric field strengths than previously thought. 


\subsection{Introduction}

Neurons and neural assemblies in the mammalian brain temporally synchronize their activity leading to the emergence of macroscopic network oscillations ${ }^{75}$. Network oscillations are rhythmic patterns of neural activity that are maintained in all physiologically occurring brain states ${ }^{76}$. They are crucial for intact neuropsychological functioning and are frequently disrupted in neurological or psychiatric diseases ${ }^{77}$.

However, neurons also respond to both endogenous and exogenous electric fields ${ }^{78}$. Noninvasive electrical brain stimulation (NIBS) methods, such as repetitive transcranial magnetic stimulation (rTMS), are promising techniques for modulating endogenous oscillations ${ }^{79}$. Many NIBS studies employ oscillating electric fields because it is believed that these exogenous oscillations can modulate the phase or the power of endogenous oscillations ${ }^{80}$.

The two crucial properties of rTMS-generated periodic electric fields are its frequency and its magnitude. Whereas the frequency of the electric field is clearly defined, its magnitude in the brain is defined only indirectly. Most studies choose to set the stimulation intensity using the near threshold approach. This approach defines the stimulation intensity as a percentage of the threshold intensity required to induce a liminal response in the motor or visual cortex ${ }^{81}$.

Although the near threshold approach utilizes individualized stimulation intensities, the properties of the rTMS-induced electric field, including its strength, can differ substantially within and across individuals. For example, this approach cannot account for differences in the cortical folding pattern and the cortex-scalp distance between motor and non-motor areas ${ }^{48}$. However, it is crucial to account for these known anatomical effects because the induced electric field strength plays an important role in inducing electrophysiological effects ${ }^{39}$. 
The induced electric field strength, however, remains unknown in most rTMS studies. A very limited number of retrospective estimations indicate that rTMS with conventionally used protocols induces peak electric field strengths of around $100 \mathrm{mV} / \mathrm{mm}^{82,83}$. At these high field strengths electrophysiological effects are consistently found ${ }^{40,84-86}$. However, these findings do not preclude the possibility that the effective threshold for rTMS is much lower. At least two separate lines of evidence support this assumption.

First, in vivo animal studies have shown weak, but reliable, electrophysiological effects already at field strengths in the range of 0.3 and $1 \mathrm{mV} / \mathrm{mm}^{87,88}$. This electric field range can temporally bias spike timing or might even entrain network oscillations ${ }^{39,89}$. Second, it has been found that even the weak electric fields induced by sham rTMS (ca. $5 \mathrm{mV} / \mathrm{mm} ; 15$-fold weaker than active rTMS) can induce short-lasting electrophysiological aftereffects in humans ${ }^{82}$.

Based on this converging evidence, we hypothesized that we should be able to observe immediate electrophysiological effects using electric fields between 20 and $50 \mathrm{mV} / \mathrm{mm}$. The 20 $\mathrm{mV} / \mathrm{mm}$ electric field corresponds to the stimulator's lower limit of producing real rTMS (detailed in Supplemental Method, Validation measurement). This electric field range covers a "middle ground" between electrical brain stimulations, such as transcranial alternating current stimulation (tACS) and rTMS. On the one hand, the field strength is above the range of $0.3-1 \mathrm{mV} / \mathrm{mm}$, and therefore stronger than the electric field used by conventional $\mathrm{tACS}^{88}$. On the other hand, these values are several orders of magnitude weaker than those used in the near threshold approach, where the electric fields are around $100 \mathrm{mV} / \mathrm{mm}^{82}$.

To test our hypothesis, we took an alternative approach to the conventional near threshold method. We refer to it as the prospective electric field estimation approach (for an overview, see Figure $3 \mathrm{~A}$ and Table 3 ). The decisive feature of our approach is that prospective computational 
modeling of the magnitudes of the induced electric fields guided the choice of stimulation intensity at the individual subject level. Moreover, we estimated individual peak frequencies of posterior alpha oscillations to fine-tune the stimulation frequency. Finally, real-time neuronavigation ensured accurate and consistent targeting across the sessions.

By using this approach, our focus was on inducing immediate electrophysiological effects on posterior alpha oscillations in humans. The reasons for focusing on posterior alpha oscillation are that it has a high signal-to-noise ratio in resting state measurements, and that its peak frequency has a low intra-subject variability ${ }^{17}$. To characterize the immediate electrophysiological effects of rTMS, we calculated the phase locking value (PLV) of the simultaneously recorded scalp electroencephalography (EEG) ${ }^{86,90,91}$. The PLV captures the extent of neural synchronization by measuring the amount of phase alignment between the rTMS and network oscillations assessed by EEG. Our novel individualized intensity selection method for rTMS with prospective electric field modeling shows that neural entrainment occurs at lower than expected field strengths. 


\subsection{Results}

\subsubsection{Study overview}

The present study consisted of a main and a control experiment (for an overview see Figure 3). In the main experiment (Figure 3B, top), we employed a single-blind, randomized, cross-over study design, using an active control rTMS condition within participants and sessions. The participants $(\mathrm{n}=16)$ took part in five experimental appointments including one neuroimaging session, one session for motor threshold hunting, and three rTMS-EEG sessions.

In the rTMS-EEG sessions, the participants received rTMS stimulation at intensities prospectively estimated to induce EFs of three different magnitudes: 20,35 and $50 \mathrm{mv} / \mathrm{mm}$. These values correspond to the group-level mean $( \pm \mathrm{SD})$ of $9.5 \pm 1.1 \%, 16.8 \pm 2 \%$ and $23.9 \pm 2.5 \%$ of the maximum stimulator output, respectively. We obtained peak magnitudes of the absolute electric field extracted from the gray matter compartment. The center of the coil was placed over the PO3 electrode. We applied each dose on individual sessions separate by at least 48h. During each rTMSEEG session, we applied rhythmic (main) and arrhythmic (active control) rTMS protocols. All stimulation parameters except the rhythmicity were identical in both protocols.

In the rhythmic protocol, we set the stimulation frequency to the individual alpha frequency following the Arnold tongue model. This model assumes that neural entrainment is most effective when the stimulation frequency matches the endogenous frequency ${ }^{40,92}$. In the arrhythmic protocol, the same number of pulses as in the rhythmic protocol was presented but the inter-pulse interval was randomized in order to remove frequency-specific stimulation effects ${ }^{40,86}$. Apart from the rhythmicity, all stimulation parameters (stimulation intensity, location, number of TMS pulses) were identical in both protocols. The two protocols would probably produce closely matched 
acoustic and somatic sensations. We applied the stimulation with the participants at rest, and we instructed them to keep their eyes open.

In order to control for potential effects induced by the acoustic by-products of the rTMS device, we performed an additional control experiment on a separate group of participants $(\mathrm{n}=16$; Figure 3B, bottom). We chose a commonly used sham procedure, in which we tilted the stimulation coil by $90^{\circ}{ }^{93}$. This sham protocol emulates the rTMS-induced click sounds that might induce a spurious increase in occipital alpha synchronization ${ }^{94}$ while minimizing any direct effects of the stimulation. In the control experiment, the participants received a single rhythmic rTMS session applied at a fixed stimulation intensity of $29 \%$ of the maximum device output. This value corresponds to the highest stimulation intensity applied in the main experiment. We chose this value in order to maximize the noise level of the sham rTMS, which increases with stimulation intensity. The stimulation frequency was set to the individual alpha frequency. Apart from the stimulation intensity, all the remaining parameters were kept constant as in the main experiment (detailed in section 2.4.10). 
A
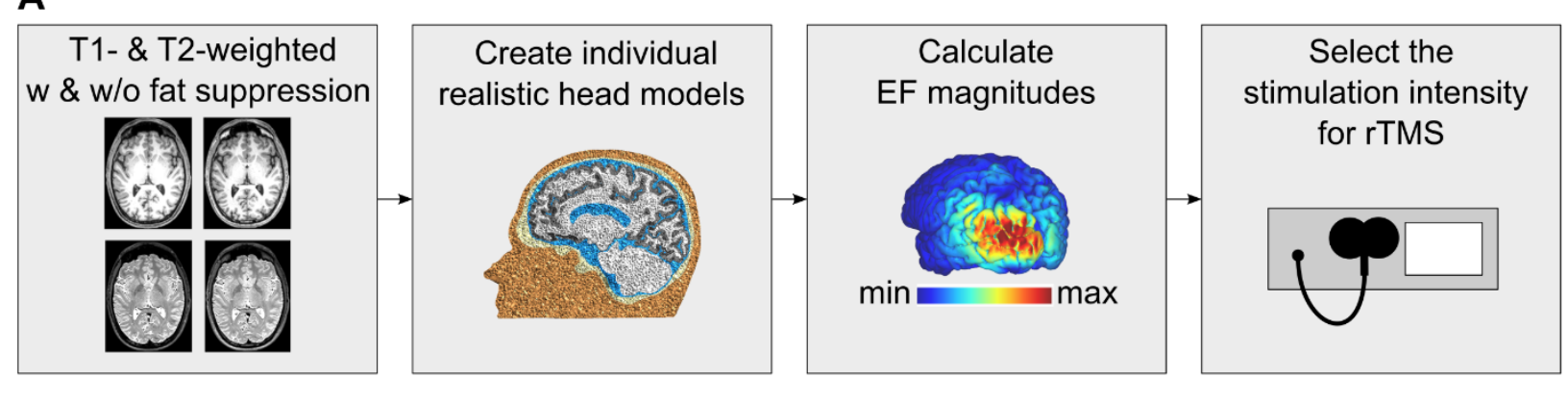

B

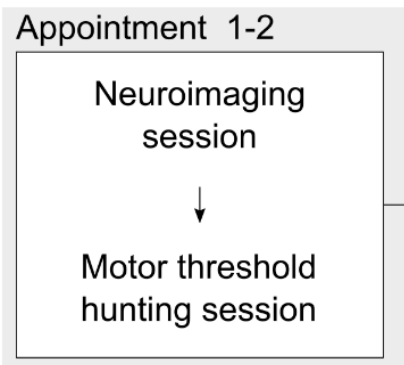

\section{Appointment 3-5}

Main experiment $(n=16)$

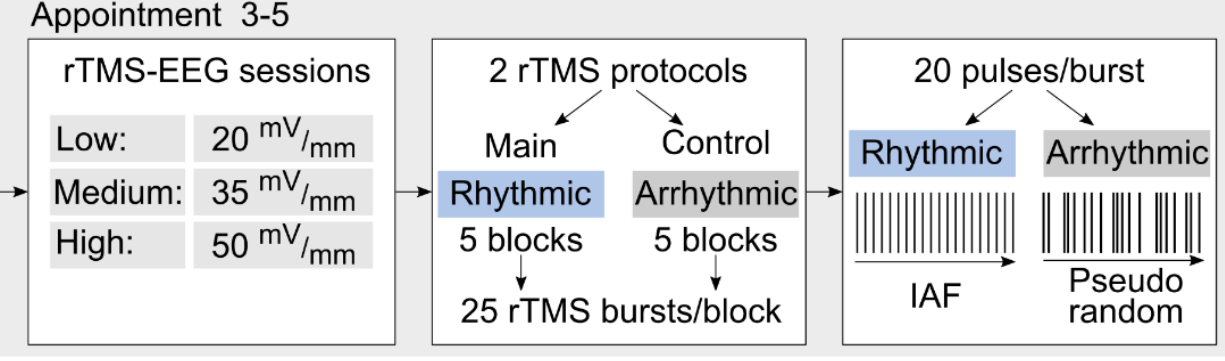

Control experiment $(n=16)$

Appointment 1

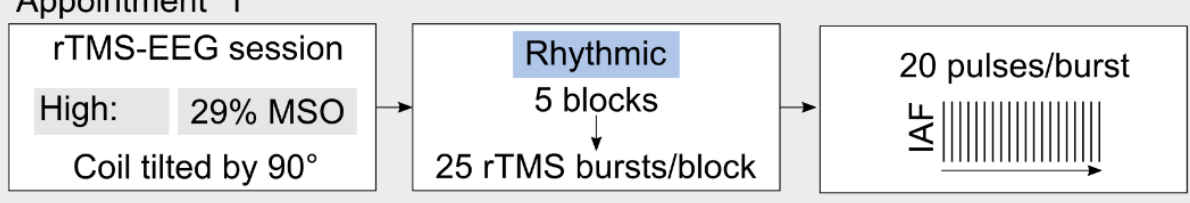

Figure 3. Study overview. (A) Schematic of the prospective electric field estimation approach. (B) Appointments and stimulation protocols in the main (top) and control (bottom) experiments. Abbreviations: EF - electric field; MSO\% - percentage of maximum stimulator output; IAF individual alpha frequency.

\subsubsection{Rhythmic rTMS synchronizes ongoing posterior}

\section{alpha rhythms}

We performed the following analysis to characterize the immediate electrophysiological effects of rTMS in the main and control experiment. In the preprocessing stage of the data analysis, we removed the TMS-induced artifacts from the EEG data. We used the same preprocessing 
algorithm for all stimulation conditions. A detailed description is given in section 2.4.11. In brief, we eliminated ringing artifacts by removing data from $4 \mathrm{~ms}$ before to $9 \mathrm{~ms}$ after the TMS pulse. Next, we ran an independent component analysis (ICA) to remove decay artifacts. We then interpolated the time interval around each TMS pulse.

Further, we used a semi-automatic algorithm adapted from the open-source toolbox ARTIST to eliminate further artifacts ${ }^{95}$. We defined trials or channels as contaminated with artifacts if their power exceeded 1.5 times the interquartile range. If the artifacts affected fewer than $20 \%$ of all channels, we interpolated signals from the non-contaminated channels, or otherwise removed the entire trial. Moreover, we removed channels with a large standard deviation (STD $>30 \mu \mathrm{V})$. We also estimated the correlation coefficient of the signal of each channel with its neighbors and removed and interpolated those channels with a low correlation coefficient $(<0.4)$. Finally, we removed blinks, saccades and other eye-related movements by ICA. Figure 4 shows the raw data before and after the artifact removal process from three example participants. 
S1, $26 \% \mathrm{MSO}$

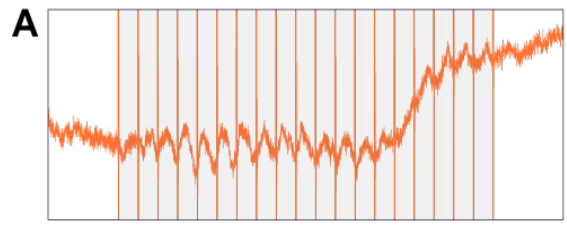

B

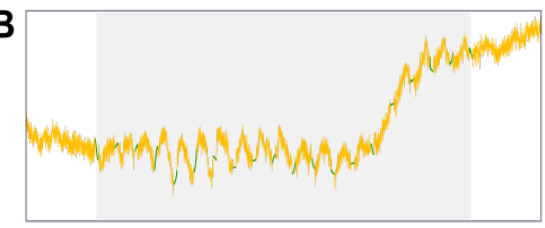

C

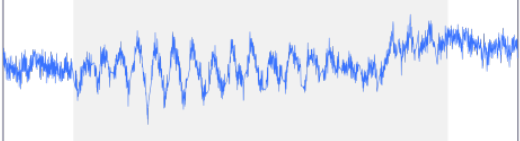

S2, $29 \% \mathrm{MSO}$
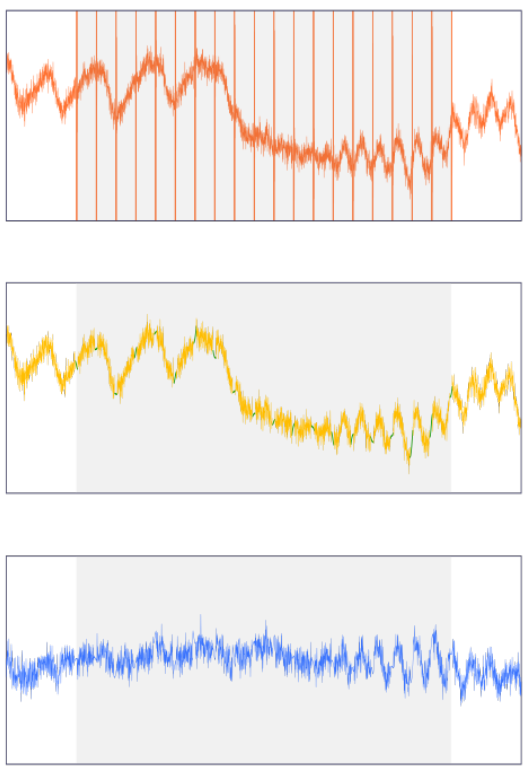

S3, $29 \% \mathrm{MSO}$
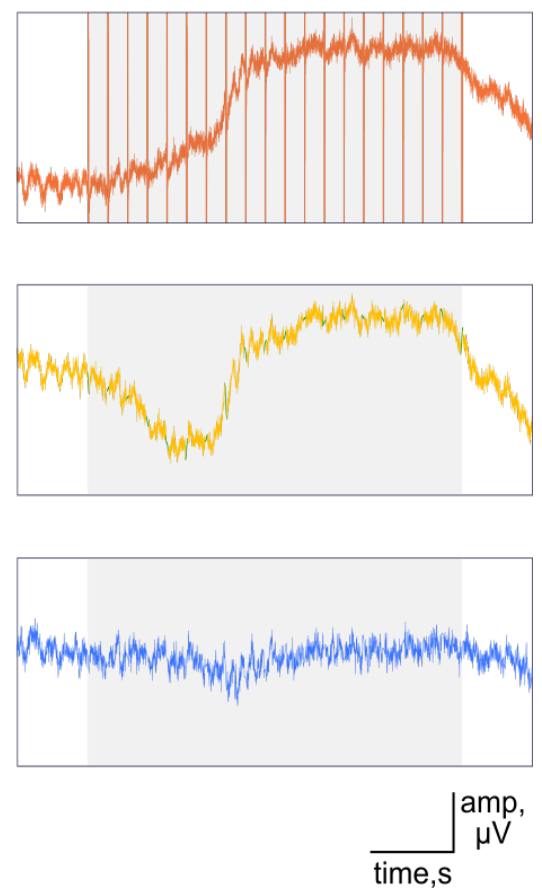

Figure 4. Artifact removal for three example datasets. Onset and offset of rTMS are shown as grey rectangle, individual pulses are shown by red vertical lines. (A) Raw data from the POz channel. (B) The removal and interpolation (green) of ringing artifacts. (C) Data at the end of preprocessing.

Next, we measured the amount of synchronization in the scalp EEG signal. For this, we first applied wavelet decomposition of the EEG signal and extracted the phase information from the imaginary component of the Fourier coefficient (see section 2.4.11). We then simulated sinusoidal waves based on the individual stimulation frequencies and phase-aligned them to the offset of the TMS pulses. We computed the PLV between the EEG signal and the sinusoidal wave for each rTMS intensity condition and rTMS protocol, respectively.

Based on the Arnold tongue model of neural entrainment ${ }^{92}$, we expected that the intervention would synchronize the ongoing endogenous brain rhythm to the rhythmic rTMS. We also expected that the arrhythmic or sham protocols would not affect the amount of synchronization. To test this 
hypothesis, we first determined how rTMS affected the amount of neural synchronization as measured by the PLV in the individual alpha frequency relative to baseline. We defined the baseline as the time window $500 \mathrm{~ms}$ before rTMS onset. We normalized to baseline with the relative-change method: A baseline normalized value of 1 indicates no change in the PLV, a value of 0.5 shows a $50 \%$ decrease and value of +1.5 corresponds to a $50 \%$ increase. We found that rhythmic rTMS increased the PLV across all rTMS intensities (Figure 5A, top), while arrhythmic rTMS had no such effect (Figure 5A, bottom). As expected, with rhythmic rTMS we observed the greatest PLV increase over the posterior electrodes (Figure 5A, top). This was not the case for the arrhythmic or sham protocols.

Because we stimulated the posterior parietal-occipital cortex, we studied the time course of PLV change in the posterior electrodes for frequencies between 5 and $30 \mathrm{~Hz}$ (Figure 5B). We aligned the data to the offset of the rTMS burst, which is indicated by the vertical line at $(0 \mathrm{~s})$ on the time axis. Because we delivered rTMS at the individual alpha frequency and kept the number of pulses constant, the duration and hence the onset time of the rTMS bursts varied (e.g., $8 \mathrm{~Hz}$ : $2.5 \mathrm{~s} ; 12 \mathrm{~Hz}: 1.67 \mathrm{~s})$. In the rhythmic rTMS protocol, we found that PLV increased after rTMS onset and returned to the baseline after rTMS offset (Figure 5B, top). The increase in PLV was strongest in the ongoing alpha frequency band and its harmonics in the beta frequency range. For the arrhythmic protocols, we found no change in the PLVs (Figure 5B, bottom). For the sham rTMS, we observed an initial increase in the theta and alpha frequency bands (Figure 5B, right) but no sustained increase in PLV throughout stimulation. 
A

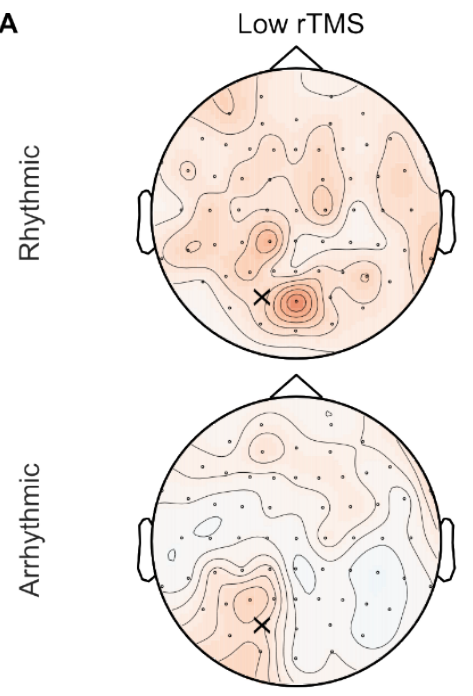

B
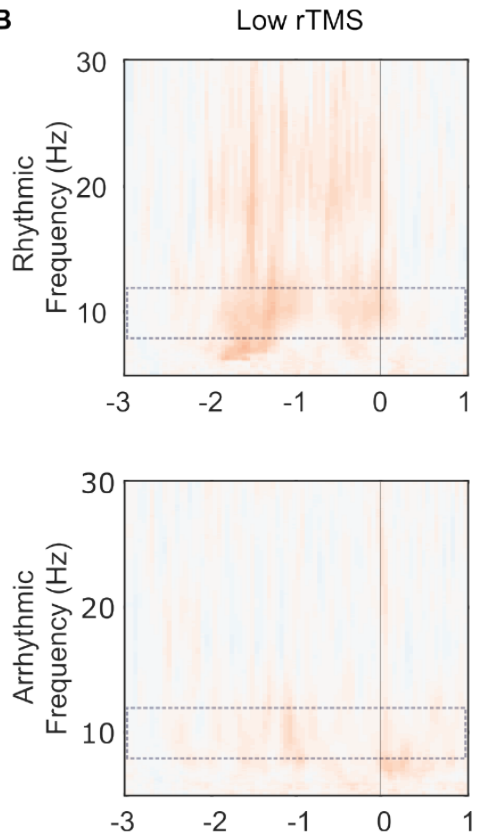
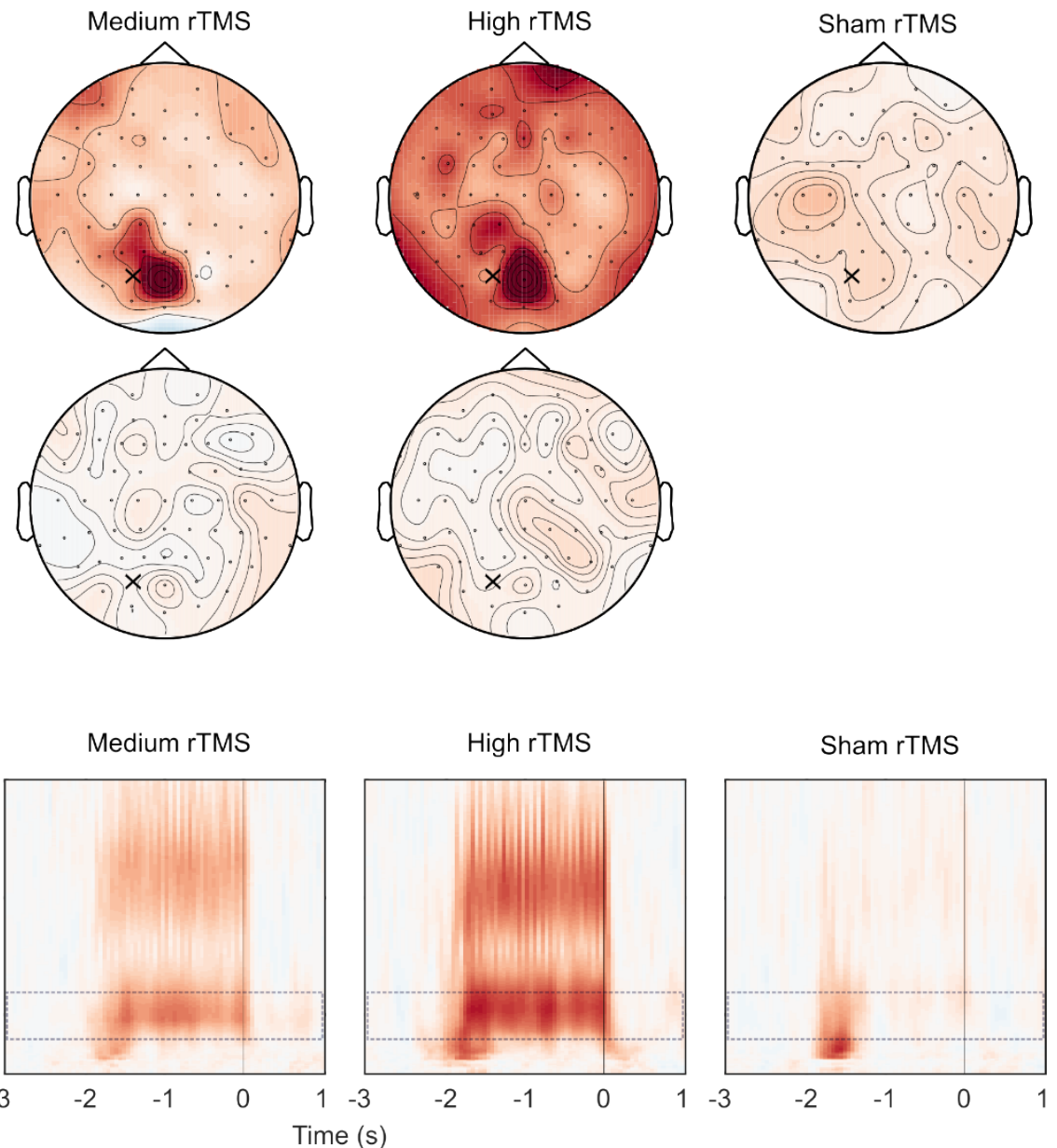

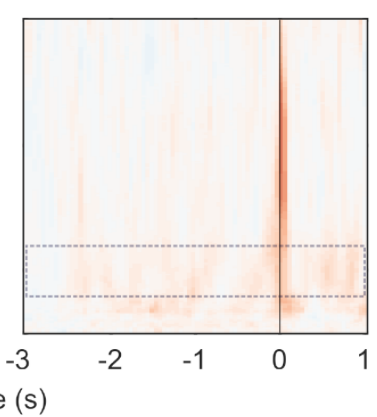

Phase locking value, relative change

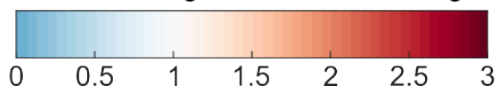

Figure 5. Rhythmic rTMS synchronized ongoing posterior alpha rhythms indicated by increased phase locking values. (A) The degree of synchronization at the individual alpha frequencies was most pronounced in the posterior electrodes for the real rhythmic (top row) but not for the sham rhythmic (top, right) or arrhythmic rTMS (bottom row). We applied rTMS over the PO3 electrode 
(location marked with the cross). (B) Rhythmic rTMS (top row) synchronized ongoing posterior alpha rhythms and its first harmonics in the posterior electrodes. Compared to baseline, sham rTMS induced a short-lasting increase in the PLVS in the theta and alpha frequency band. The alpha band is shown with a dashed rectangle. Color represents the changes of phase locking value relative to baseline from -3 to $-2.5 \mathrm{~s}$ prior to rTMS offset. Timepoint $t=0$ corresponds to the last pulse of all rTMS bursts in panel B.

We performed two control analyses to ensure that the PLVs change in the main rhythmic rTMS condition was not due to artifacts or induced by our preprocessing pipeline (detailed in section 2.4.11). First, a spurious increase in PLVs can potentially arise if ringing and decay artifacts are only incompletely removed. We therefore performed a control measurement on a piece of meat using the identical stimulation, measurement and analysis parameters as in the main experiment. This analysis confirmed that our preprocessing pipeline removed these artifacts, as we detected no increase in PLV (Figure 6, A).

Second, a potential bias could arise from the periodical exclusion of intervals corresponding to the rTMS-induced ringing artifacts and their interpolation. We tested for this bias by performing a control analysis on artifact-free resting state EEG data that had been recorded from the 16 participants before each rTMS-EEG session in the main experiment (see section 3.4.11). This control analysis confirmed that our analysis pipeline did not increase the PLVs under the main rhythmic rTMS condition (Figure 6, B). 
$\begin{array}{ll}\text { A. rTMS phantom, } 29 \% \text { MSO } & \text { B. rTMS simulated }\end{array}$

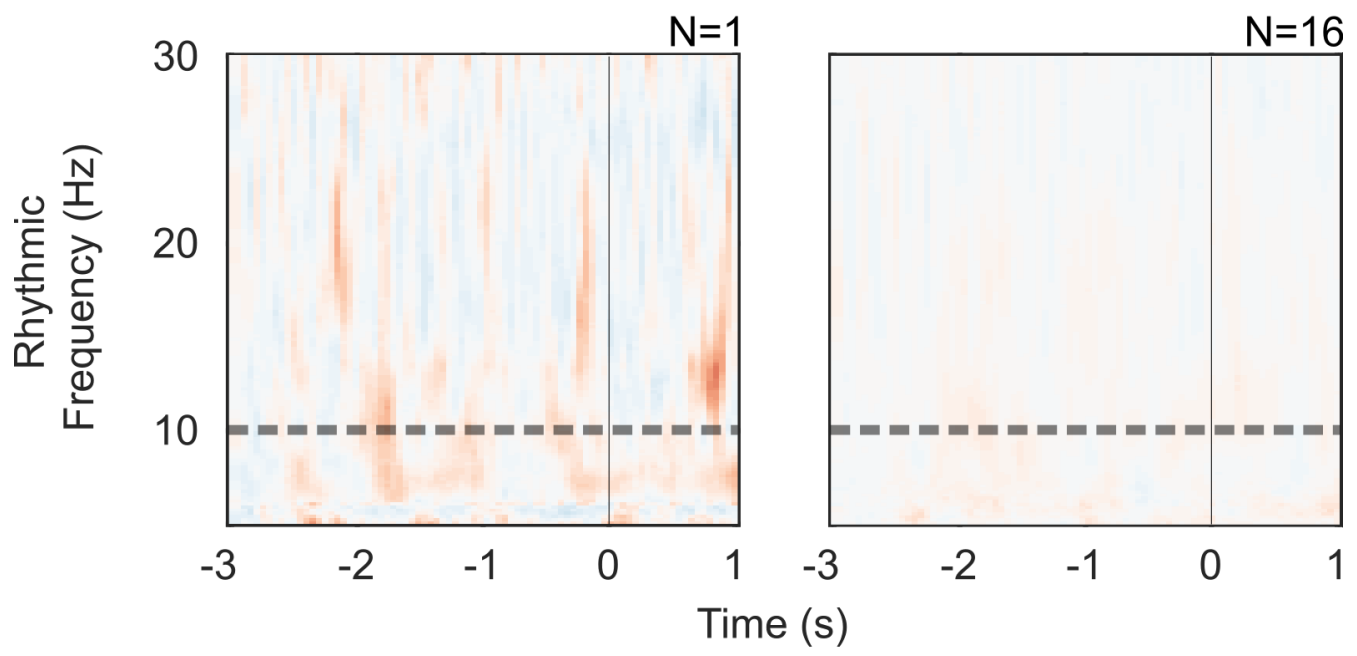

Phase locking value, relative change

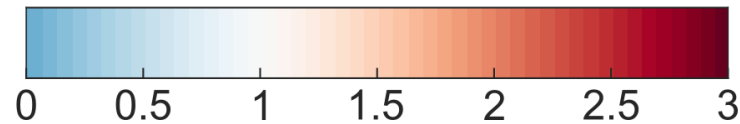

Figure 6. Preprocessing pipeline does not artificially increase the degree of neural synchronization. (A) Control measurement on piece of meat. (B) Control analysis on artifacts free resting state EEG data.

\subsection{3. $\mathrm{rTMS}$ induces rapid and sustained increase in the ongoing posterior alpha synchronization}

Next, we focused on the PLV time course at the individual alpha frequency (Figure 7). In the rhythmic rTMS condition, the PLV increased rapidly after the onset of rTMS and returned to baseline after stimulation offset (Figure 7A). The offset-locked data analysis introduced variability in the initial part of the time course of the PLV, due to varying onsets of the stimulation for each participant. Thus, we also computed PLVs locked to each TMS pulse (Figure 7B). This analysis 
was only conducted on the rhythmic rTMS protocols since they are characterized by constant interpulse intervals.

The initial pattern of PLV increase was similar for all rhythmic rTMS intensity conditions. However, the time course of the PLV change was slightly different for the three main rTMS intensity conditions. In the low rTMS intensity condition, we found that the mean PLVs returned to the baseline after an initial increase (Figure 7B, left). In the middle and high rTMS intensity conditions, the induced increase in the PLV was stable over the time course of the stimulation (Figure 7B, middle and right) and the level on which the PLV plateaued was higher for the highest intensity condition. We observed an initial increase in the PLVs also in the sham condition. However, this initial increase shortly returned to the baseline value after the first five pulses and did not show the sustained pattern observed in the two active stimulation conditions.

We then compared the PLVs during the stimulation period of the rhythmic, arrhythmic and sham rTMS protocols using independent non-parametric cluster-based permutation tests at each rTMS intensity condition. The PLVs in the rhythmic rTMS were significantly higher than in the arrhythmic rTMS at medium and high intensities (both $\mathrm{p}<0.001$ ) but just missed significance at the low rTMS intensity $(\mathrm{p}=0.054)$. Sham rTMS resulted in significantly higher PLVs compared to the rhythmic low rTMS $(\mathrm{p}=0.011)$ and arrhythmic rTMS at all intensities $(\mathrm{p}<0.001)$. Real rhythmic rTMS applied at medium and high intensities resulted in significantly higher PLVs than the sham rTMS $(\mathrm{p}=0.042$ and $\mathrm{p}=0.003)$. 

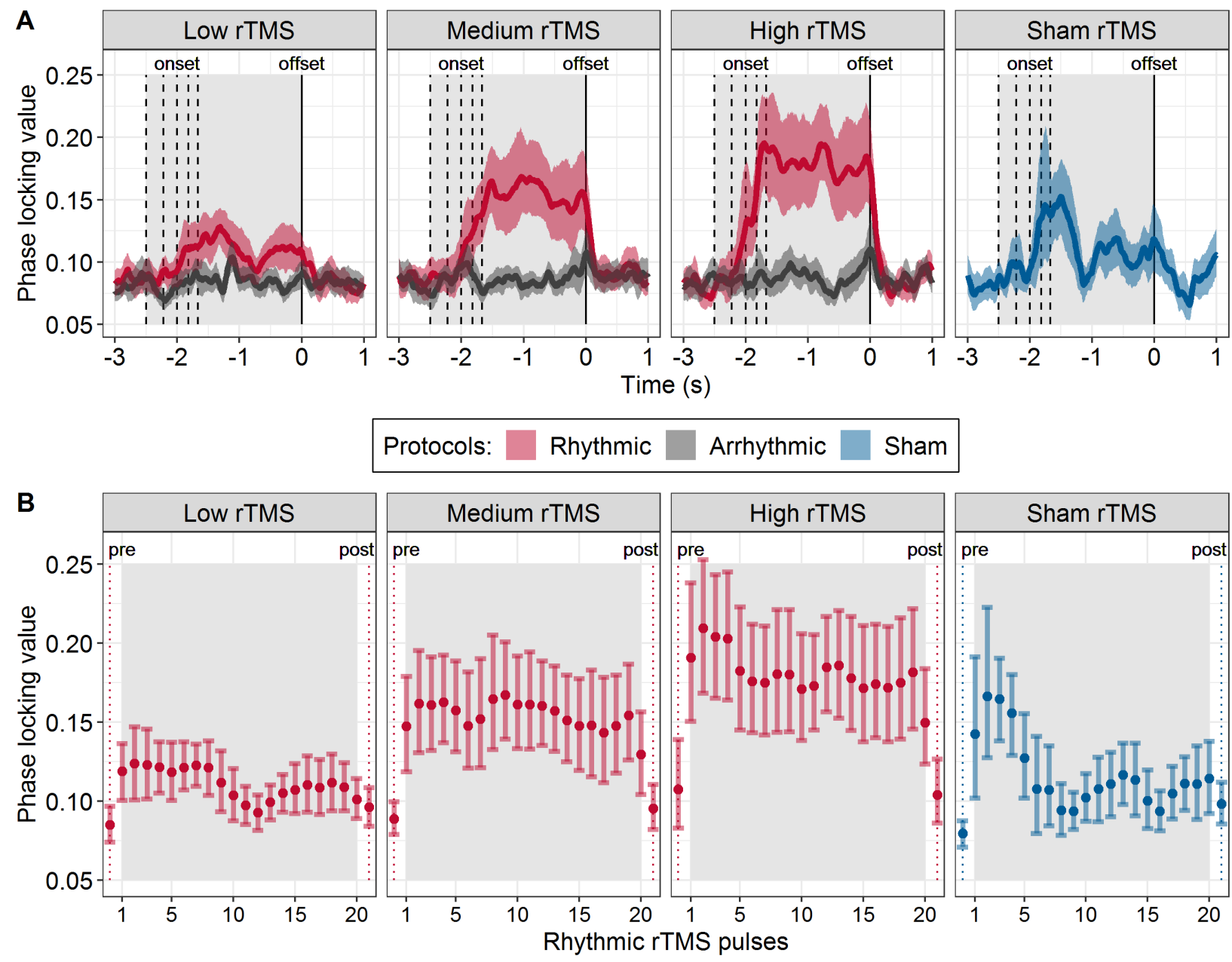

Figure 7. Increased and sustained neural synchronization during rhythmic but not during arrhythmic or sham rTMS. (A) The time course of the phase locking value calculated for individual alpha frequencies is shown for the parietal channels. Vertical dashed lines correspond to the stimulation onset at individual alpha frequencies (between 8-12 Hz; from left to right). The vertical solid line shows the rTMS burst offset ( $0 \mathrm{~s})$. Phase locking values are aligned to the offset of the rTMS burst. Solid lines represent mean phase locking values, the shaded areas show the 95\% confidence intervals. (B) Phase locking values for the real and sham rhythmic rTMS conditions, aligned to each of the 20 rTMS pulses. The dots represent means, and the error bars 95\% confidence intervals. Dotted lines show phase locking values before the first rTMS pulse (pre) and after the last rTMS pulse (post). The light gray rectangle highlights the time window during which rTMS was applied. 


\subsubsection{Mean and median electric field strengths are in the effective range}

We characterized the mean and median electric field (EF) strengths across a number of posterior regions of interest (ROIs) as shown in Figure 8 and the peak electric field values shown in Figure 10. We selected regions in the parietal and occipital lobes that were in the vicinity of the stimulation target. As previous in vivo animal studies have demonstrated immediate

electrophysiological effects at EF strengths of ca. $1^{\mathrm{mV}} / \mathrm{mm}$, we used this value as the reference to which we compared the rTMS-produced EF values observed in the present study.

For both the absolute EF (Figure 8A) and for the normal component (Figure 8B), the mean and median EF strengths were higher than the $1^{\mathrm{mV}} / \mathrm{mm}$ reference value (and weaker than $-1^{\mathrm{mV}} / \mathrm{mm}$ ) in all rTMS conditions in the target hemisphere. In the right hemisphere, most ROIs experienced an EF stronger than $1 \mathrm{mV} / \mathrm{mm}$ only in the medium and high intensity conditions. Similarly, the peak EF values (both the absolute and the normal component) were higher than the $1 \mathrm{mV} / \mathrm{mm}$ reference value in both hemispheres (see Figure 10). 

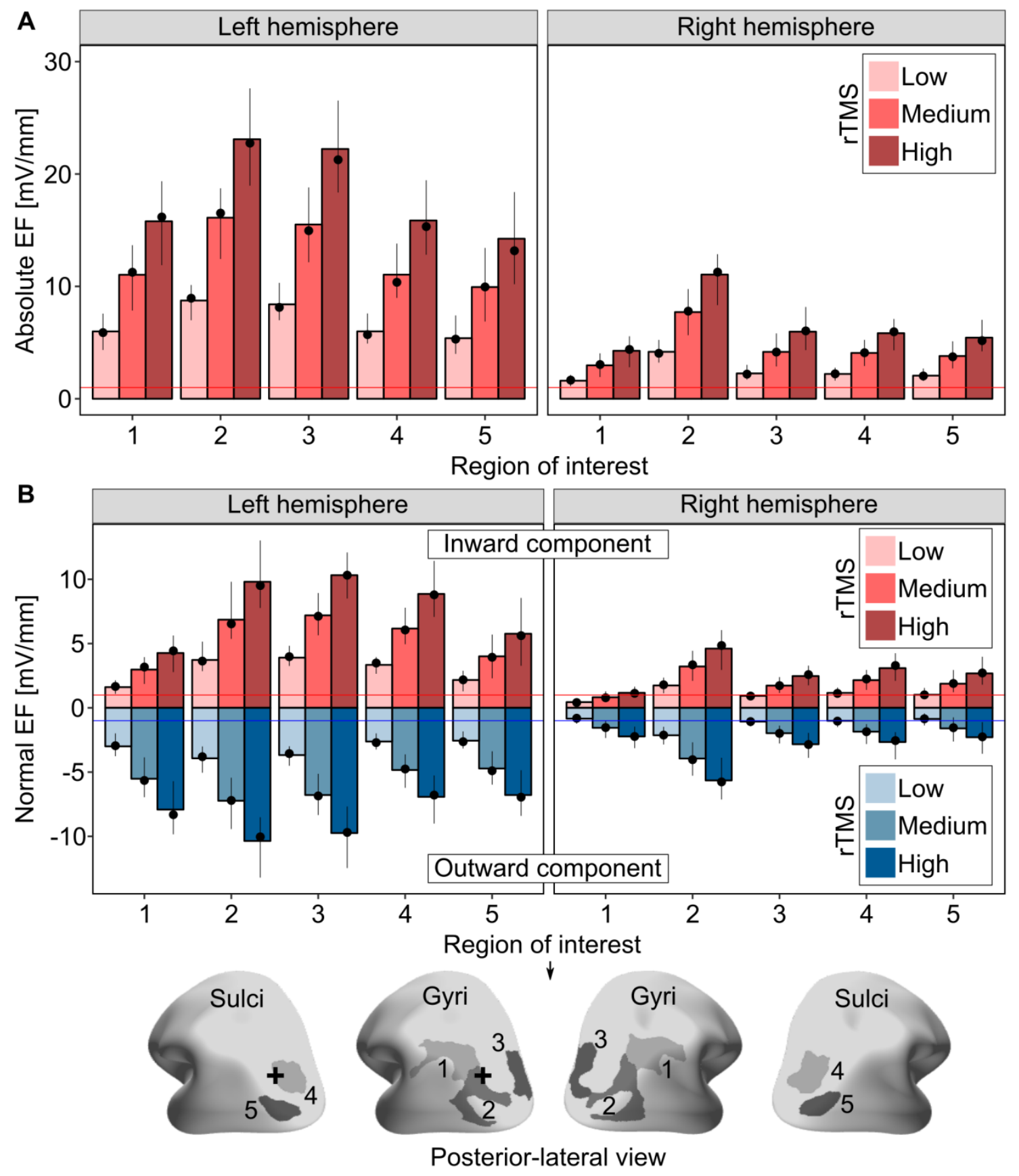

Figure 8. Group-level mean and median values of the electric field for parietal and occipital ROIs. Bar plots show the mean, dot plots show the median electric field values of the (A) absolute EF and $(B)$ its normal component. Range plots correspond to the $2.5^{\text {th }}$ and the $97.5^{\text {th }}$ percentiles of the electric field values, respectively. Red and blue horizontal lines correspond to the $1^{\mathrm{mV} / \mathrm{mm}}$ and $I^{m V} / m m$ EF strengths. A black plus sign indicates the center of the TMS coil. Range of interest: 1 - 
Angular gyrus, 2 - Superior occipital gyrus, 3 - Middle occipital gyrus, 4 - Superior occipital sulcus and transverse occipital sulcus, 5 - Middle occipital sulcus and lunate sulcus.

\subsubsection{Immediate effects in the range of 30 to $42 \%$ of the resting motor threshold}

In order to make our results more interpretable, we expressed the EF strengths in terms of maximum stimulator output (Figure 9A), and resting motor threshold percentages (Figure 9C). We used the motor threshold approach because it is the most frequently used approach in the literature (Table S1). We also characterized the resting motor threshold as the percentage of the maximum stimulator output (Figure 9B). Intensities in the range of ca. 30-42\% of the resting motor threshold were already capable of inducing immediate electrophysiological network effects in humans. The low stimulation intensities were well tolerated by the participants, who reported no phosphenes, and only a minor amount of somatosensory discomfort during rTMS (see Figure 12), which is a major concern when using higher stimulation intensities ${ }^{\text {e.g., }} 96$.
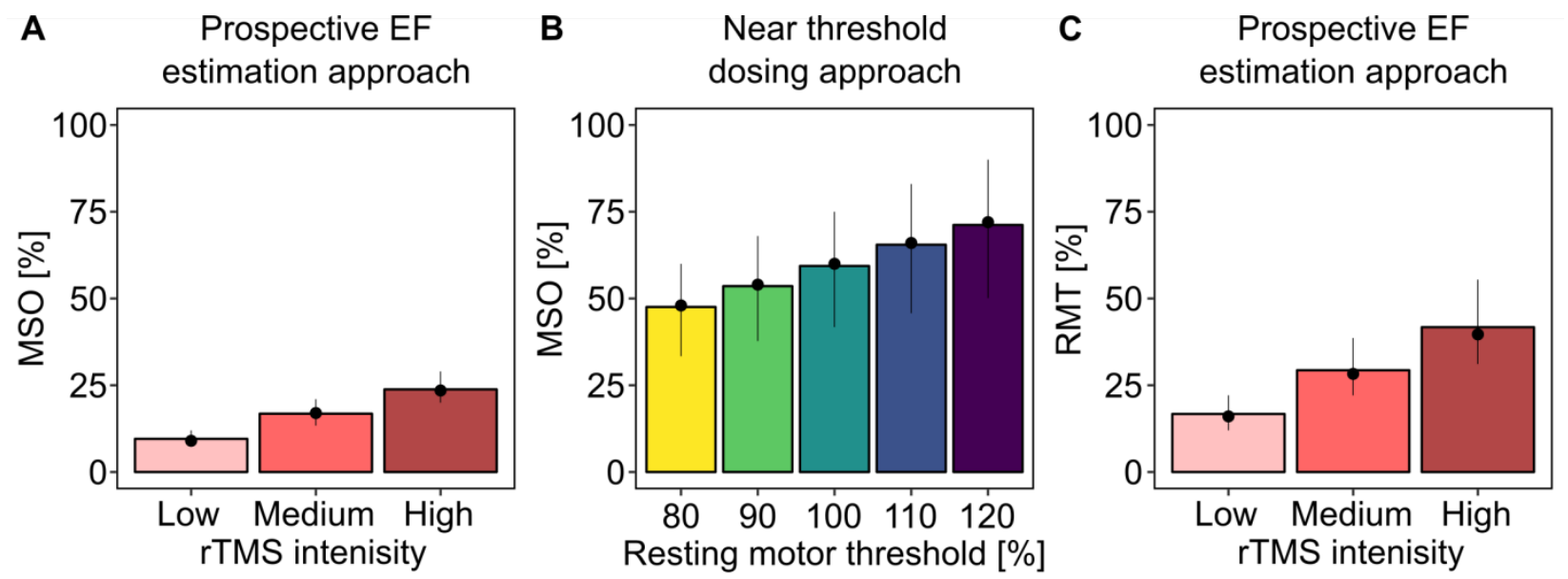
Figure 9. Our prospectively determined rTMS settings fell in the range of 17-42\% of the resting motor threshold. (A) The resulting electric fields and (B) the resting motor thresholds between 80 and $120 \%$ expressed in percentages of the maximum stimulator output. (C) Resulting electric fields expressed in percent of the $100 \%$ resting motor threshold. Bar plots show means, and dots the median values. Range plots correspond to the $2.5^{\text {th }}$ and the $97.5^{\text {th }}$ percentiles, respectively. Abbreviations: EF - electric field; MSO - maximum stimulator output; RMT - resting motor threshold.

\subsection{Discussion}

Using prospectively individualized intensities for rTMS we showed that electric fields half the magnitude of conventionally applied fields (see Figure 10, B and D) already induced immediate electrophysiological effects in humans. In the rhythmic rTMS protocols, the amount of neural synchronization increased rapidly after rTMS onset and returned to the baseline after rTMS offset. The field strength played a modulating role in inducing the effects. In the low rTMS intensity condition, the immediate effects were not statistically different from sham rTMS. In the medium and high rTMS intensity conditions, the effect was statistically significant and appeared to be stable over the time course of the rTMS burst. This was not the case for the sham rTMS, which only produced an initial, short-lived effect. Furthermore, we observed different PLV topographies between the real and the sham rTMS protocols (Figure 5A). In the real protocols, the PLV increase appeared over the middle parietal and occipital electrodes. In the sham protocol on the other hand, we observed the highest PLVs over the left temporal electrodes. In the arrhythmic protocol, which served as an active control, rTMS did not affect the amount of neural synchronization. We conclude that rhythmic rTMS applied at peak absolute electric fields from 35 to $50 \mathrm{mv} / \mathrm{mm}$ can induce immediate electrophysiological effects in humans. 


\subsubsection{Comparing our results with previous}

\section{electrophysiological findings}

In the rhythmic rTMS protocol, the observed pattern in the time course of the neural synchronization reproduced many aspects of previous tACS and rTMS findings ${ }^{40,88,89,93}$. Animal studies assessing spike timing activity revealed an immediate increase in the degree of neural synchronization during tACS ${ }^{88,89}$. Similar to our own findings, this increased activity returned to baseline immediately after the end of stimulation ${ }^{88,89}$.

A previous study assessed the effect of rhythmic rTMS at the individual peak beta frequency

93. The participants received rTMS with a conventional stimulation intensity $(90 \%$ of active motor threshold) while at rest ${ }^{93}$. The authors found that rhythmic rTMS increased the degree of neural synchronization compared to sham stimulation (coil tilted by $90^{\circ}$ ) or to control frequencies ${ }^{93}$.

On the other hand, our findings are slightly different than those of another rTMS study ${ }^{40}$, in which the stimulation was applied at the individual peak alpha frequency over the parietal cortex with the conventional intensity of $100 \%$ phosphene threshold ${ }^{40}$. These authors found that after an initial rapid increase, the degree of neural synchronization gradually decreased during the second part of the rTMS burst ${ }^{40}$. Surprisingly, the arrhythmic protocol in their study also initially increased the degree of neural synchronization ${ }^{40}$. This is contrary to our findings as we failed to find any changes in neural synchronization in our arrhythmic protocol.

One possible explanation for these divergent findings might be that even single pulse highintensity TMS can induce alpha frequency oscillations in the occipital cortex ${ }^{59}$. These studies used high rTMS intensities of approx. $100 \mathrm{mv} / \mathrm{mm}$, which could have induced the observed degree of neural synchronization with the first rTMS pulse in the arrhythmic protocol ${ }^{40}$. Careful analysis of our own data revealed a similar pattern, especially with the higher intensity conditions. There seems 
to be a slight increase in the degree of synchronization after the last rTMS pulse in the arrhythmic protocol (see Figure 5B and 7A). Note that our analysis was locked to the offset rather than to the onset of the rTMS burst. However, maintaining the neural synchronization over time requires rhythmically delivered rTMS pulses.

\subsubsection{Relation of electric field strengths to previous tACS and rTMS studies}

The electric field magnitudes in the medium and high rTMS conditions are several-fold stronger than the already effective magnitudes observed in animal studies ${ }^{88,89}$. Furthermore, the employed intensities were several-fold weaker than those applied in previous rTMS studies ${ }^{40,86}$ and therefore cover a "middle ground" between the two techniques that may be particularly suited to study immediate electrophysiological effects.

However, it is challenging to directly compare the exact electric field values in the literature, because systematic studies are lacking. Instead, studies are divided on species, stimulation frequencies, stimulation methods and the state of the receiving brain ${ }^{88,89,97}$. For example, the differences in the electric fields generated by tACS and rTMS make a direct comparison difficult. One important difference relates to the cycle/pulse duration. For tACS, depending on its frequency, a single cycle is in the range of up to several hundreds of ms. On the other hand, the width of a biphasic rTMS pulse lasts for only several hundred of $\mu$ s. This difference is crucial because pulse duration is an important temporal characteristic of the induced field. Keeping the magnitude and the pulse/waveform constant, longer pulses deliver a higher total charge than shorter pulses ${ }^{98}$. Another important difference between tACS and rTMS are the spatial components (e.g., normal, 
tangential) of the induced electric field, with TMS inducing stronger tangential components than $\operatorname{tACS}^{99}$.

\subsubsection{Towards a better understanding of the neural mechanisms of rTMS}

For several decades, the near threshold approach has been the most commonly used intensity selection method for rTMS ${ }^{81,100}$. This approach is uncomplicated and offers individualized stimulation intensities. It selects the stimulation intensity based on evoking a liminal response in the visual or motor system ${ }^{101}$ and therefore offers a rough approach to taking individual variation into account.

Externally inducing liminal responses, however, requires a strong electric field, and the functional effect of such an electric field is mostly interferential in nature. The prime example of such functional interference is rTMS-induced speech arrest ${ }^{102}$. When rTMS is applied over the left posterior-inferior frontal region, i.e., the facial motor cortex, it can block the ability to speak ${ }^{102}$.

Yet, the purpose of rTMS in neuroscience and clinical applications is primarily a targeted enhancement of function rather than interference but see 103 . From a neuroscience point of view, achieving functional enhancement is more challenging than producing interference. This is because the former requires a better understanding of the involved neural mechanisms. But what neural mechanisms does rTMS activate when applied at 80 or $120 \%$ of the resting motor threshold? One crucial limitation of the near threshold method is that it offers no clear physiological justification for the choice of stimulation intensity; the magnitude of the resulting electric field as well as the underlying neural mechanism of rTMS remain unknown. 
Recent developments in computational modeling may help to overcome this limitation as exemplified in the present study. Anatomically realistic head modeling and electric field calculations have the merit of linking the stimulation intensities to the resulting electric field strengths ${ }^{104-106}$. This can facilitate the mapping between electric field strength, neural mechanisms and functional effects ${ }^{107}$. A better understanding of the neural mechanisms of rTMS benefits both neuroscience research and the clinical application of the method ${ }^{108,109}$. For example, many such applications are aimed at restoring altered oscillatory activity, e.g. in schizophrenia, stroke or epilepsy ${ }^{110}$. One can achieve this by the targeted external control of altered oscillations though specific neural mechanisms, e.g. neural entrainment.

However, even the state-of-the-art head-modeling approaches are only an approximation of the true individual anatomy. Known sources of inaccuracy include segmentation errors ${ }^{111}$, a limited number of tissue types ${ }^{112}$, and the use of standard, but possibly incorrect, conductivity values 113,114. Despite these limitations, we remain convinced that computational models are invaluable for prospectively adjusting the stimulation intensities for rTMS. They can provide new insights into how an electric field generated from outside the head by rTMS can produce immediate electrophysiological effects in the human brain. We conclude that individualized prospective electric field strength calculation is an essential approach to better understand the neural mechanisms of rTMS.

\subsubsection{Future directions}

The precision of determining the anatomical target possibly further affects the immediate electrophysiological effects. We expect that individualizing the stimulation target based on anatomy, e.g., targeting the inferior parietal sulcus, or EEG source estimation-based localization 
would further increase the efficacy of rTMS. When using these ultra-low intensities, following the Arnold tongue demands to adjust stimulation frequency and location increase substantially. Otherwise, the intervention will likely to miss effects.

It is unclear whether the observed changes in neural synchronization would manifest in observable behavioral effects. A candidate mechanism through which such changes could manifest is increased cortical inhibition via increased neural synchrony. In general, at low intensities inhibitory circuits seem to be stimulated preferentially ${ }^{115,116}$. Also, alpha activity has been associated with cortical inhibition ${ }^{117-119}$, thus, hyperpolarization instead of excitation may play a decisive role.

\subsection{Methods}

\subsubsection{Participants}

We recruited neurologically healthy volunteers in this study (see Table 1). We included participants, if we could estimate the individual alpha frequency in the eyes closed or open resting state conditions. In the main experiment, the dataset of one was incomplete and was excluded from further analysis. We used the Edinburgh Handedness Inventory ${ }^{120}$ to estimate the laterality index of our participants. The sample size was determined based on earlier rTMS-EEG studies ${ }^{40,86,93}$.

\begin{tabular}{lll}
\hline & Main experiment & Control experiment \\
Final sample size & 16 & 16 \\
Excluded participant(s) & 1 & 0 \\
Mean age \pm SD (years) & $25.5 \pm 3.2$ & $23.9 \pm 3.9$ \\
Age range (years) & from 21 to 32 & from 20 to 34 \\
Number of women/men & $8 / 8$ & $8 / 8$
\end{tabular}



Mean laterality index \pm SD
$78.4 \pm 50.1$
$78.8 \pm 31.6$
Laterality index range
from -30 to 100
from 0 to 100

Table 1. The participant information in the main and control experiments. In the main experiment, the dataset of one participant was incomplete and was excluded from further analysis.

Before participation, all volunteers filled out self-completed questionnaires to assess the study exclusion criteria. In cases of possible contraindications, a neurologist at the Department Clinical Neurophysiology, University Medical Center Göttingen examined the volunteer. Inclusion criteria were no history or presence of medical, neurological or psychiatric illnesses including epilepsy, drug and/or alcohol abuse, and no metal implants in the head, neck, or chest.

\subsubsection{Ethic statement and research integrity}

The Ethic Committee of the University Medical Center Göttingen approved the investigation, the experimental protocols, and all methods (Application number: 35/7/17). We performed all experiments in accordance with relevant guidelines and regulations. All participants gave written informed consent before participation. The raw data and code for the reported analyses are available for download at our repository (https://github.com/ZsoltTuri/2019 rTMS-EEG).

\subsubsection{Procedure}

In the main experiment, the participants took part in one neuroimaging session were we collected anatomical, diffusion weighted and functional magnetic resonance imaging data. In the next session, we estimated the resting motor threshold by using neuronavigated single pulse TMS. Preceding the three rTMS-EEG sessions, we performed the head modeling and EF calculations to 
prospectively estimate the stimulation intensity for each individual and session. In the control experiment, the participant took part in one rTMS-EEG session. Unknown to the participants, they received only sham rTMS.

\subsubsection{Acquisition and analysis of neuroimaging data}

Acquisition. Magnetic resonance images (MRI) were acquired using a 3T MRI-scanner (Siemens Magnetom TIM Trio, Siemens Healthcare, Erlangen, Germany) equipped with a 32-

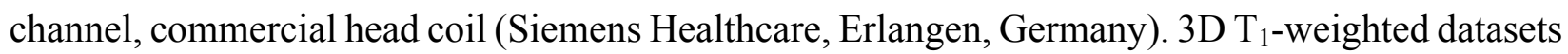
were obtained using Magnetisation Prepared RApid Gradient Echo (MP-RAGE) acquisitions with or without selective water excitation for fat suppression employing the following parameters: Turbo fast low angle shot (Turbo FLASH), echo time (TE): $3.26 \mathrm{~ms}$, repetition time (TR): 2250 ms, inversion time: $900 \mathrm{~ms}$, flip angle: $9^{\circ}$, receiver bandwidth: $200 \mathrm{~Hz} / \mathrm{Px}$ that cover the whole head at $1 \times 1 \times 1 \mathrm{~mm}^{3}$ isotropic resolution.

3D $\mathrm{T}_{2}$-weighted Turbo spin echo (TurboSE) sequences were acquired with and without fat suppression using the following imaging parameters: TE: $282 \mathrm{~ms}$, TR: $3500 \mathrm{~ms}$, slice number: 176, slice thickness: 1mm, field of view (FoV; longitudinal coverage): $256 \mathrm{~mm}$, echo spacing: $4.84 \mathrm{~ms}$, turbo factor 125 , receiver bandwidth: $355 \mathrm{~Hz} / \mathrm{Px}$ that cover the whole head at $1 \times 1 \times 1 \mathrm{~mm}^{3}$ isotropic resolution.

For diffusion-weighted imaging, single-shot spin-echo echo planar imaging sequences were obtained using the following parameters: TE: $88 \mathrm{~ms}$, TR: $10.000 \mathrm{~ms}$, slice thickness: $1.7 \mathrm{~mm}$, FoV longitudinal coverage: $218 \mathrm{~mm}$, receiver bandwidth: $1346 \mathrm{~Hz} / \mathrm{Px}$. For accelerating $\mathrm{T}_{2^{-}}$and diffusion-weighted image acquisitions, we obtained parallel imaging techniques by means of 
generalized autocalibrating partially parallel acquisitions (GRAPPA) with a twofold acceleration factor.

In addition, the participant performed rhythmic, stereotypic movements with the first dorsal interosseous muscle, i.e. thumb adduction, to localize its cortical representation. We used a gradient-echo planar imaging (EPI) sequence to detect BOLD changes by using the following imaging parameters: TR/TE: $900 \mathrm{~ms} / 30 \mathrm{~ms}$, flip angle $=50^{\circ}$, voxel size $3 \times 3 \times 3 \mathrm{~mm}^{3}$, field of view (FOV) $210 \times 210 \mathrm{~mm}, 39$ slices (whole brain) and 284 volumes.

Analysis of the fMRI data. Raw DICOM images were converted to NIfTI format using the software MRIConvert (2.1.0). The fMRI data preprocessing was performed with the Statistical Parametric Mapping (SPM 12, Welcome Department of Imaging Neuroscience, London, UK) software package implemented in the Matlab environment. Following slice-timing correction, functional images were realigned to the first volume by affine registration using the standard, six parameters rigid body spatial transformation method. EPI volumes were then co-registered to the $1 \mathrm{~mm}$ isotropic $\mathrm{T}_{1}$ anatomical image, which had been previously reoriented to the anterior commissure. EPI volumes were spatially smoothed using Gaussian kernel of $6 \mathrm{~mm}$ full width at half maximum.

Following preprocessing, statistical analysis was performed at the single-subject level in the framework of the general linear model. Voxels were identified as significant if $p<0.05$ (familywise error corrected for multiple comparisons on the voxel level). We used this parametric map to position our TMS coil in the motor threshold hunting session. 


\subsubsection{Neuronavigated (r)TMS}

We used neuronavigated (r)TMS in the main experiment in the motor threshold hunting session and 2) in the subsequent rTMS-EEG sessions. In both, i.e., main and control, experiments, we used a MagPro X100 stimulator (MagVenture, Denmark) with a standard figure-eight coil (MC-B70) to deliver biphasic single and repetitive TMS pulses with the normal coil current direction (280 $\mu \mathrm{s}$ pulse width). During stimulation, the participants sat in a fixed chair equipped with an armrest and their eyes open. We used an in-house built, light aluminum frame structure, equipped with softcushioned bilateral head fixation pads and chin rest. We mounted the TMS coil on a variable friction arm (Model 244N, Manfrotto, Italy) and adjusted the position of each element individually to achieve the required comfort of the participant and precise TMS targeting in the spTMS and rTMS sessions.

To accurately guide the TMS coil over the anatomical target in the main experiment, we used a frameless, stereotactic MRI-based real-time neuronavigation system (Brainsight TMS Navigation, Rogue Resolutions Ltd) and coupled it with a Polaris Vicra infrared camera (NDI, Waterloo, Canada). In the motor threshold determination session, the target of spTMS was the motor cortex representation of the first dorsal interosseous muscle, which we had previously identified as the highest of the fMRI local activation maximum derived from the parametric t-map at the anatomical hand knob formation.

In the rTMS-EEG sessions (both experiments), the target location was at the PO3 electrode. In the main experiment, we employed neuronavigation for real-time monitoring of the coil location over the target (within $2 \mathrm{~mm}$ ) and for recording the coil location, which permitted consistent targeting in subsequent rTMS-EEG sessions. To achieve optimal targeting, we did not completely 
fix the position of the adjustable coil support, which enabled the investigator to make a small adjustment to the coil position in real-time.

\subsubsection{Determination of motor threshold}

In the main experiment, we recorded the surface electromyogram from the right first dorsal interosseous muscle with an $\mathrm{Ag}-\mathrm{AgCl}$ electrode pair in a belly-tendon montage. Raw signals (sampling rate $5 \mathrm{kHz}$ ) were amplified, band-pass filtered between $2 \mathrm{~Hz}$ and $3 \mathrm{kHz}$ and digitized with a micro 1401 AD converter (Cambridge Electronic Design, Cambridge, UK). Data collection was controlled by Signal Software (Cambridge Electronic Design, version 4.08).

The EEG electrode array was already in position during the motor threshold hunting session, in order to keep a constant scalp-coil distance in the motor threshold hunting and the subsequent rTMS-EEG sessions. This was a necessary step because the TMS-induced magnetic field (and hence the induced EF) changes as the inverse cube of the distance. No simultaneous EEG recordings were performed during the motor threshold hunting session.

We searched for the anatomical hot spot for the target muscle by initially positioning the coil over the scalp projection area of the fMRI peak voxel with the highest statistical t-value using the neuronavigation system. We then determined the optimal coil position (orientation, angle) in which spTMS elicited the strongest motor evoked potentials (MEP) in the target muscle.

Following optimal positioning, we estimated the RMT by determining the minimum stimulation intensity, expressed as a percentage of MSO, with which at least three out of six TMS pulses produced MEPs with $\mathrm{a} \geq 50 \mu \mathrm{V}$ peak-to-peak amplitude in the resting target muscle ${ }^{121}$. Motor threshold hunting started with an intensity of 30\% of MSO that was gradually increased in $5 \%$ steps until spTMS consistently evoked MEPs with $\geq 50 \mu \mathrm{V}$ amplitude. Thereafter, we reduced 
the stimulation intensity in 1\% steps until the RMT was estimated. During the entire procedure, we encouraged the participants to indicate the presence of perceivable effects, such as discomfort due to cranial muscle activation or dizziness, and to inform the investigator if the stimulation was not tolerable. Because of tolerability issues, we set a limit for the stimulation intensity at $75 \%$ of MSO. If RMT was not detected by then, it was labeled "undefined" $(n=3)$. The RMT $(n=14)$ was on average $54.79 \% \pm 12.29 \%$ (SD) MSO.

\subsubsection{Head modeling and EF calculations}

We performed individual high-resolution, anatomically realistic head modeling and EF calculations using the Simulation of Non-invasive Brain Stimulation (SimNIBS) software package 122. These calculations were performed twice: The first time to determine the dose for rTMS (at the single-subject level), and the second time to retrospectively estimate the spatial characteristics and magnitude of the rTMS-induced EF at the group-level. We used the SimNIBS versions 2.0.1 and 2.1.2 for the prospective and retrospective EF calculations, respectively. For both calculations, we set the scalp-to-coil distance to $11 \mathrm{~mm}$ in order to take the EEG electrodes into consideration.

The mri2mesh function automatically generated tetrahedral volume meshes of the head from $\mathrm{T}_{1}$ - and $\mathrm{T}_{2}$-weighted structural MR images ${ }^{123}$. The final head mesh consisted of approx. 3.500.000 tetrahedral elements and five tissue compartments. Table 2 shows the five tissue compartments and their conductivity values. We performed EF calculations using the finite element method and the built-in MC-B70 validated coil.

\begin{tabular}{ll}
\hline Tissue & Conductivity \\
compartment & {$[\mathrm{S} / \mathrm{m}]$} \\
Scalp & 0.465 \\
Skull & 0.010 \\
\hline
\end{tabular}




\begin{tabular}{ll}
\hline CSF & 1.654 \\
$\mathrm{GM}^{*}$ & 0.275 \\
$\mathrm{WM}^{*}$ & 0.126 \\
\hline
\end{tabular}

Table 2. The five tissue compartments of the head model and their conductivity values $[\mathrm{S} / \mathrm{m}]$. Abbreviations: CSF - cerebrospinal fluid, GM-gray matter, WM - white matter. Asterisks indicate anisotropic conductivity values estimated from diffusion tensors using the volume-normalized approach in the retrospective EF calculations ${ }^{124}$.

The goal of the second, retrospective computation was to compare the resulting EFs of the currently used, near threshold approach to our novel approach. For this, we used the maximum stimulator output data derived from the motor threshold determination and rTMS-EEG sessions. In the retrospective computation, we used improved procedures for creating individual head models by assigning anisotropic values to the gray and white matter compartments. We furthermore performed a region of interest (ROI) analysis by focusing on the EF in the parietal and occipital regions.

We also characterized the magnitude of the absolute EF as well as of its normal component, since there is a current debate about the physiological effects of the spatial components of the EF 105,106. The normal component of the EF can distinguish the depolarizing inward and hyperpolarizing outward EFs ${ }^{125}$. There are claims that the normal component in the wall of the motor cortex is the physiologically effective constituent in the induction of motor evoked potentials in humans ${ }^{46}$. However, considerable skepticism remains about the physiological efficacy of the normal component of the $\mathrm{EF}^{46,105,106}$. For example, the peak values of the absolute $\mathrm{EF}$ in the gyral crown of the motor cortex is a similarly plausible component in producing a liminal response in 
the motor cortex ${ }^{105}$. Given this uncertainty in the TMS literature we analyzed both the absolute EF and its normal component.

In the group-level analysis, we provide the peak $\left(99.9^{\text {th }}\right.$ percentile), median and mean EF values. In the group-level ROI analysis, first, we calculate the mean EF strength for each ROI separately at the single-subject level. Then, we calculate median, mean and 95\% CIs at the grouplevel.

\subsubsection{EEG acquisition}

We performed EEG recordings 1) to estimate the individual alpha band peak frequency (IAF) and 2) to characterize the immediate electrophysiological effects and short-lasting aftereffects of rTMS. We attained scalp EEG data with a 24-bit, battery-powered, active channel amplifier with $64 \mathrm{Ag} / \mathrm{AgCl}$ active EEG electrodes (actiCAP, BrainVision LLC, Germany) at a $2.5 \mathrm{kHz}$ sampling rate, and without hardware filters (actiChamp, Brain Vision LLC, Germany). Ground and reference electrodes were located at Fpz and FCz, respectively. Impedance values were maintained below 20 $\mathrm{k} \Omega$.

\subsubsection{Estimating individual alpha band peak frequencies}

In both experiments, we estimated IAF at the beginning of each rTMS-EEG session in order to fine-tune the stimulation frequency in the rhythmic rTMS condition. We recorded two four-minute blocks of continuous, resting state EEG, one block with eyes open and the other with eyes closed. We instructed our participants to sit calmly, stay relaxed, not to move their limbs or face muscles, and try to avoid any repetitive mental activity such as reproducing any texts, lyrics or melodies. 
Participants wore QuietControl 30 wireless headphones with active noise reduction and proper earbud size during the EEG recordings (Bose Corporation, USA).

We performed offline data analysis with the FieldTrip toolbox for EEG- and MEG analysis [version 20170119; 85; http://fieldtrip.fcdonders.nl]. The data was initially segmented into $2 \mathrm{~s}$ epochs with $50 \%$ overlap, re-referenced to the common average, detrended, demeaned, high-pass $(0.1 \mathrm{~Hz})$ and low-pass $(40 \mathrm{~Hz})$ filtered with an infinite impulse response filter type Butterworth. The trials were visually inspected for outliers and were rejected based on variability.

Frequency analysis between 1 and $20 \mathrm{~Hz}$ with $0.5 \mathrm{~Hz}$ increments was performed with the multitaper frequency transformation ('mtmfft') method based on discrete prolate spheroidal sequences. After averaging over trials, we determined peak alpha power (and IAF) in the range from 8 to $12 \mathrm{~Hz}$ by visual inspection for both "eyes open" and "eyes closed" condition. However, if we could not determine peak alpha frequency from the eyes open condition, we used the eyes closed condition instead (five cases). The average IAF was $10.3 \pm 1.0 \mathrm{~Hz}$.

\subsubsection{Simultaneous rTMS and EEG}

In each rTMS-EEG session of the main experiment, we employed rhythmic (main) and arrhythmic (active control) rTMS protocols over the same target location with the same stimulation

intensity ${ }^{40,86}$. In the control experiment, we employed a single rhythmic sham rTMS protocol by tilting the coil with $90^{\circ}$ angle.

In the rhythmic protocol, we set the pulse repetition frequency at IAF. The stimulation frequency and the number of pulses for the rhythmic rTMS burst were preprogrammed in the TMS device, and the start of each rTMS burst was controlled externally via PsychoPy (version 1.83.01) using a parallel port and a Bayonet Neill-Concelman connector ${ }^{127,128}$. 
In the arrhythmic protocol, we set a pseudorandom stimulation frequency, and the delivery of each pulse was controlled externally via PsychoPy. Despite pseudorandomization, rhythmic patterns in the alpha frequency band can still emerge by chance for at least several consecutive rTMS pulses ${ }^{86}$. Therefore, we predefined the timing of the TMS pulses so that frequencies in the alpha frequency band $(8-12 \mathrm{~Hz})$ and their harmonics and subharmonics were not allowed to occur between the pulses (for instance 4 and $16 \mathrm{~Hz}$ for $8 \mathrm{~Hz} ; 4.5 \mathrm{~Hz}$ and $18 \mathrm{~Hz}$ for $9 \mathrm{~Hz} ; 5$ and $20 \mathrm{~Hz}$ for $10 \mathrm{~Hz} ; 5.5 \mathrm{~Hz}$ and $22 \mathrm{~Hz}$ for $11 \mathrm{~Hz} ; 6$ and $24 \mathrm{~Hz}$ for $12 \mathrm{~Hz}$ ).

Each rTMS burst contained 20 pulses and each block contained 25 rTMS bursts. The interburst interval was randomly selected to be 10s or 11s. In each rTMS-EEG session, we delivered five rhythmic and five arrhythmic blocks, the order of which was randomized for each session and participant.

In the main experiment, the intensity of the rTMS burst was set based on the desired, prospectively estimated EF strength. In the control experiment, we used a fixed stimulation intensity at $29 \%$ of the device output. Apart from the stimulation intensity and the coil angle, all the remaining stimulation parameters were the same in the real rhythmic protocols (main experiment) and the sham rhythmic protocol (control experiment).

All stimulation protocols were performed with the participants at rest and instructed to keep their eyes open. During each block, a white noise, auditory masking stimulus was delivered through the QuietControl 30 wireless earphones. At the beginning of each session, we asked the participants to indicate the maximum sound volume that they could tolerate for more than an hour. The sound volume of the white noise was always kept below the manufacturer's recommended safety limits. This procedure minimized but did not completely eliminate the participants' ability to hear the click sound produced by the discharge of the coil ${ }^{70,129}$. 


\subsubsection{Analysis of rTMS-EEG}

EEG preprocessing. We performed offline data analysis using the FieldTrip toolbox (v.20180114, http://fieldtrip.fcdonders.nl) with a custom-made MATLAB code. We first segmented the EEG data into trials of 8.5 seconds length that were time-locked to the offset of the rTMS burst in an interval from $3.5 \mathrm{~s}$ before to $5 \mathrm{~s}$ after the last TMS pulse. In the main experiment, each session contained ten blocks of both rhythmic and arrhythmic stimulation. We appended all blocks from a session into one data file and performed the preprocessing blinded to the stimulation type. Each dataset contained 125 trials for the rhythmic and 125 for the arrhythmic stimulation condition. The sham stimulation session contained 125 trials. All the following steps were identical in the main and control experiment.

We defined a ringing artifact of the TMS pulse as the time interval from $4 \mathrm{~ms}$ before to $9 \mathrm{~ms}$ after the pulse and excised them from the data. As the data still contained residual decay artifacts we ran an independent component analysis (fastICA) with 63 components. To define which components corresponded to decay artifacts we averaged the components' signal $50 \mathrm{~ms}$ after TMS pulse over all trials. We rejected components whose amplitude exceeded $30 \mu \mathrm{V}$. On average, $0.5 \pm 1.2$ (Low); $1.2 \pm 1.2$ (Medium); $1.3 \pm 1.2$ (High) components were defined as decay artifacts. The time intervals around the TMS pulse were replaced via Piecewise Cubic Hermite Interpolation (pchip).

The data then was re-referenced to the common average and was down-sampled to 1,250 Hz. We inspected the data to determine which channels or trials contained artifacts using a semiautomatic algorithm adapted from ARTIST ${ }^{95}$ that contained three main criteria. First, we estimated the power of each trial. The outlier power values for each trial and channel were defined as elements lying more than 1.5 interquartile ranges above the upper quartile or below the lower quartile. We 
marked trials or channels that corresponded to outlier values as 'bad'. If the number of 'bad' channels was less than $20 \%$ of the total amount, we interpolated the channels only within the 'bad' trial using the weighted signal of neighbor channels. If the percentage was higher, the trial was rejected. We then removed channels with a large standard deviation $(\mathrm{STD}>30 \mu \mathrm{V})$, which was related to channel movements under the TMS coil. In the next step, we defined channels with line noise or high impedance values. For that, we estimated the correlation coefficient of the signal from a channel with the signal of its neighbors. We removed channels with a low correlation coefficient (CorrCoef $<0.4$ ). On average, 4.2 \pm 1.8 (Low), 3.8 \pm 1.9 (Medium), 3.8 \pm 2.1 (High) channels were interpolated using the weighted signal from neighbor channels.

As the last preprocessing step, we defined eye-related artifacts via the second ICA run. We reduced the number of independent components due to interpolated channels. Components corresponding to blinks, saccades and other eye movements were rejected. On average, we rejected 3.1 \pm 1.8 (Low), $2.9 \pm 1.3$ (Medium), and 2.8 \pm 1.2 (High) components during the second ICA run. For

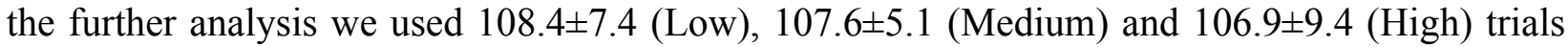

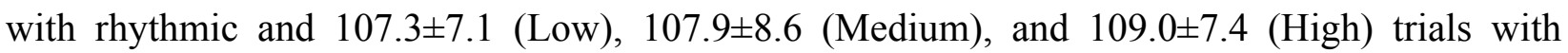
arrhythmic stimulation.

Phase locking value. We chose the PLV to describe the degree of synchronization of the EEG signal by an external repetitive force. PLV is based on the measurement of phase alignment of the signal to external pulses or stimulated channels ${ }^{130}$.

First, we simulated the sinusoidal wave as an additional channel for each trial at the individual alpha frequency. The phase of the simulated wave was aligned to the TMS offset. The data was decomposed by complex Morlet wavelet. The wavelets contained five cycles with a three- 
Gaussian window. The decomposition was performed for the trial interval from 3.5 before to $2.5 \mathrm{~s}$ after TMS offset.

Then, we computed PLV between the phase component of the simulated wave with the remaining channels as follow:

$$
P L V=\frac{1}{N}\left|\sum_{n=1}^{N} e^{i\left(\Phi_{\text {ong }}(n)-\Phi_{I A F}(n)\right)}\right|
$$

where $\mathrm{N}$ is the number of repetitions (trials), $\Phi_{\text {ong }}(\mathrm{n})$ and $\Phi_{\mathrm{IAF}}(\mathrm{n})$ are the instantaneous phase values at the time points $\mathrm{n}$ of the ongoing EEG signal and simulated sinusoidal wave at IAF, respectively. The values range from 0 to 1 . The PLVs were baseline-normalized by dividing the values at each sample by the value average during the 3.0 to 2.5 seconds prior to TMS offset (In Fieldtrip: relative normalization).

Statistical analysis. Differences in PLVs between rhythmic, arrhythmic and sham stimulation conditions were subjected to cluster-based permutation statistical analysis (two-tailed) at the respective stimulation frequency $(8-12 \mathrm{~Hz})$. In the main experiment, we used the dependent t-test between to compare the rhythmic and arrhythmic conditions at the Low, Medium and High sessions separately. In addition, we used the independent t-test to compare the real rhythmic conditions with the control (sham) condition. To control for multiple comparisons, we applied a non-parametric randomization approach. This procedure uses 1,000 randomizations to estimate the probability that a given number of significant electrodes $(\mathrm{p}<0.05)$ can be expected by chance.

Control analyses. In order to ensure that the steps used in the rTMS-EEG data analysis cannot account for the observed pattern of findings we performed an additional control experiment by stimulating a piece of meat (chicken, phantom). Moreover, we used our control dataset consisting of artifact-free resting state EEG data to study whether our preprocessing pipeline can spuriously 
induce the degree of neural synchronization. For both analyses, we used the identical pipeline for data preprocessing and PLV analysis, as described for the main analyis.

In the phantom experiment, rhythmic rTMS was applied at the highest intensity used in the main group and sham group $-29 \%$ of maximum stimulator output. Pulses were applied in five blocks at stimulation frequency $10 \mathrm{~Hz}$. The data were recorded from 32 EEG channels.

The control dataset was taken from EEG data recorded from 16 participants before each rTMS-EEG session with the initial aim of estimating the individual alpha frequencies (IAF). The data contain a realistic amount of noise with realistic time-frequency characteristics. Because we applied no rTMS during resting state EEG data recordings, we simulated TMS pulses by periodically removing data segments and interpolating them using the IAF.

Data segments of lengths comparable to the main dataset were marked with arbitrary 'TMS pulse' onsets based on IAF. The three sessions from each participant were appended to one dataset in order to increase the number of trials. An average of $104.2 \pm 6.5$ trials were used for the analysis. This is comparable with the trial numbers used in the main analysis (for example, $108.4 \pm 7.4$ for low intensity).

\subsection{Supplementary material}

\subsubsection{Validation measurements}

Since rTMS devices typically operate at high stimulation intensities ( $\geq 30 \% \mathrm{MSO})$ we first validated the output stability of our MagPro X100 stimulator with the MC-B70 coil in the lower stimulation intensity regimen. For that purpose, we measured the induced EF waveforms and peak voltage values with an external induction coil, which was connected to a digital storage 
oscilloscope (Rigol DS1052E). The tests were performed at intensities from five to 20 percent of MSO with $1 \%$ increments and at $30 \%$ of MSO. We applied 20 TMS pulses at each pre-determined stimulation intensity. The stimulator produced detectable and stable TMS pulses starting at $8 \%$ of MSO. This prospective validation measurement determined the practical lower limit for the rTMS dose, and we ensured that all participants received a stimulation intensity of $\geq 8 \% \mathrm{MSO}$. This value corresponds to the induced peak value of an absolute EF strength of ca. $20 \mathrm{mV} / \mathrm{mm}$. Note that TMS pulses weaker than $8 \%$ MSO probably produced electromagnetic-fields but our external induction coil was unable to detect them.

\begin{tabular}{|r|l|l|c|c|c|}
\hline Index & PMID & \multicolumn{1}{|c|}{ Article } & Method & $\begin{array}{c}\text { Threshold } \\
\text { percent }\end{array}$ & MSO \\
\hline 1 & 28343866 & Albouy et al. $^{86}$ & FXD & i/r & 60 \\
\hline 2 & 29247630 & Ando et al. $^{131}$ & RMT & 90 & $\mathrm{n} / \mathrm{r}$ \\
\hline 3 & 27812319 & Bai et al. $^{132}$ & RMT & 90 & $\mathrm{n} / \mathrm{r}$ \\
\hline 4 & 28928648 & Bharath et al. $^{133}$ & RMT & 90 & $\mathrm{n} / \mathrm{r}$ \\
\hline 5 & 29241839 & Cao et al. $^{134}$ & RMT & 100 & 44.5 \\
\hline 6 & 27600845 & Capotosto et al. $^{135}$ & RMT & 100 & $\mathrm{n} / \mathrm{r}$ \\
\hline 7 & 30099627 & Cha et al. $^{136}$ & RMT & 110 & $\mathrm{n} / \mathrm{r}$ \\
\hline 8 & 29060275 & Chen et al. $^{137}$ & RMT & 110 & $\mathrm{n} / \mathrm{r}$ \\
\hline 9 & 27445730 & D'Agata et al. $^{138}$ & RMT & 80 & $\mathrm{n} / \mathrm{r}$ \\
\hline 10 & 27215619 & Daltrozzo et al. $^{139}$ & RMT & 90 & $\mathrm{n} / \mathrm{r}$ \\
\hline 11 & 26679060 & DelFelice et al. $^{140}$ & RMT & 100 & $58.6 / 61.9$ \\
\hline 12 & 30253222 & DiGiacomo et al. $^{141}$ & RMT & 80 & 47.4 \\
\hline 13 & 27626224 & Emrich et al. $^{142}$ & RMT & 110 & 72 \\
\hline 14 & 29984172 & Fisher et al. $^{143}$ & RMT & 90 & $\mathrm{n} / \mathrm{r}$ \\
\hline 15 & 26608023 & Gongora et al. $^{144}$ & RMT & 80 & 47.4 \\
\hline 16 & 29770146 & He et al. $^{145}$ & RMT & 100 & $\mathrm{n} / \mathrm{r}$ \\
\hline 17 & 29224411 & Hunter et al. $^{146}$ & RMT & $80-120$ & $\mathrm{n} / \mathrm{r}$ \\
\hline 18 & 29238296 & Jin et al. $^{147}$ & RMT & 90 & $\mathrm{n} / \mathrm{r}$ \\
\hline 19 & 26778629 & Kamp et al. $^{148}$ & RMT & 110 & $\mathrm{n} / \mathrm{r}$ \\
\hline 20 & 28413707 & Karton et al. $^{149}$ & vMT & 80 & $\mathrm{n} / \mathrm{r}$ \\
\hline 21 & 27138833 & Kazemi et al. $^{150}$ & RMT & $100 / 120$ & $\mathrm{n} / \mathrm{r}$ \\
\hline 22 & 30233346 & Kazemi et al. $^{151}$ & RMT & $100 / 120$ & $\mathrm{n} / \mathrm{r}$ \\
\hline 23 & 30386222 & Keuper et al. $^{152}$ & FXD & i/r & 50 \\
\hline 24 & 27909453 & Kim et al. $^{153}$ & vMT & 110 & $\mathrm{n} / \mathrm{r}$ \\
\hline & & & & \\
\hline
\end{tabular}




\begin{tabular}{|r|l|l|c|c|c|}
\hline 25 & 27852164 & Kito et al. $^{154}$ & MT & 120 & $\mathrm{n} / \mathrm{r}$ \\
\hline 26 & 29277405 & Koch et al. $^{155}$ & RMT & 110 & 60.8 \\
\hline 27 & 25165064 & Li et al. $^{156}$ & MT & 100 & $\mathrm{n} / \mathrm{r}$ \\
\hline 28 & 28959194 & Li et al. $^{157}$ & RMT & 110 & $\mathrm{n} / \mathrm{r}$ \\
\hline 29 & 28614399 & Li et al. $^{158}$ & RMT & 110 & $\mathrm{n} / \mathrm{r}$ \\
\hline 30 & 29742385 & Lowe et al. $^{159}$ & RMT & 80 & $52 / 53$ \\
\hline 31 & 28689295 & Lozeron et al. $^{160}$ & RMT & 80 & $\mathrm{n} / \mathrm{r}$ \\
\hline 32 & 28008080 & Moebius et al. $^{161}$ & RMT & 110 & $\mathrm{n} / \mathrm{r}$ \\
\hline 33 & 30219485 & Nathou et al. $^{162}$ & RMT & 80 & $\mathrm{n} / \mathrm{r}$ \\
\hline 34 & 27516735 & Nicolo et al. $^{163}$ & vMT & 90 & $\mathrm{n} / \mathrm{r}$ \\
\hline 35 & 28160748 & Noda et al. $^{154}$ & RMT & 95 & 82.2 \\
\hline 36 & 30318052 & Noda et al. $^{164}$ & RMT & 95 & 78 \\
\hline 37 & 26873935 & Oshima $^{165}$ & RMT & 90 & $\mathrm{n} / \mathrm{r}$ \\
\hline 38 & 30290037 & Prashad et al. $^{166}$ & vMT & 80 & $\mathrm{n} / \mathrm{r}$ \\
\hline 39 & 29914282 & Rocha et al. $^{167}$ & RMT & 80 & 46.2 \\
\hline 40 & 26584867 & Romei $^{93}$ & AMT & 90 & 41.9 \\
\hline 41 & 27687560 & Rousseau $^{168}$ & RMT & 120 & $\mathrm{n} / \mathrm{r}$ \\
\hline 42 & 30425640 & Shalbaf et al. $^{169}$ & vMT & 120 & $\mathrm{n} / \mathrm{r}$ \\
\hline 43 & 29249371 & Shields et al. $^{170}$ & vMT & 90 & $\mathrm{n} / \mathrm{r}$ \\
\hline 44 & 28539601 & Spadone et al. $^{171}$ & RMT & 100 & $\mathrm{n} / \mathrm{r}$ \\
\hline 45 & 27428476 & Tikka et al. $^{172}$ & RMT & 80 & $\mathrm{n} / \mathrm{r}$ \\
\hline 46 & 30295684 & Valiulis et al. $^{173}$ & MT & 100 & $\mathrm{n} / \mathrm{r}$ \\
\hline 47 & 28902713 & Xia et al. $^{174}$ & RMT & 90 & $\mathrm{n} / \mathrm{r}$ \\
\hline
\end{tabular}

Table 3. The vast majority of rTMS studies determine stimulation intensity with the so-called near threshold dosing approach

We performed a systematic search on PubMed of literature published between 2016 and 2018 with the searching terms "rTMS AND EEG" and "rhythmic TMS AND EEG". Of the 134 hits, we found 47 eligible articles. All published studies had determined the stimulation frequency with the fixed intensity or the motor threshold approach. The articles are ordered alphabetically. Abbreviations: FXD: fixed intensity; i/r: irrelevant; MSO: maximum stimulator output expressed in percentages; MT: motor threshold; n/r: information is not reported; PMID: PubMed identification number; RMT: resting motor threshold; vMT: visually identified motor threshold. 


\section{Absolute electric field}

A

Spatial distribution

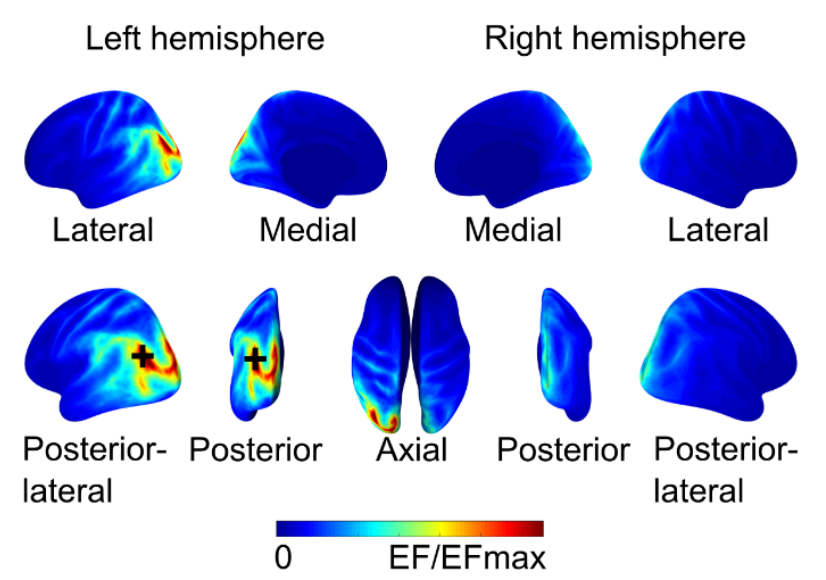

B

Peak magnitudes

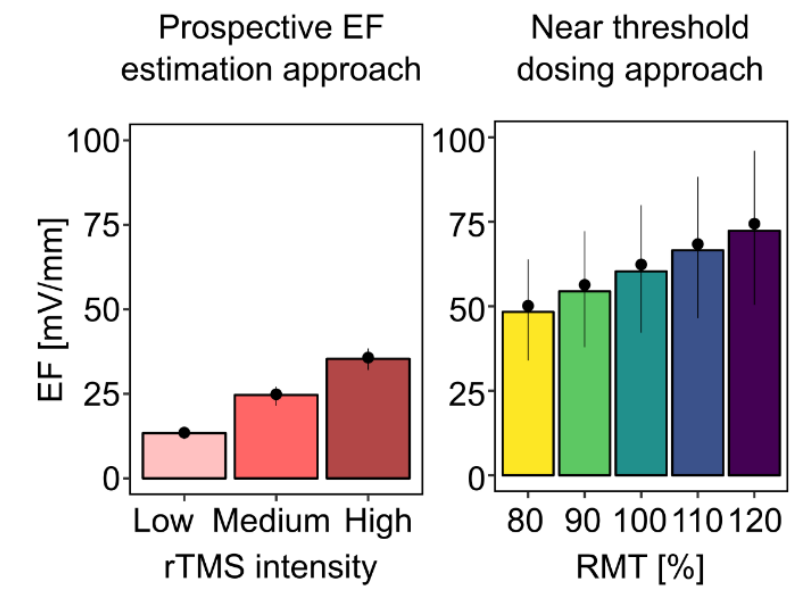

\section{Normal component of the electric field}

C

Spatial distribution

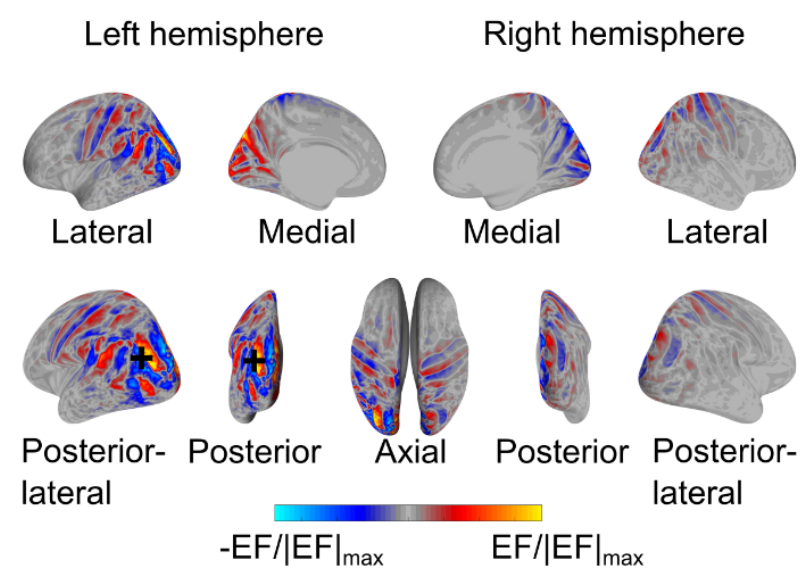

D

Peak magnitudes

Prospective EF estimation approach

Near threshold dosing approach
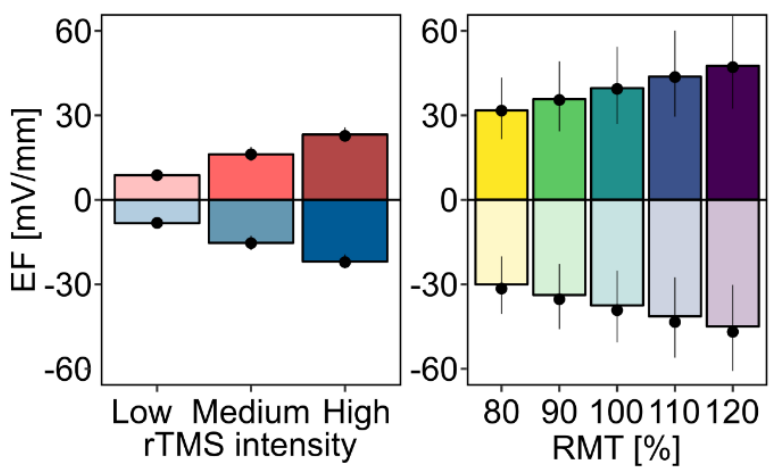

Figure 10. Group-level $(n=16)$ spatial distribution of electric field values. (A) Absolute electric field values were extracted from the gray matter and projected onto the inflated Freesurfer average template brain. (B) Group-level peak magnitudes of the absolute electric field values in the two rTMS dosing approaches. (C) Group-level normal component of the electric field values was extracted from the gray matter and projected onto the inflated Freesurfer average template brain. (D) Peak magnitudes of the normal component of the electric field in the two rTMS dosing approaches. Peak magnitudes correspond to the 99.9th percentile. A black plus sign shows the 
positioning of the TMS coil over the PO3 electrode. Bar plots show the mean and dot plots show the median values. Range plots correspond to the 2.5th and the 97.5th percentiles, respectively. Abbreviations: EF-electric field; RMT-resting motor threshold.
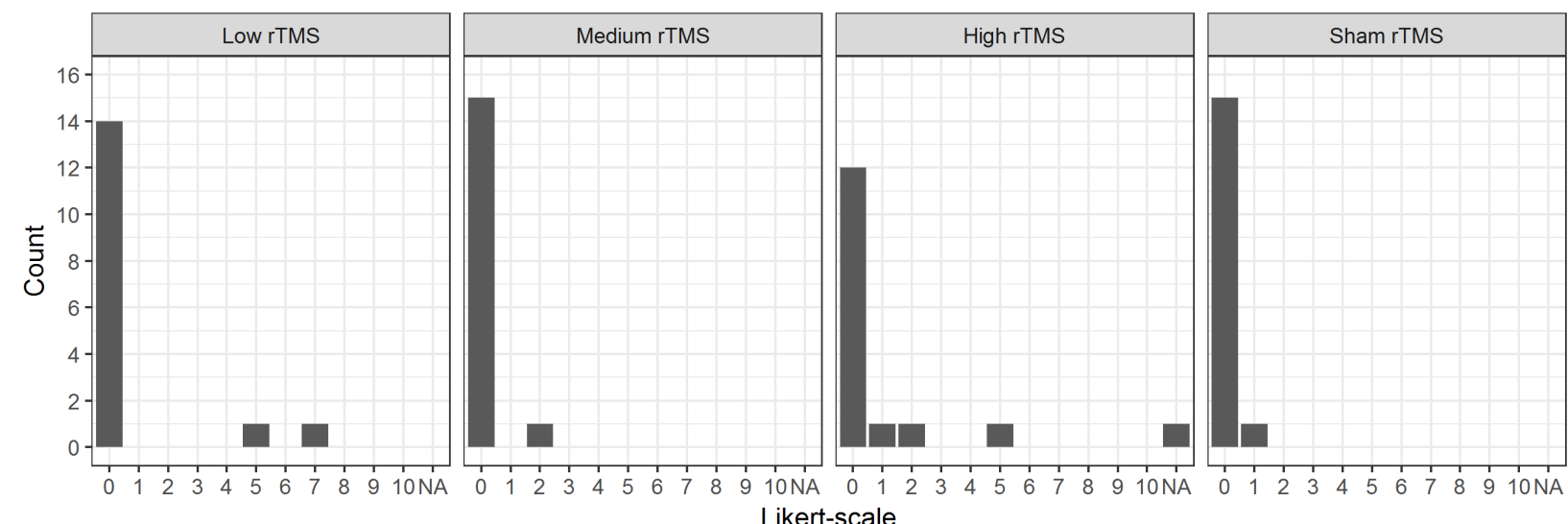

Figure 11. Participants reported a minimal amount of somatosensory perceptual adverse effects. Both in the main (low, medium and high rTMS) and control experiment (sham rTMS), the participants filled out a post-experimental questionnaire about the somatosensory perceptual adverse effects. Likert scale ranges from 0 and 10, where 0 refers to no sensation detected, 1 indicates minimally detectable sensation and 10 refers to unbearably uncomfortable sensation. NA refers to the case when no answer was provided by the participant. 


\title{
4. Short-lived alpha power suppression induced by low-intensity arrhythmic rTMS
}

\author{
Elina Zmeykina ${ }^{1}$, Matthias Mittner ${ }^{2}$, Walter Paulus ${ }^{1}$ and Zsolt Turi* ${ }^{1,3}$ \\ ${ }^{1}$ Department of Clinical Neurophysiology, University Medical Center Goettingen, Göttingen, \\ Germany \\ ${ }^{2}$ Department of Psychology, University of Tromsø, Norway, \\ ${ }^{3}$ Institute of Anatomy and Cell Biology, University of Freiburg, German
}

Neuroscience, under review

Preprint: Biorxiv DOI: $\underline{\text { https://doi.org/10.1101/2020.10.28.358986 }}$

\section{Author contributions:}

E.Z.: study design, formal analysis, project administration, investigation, methodology, software, visualization, data interpretation, data curation, and writing original draft.

M.M.: study design, supervision and collaboration writing original draft.

W.P.: study design, funding acquisition, resources, project administration, supervision, and collaboration writing original draft.

Z.T.: conceptualization, study design, funding acquisition, project administration, investigation, methodology, software, visualization, and writing original draft.

Copyright (C) 2020, The Author(s). This is an open access article distributed under the terms of the Creative Commons CC BY license, which permits unrestricted use, distribution, and reproduction in any medium, provided the original work is properly cited. 


\subsection{Abstract}

This study was conducted to provide a better understanding of the role of electric field strength in the production of aftereffects in resting state scalp electroencephalography by repetitive transcranial magnetic stimulation (rTMS) in humans. We conducted two separate experiments in which we applied rTMS over the left parietal-occipital region. Prospective electric field simulation guided the choice of the individual stimulation intensities. In the main experiment, 16 participants received rhythmic and arrhythmic rTMS bursts at between ca. 20 and $50 \mathrm{mv} / \mathrm{mm}$ peak absolute electric field intensities. In the control experiment, another group of 16 participants received sham rTMS. To characterize the aftereffects, we estimated the alpha power $(8-14 \mathrm{~Hz})$ changes recorded in the inter-burst intervals, i.e., from 0.2 to 10 seconds after rTMS. We found aftereffects lasting up to two seconds after stimulation with ca. $35 \mathrm{mv} / \mathrm{mm}$. Relative to baseline, alpha power was significantly reduced by the arrhythmic protocol, while there was no significant change with the rhythmic protocol. However, we found no significant long-term, i.e., up to 10-second, differences between the rhythmic and arrhythmic stimulation, or between the rhythmic and sham protocols. Weak arrhythmic rTMS induced short-lived alpha suppression during the inter-burst intervals. 


\subsection{Introduction}

The self-organized activity of neurons and neural assemblies produces oscillating electric fields in the brain ${ }^{175}$. These oscillating electric fields are recurrent, as they feed back onto the neural assemblies thereby facilitating neural synchrony and plasticity ${ }^{175}$. Repetitive transcranial magnetic stimulation (rTMS) induces a periodic electromagnetic field in the brain ${ }^{81}$, which triggers molecular, cellular, and electrophysiological changes in neuro-glia networks ${ }^{176}$.

In our previous work, we studied the immediate electrophysiological effects of rTMS using a novel stimulation intensity selection approach ${ }^{72}$. In order to individually adapt the stimulation intensities, we prospectively estimated the rTMS-induced electric field strengths ${ }^{72}$. Using this approach we have shown that peak absolute electric fields between ca. 35 and $50 \mathrm{mv} / \mathrm{mm}$ already induced immediate changes in the electroencephalogram (EEG) in humans ${ }^{72}$.

Yet, many applications of rTMS aim at inducing neural effects that outlast the duration of the stimulation itself. Therefore, in the present study we investigated possible aftereffects of the stimulation by focusing on the EEG recordings in the inter-burst intervals from 0.2 to $10 \mathrm{~s}$ after the rTMS bursts. The selected time window is free from rTMS-induced artifacts such as ringing, decay, cranial muscular, somatosensory or auditory artifacts ${ }^{177}$.

To quantify the aftereffects, we estimated the spectral power in the alpha frequency band which is a common outcome measure in the rTMS-EEG literature ${ }^{178}$. Based on the entrainment echo hypothesis ${ }^{179}$, we expected that rhythmic rTMS at the individual alpha frequencies would entrain neural oscillations and increase alpha power due to facilitated spike-timing dependent plasticity. On the other hand, we expected that arrhythmic (active control) or sham ( $90^{\circ}$ tilt) protocols would not entrain ongoing posterior alpha oscillation and, therefore, would not produce any aftereffects. 


\subsection{Methods}

\subsubsection{Secondary analysis}

To test our hypotheses we performed a secondary analysis of our openly available rTMSEEG dataset (https://github.com/ZsoltTuri/2019_rTMS-EEG). We reported the immediate electrophysiological effects elsewhere ${ }^{72}$. This dataset contains EEG recordings from two separate experiments (see point 3.3.5 for more details).

\subsubsection{Participants}

We included only neurologically healthy participants in the study ${ }^{72}$. For more details, see Table 4.

\begin{tabular}{lll}
\cline { 2 - 3 } & Main experiment & Control experiment \\
\hline Sample size (n) & 16 & 16 \\
Mean age \pm SD (years) & $25.5 \pm 3.2$ & $23.9 \pm 3.9$ \\
Age range (years) & 21 to 32 & 20 to 34 \\
Number of women/men & $8 / 8$ & $8 / 8$ \\
Exclusion criteria assessed by & Self-reports and/or neurological examinations \\
Contraindications & None & None \\
Mean laterality index ${ }^{\mathrm{a}} \pm \mathrm{SD}$ & $78.4 \pm 50.1$ & $78.8 \pm 31.6$ \\
Laterality index range & -30 to 100 & 0 to 100 \\
\hline
\end{tabular}

Table 4. Participant information. We assessed the handedness laterality index with the Edinburgh Handedness Inventory ${ }^{120}$. 


\subsubsection{Ethics}

The Ethics Committee of the University Medical Center Göttingen approved the investigation, the experimental protocols, and all methods used in the main and control experiment (application number: 35/7/17). We performed all the experiments under the relevant guidelines and regulations. All participants gave written informed consent before participation ${ }^{72}$.

\subsubsection{Head modeling and electric field estimation}

We used a freely available open software package called Simulation of Non-invasive Brain Stimulation (SimNIBS, version 2.0.1) ${ }^{122}$. We used anatomical T1- and T2-weighted and diffusionbased magnetic resonance imaging data (MRI) to generate individualized, multi-compartment head models. The head models included the following compartments (corresponding conductivity values in $[\mathrm{S} / \mathrm{m}])$ : scalp (0.465), bone (0.01), cerebrospinal fluid (1.654), gray matter $(0.275)$ and white matter (0.126). For the gray and white matter compartments, we used anisotropic conductivity values using the volume-normalized method ${ }^{124}$.

\subsubsection{Experimental procedure and stimulation parameters}

In the main experiment $(n=16)$, we performed prospective electric field modeling to individually adapt the stimulation intensities (see Ошибка! Источник ссылки не найден.2А). Participants took part in three rTMS-EEG sessions separated by at least 48 hours. In each session, we applied rTMS at 20,35, or $50 \mathrm{mV} / \mathrm{mm}$ peak absolute electric fields. These field values correspond to $9.5 \pm 1.1 \%, 16.8 \pm 2 \%$, and $23.9 \pm 2.5 \%$ of the group-averaged device output. We refer to these 
sessions as Low, Medium, and High intensity conditions, respectively. For further details about the rTMS protocols, see Ошибка! Источник ссылки не найден.2B (top).

In the control experiment $(\mathrm{n}=16)$, an independent group of participants received sham rTMS with the coil tilted by $90^{\circ}$ (see Ошибка! Источник ссылки не найден.2B, bottom) ${ }^{119}$. During the measurement, this sham protocol produced acoustic and ringing/decay artifacts while it minimized the induced electric field in the brain. We used the same stimulation intensity for each participant, which we fixed at $29 \%$ of the device output. This value corresponded to the maximum pulse amplitude used in the High intensity condition of the main experiment.

In both experiments, we applied rTMS over the left parietal-occipital area, specifically at the PO3 electrode as defined by the international 10/20 EEG system. The participants received the stimulation in the resting state, eyes open condition (Ошибка! Источник ссылки не найден.2C). We delivered the rhythmic rTMS at the individual alpha frequency, which we estimated prior to each session from the resting state EEG recordings ${ }^{72}$. Based on the Arnold's tongue model of neural entrainment, this is a necessary step to maximize the efficacy of inducing neural entrainment. In the arrhythmic rTMS, we applied rTMS in a manner that avoided any rhythmicity in the timing of the consecutive pulses ${ }^{86,180}$. Here, we prospectively adjusted the timing of each pulse so that frequencies in the alpha frequency band $(8-12 \mathrm{~Hz})$ as well as their harmonics and subharmonics did not occur (e.g., 4 and $16 \mathrm{~Hz}$ for $8 \mathrm{~Hz})^{72}$.

In both experiments, we used a MagPro X100 stimulator with MagOption (MagVenture, Denmark), normal coil current direction, biphasic pulses with $280 \mu$ s pulse width, and a MC-B70 figure-of-eight coil. During rTMS we simultaneously recorded the scalp EEG with a TMScompatible, 64 channel, active EEG system (BrainProducts, Munich, Germany). 


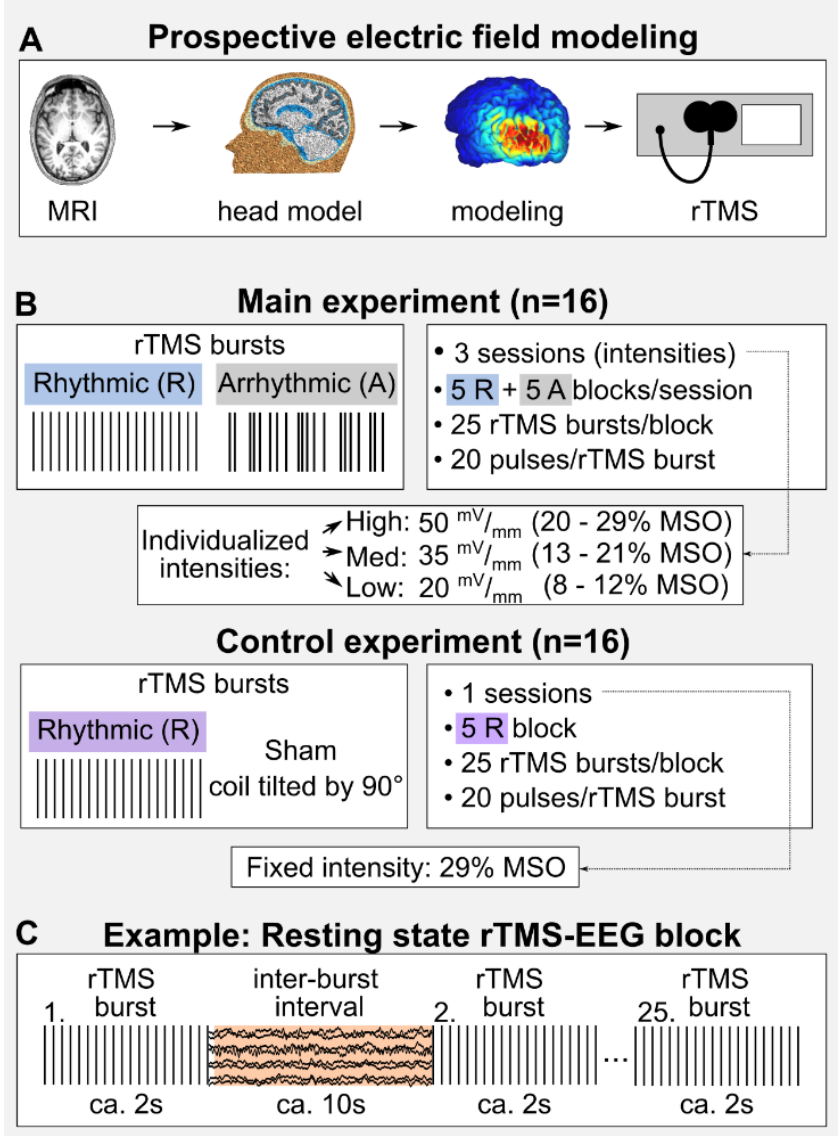

Figure 12. Study overview. (A) The stimulation intensity was individually adapted based on prospective electric field modeling. (B) The stimulation parameters in the main and control experiments. In the control experiment, we delivered rhythmic sham rTMS. (C) We defined the aftereffects by focusing on the rTMS artifact-free inter-burst intervals (highlighted in orange). Abbreviations: $M S O$ - maximum stimulator output.

\subsubsection{EEG analysis}

EEG preprocessing. EEG analysis was performed using the FieldTrip software package (http://fieldtrip.fcdonders.nl) with custom-made MATLAB code. First, the TMS-EEG data were segmented into trials that were time-locked to the offset of the rTMS burst (from 3.5s before and $10 \mathrm{~s}$ after the last TMS pulse). The datasets in both experiments (main and control) included 125 
trials with each stimulation condition. We removed the rTMS-induced ringing artifacts from $4 \mathrm{~ms}$ before to $9 \mathrm{~ms}$ after the TMS pulse. The first round of ICA (fastICA) was performed to automatically identify the decay artifact by averaging the time course of components over $50 \mathrm{~ms}$ after each TMS pulse. Components with an amplitude exceeding $30 \mu \mathrm{V}$ were rejected. Piecewise Cubic Hermite Interpolation (pchip) replaced the time intervals around the pulses.

Then, the data were downsampled to $625 \mathrm{~Hz}$. We applied a $80 \mathrm{~Hz}$ low-pass and a $0.1 \mathrm{~Hz}$ high-pass filter (Butterworth IIR filter type, 'but' in FieldTrip). A discrete Fourier transform-based filter was used to remove the $50 \mathrm{~Hz}$ line noise. Next, the data were inspected for artifactual trials and channels. The procedure included a semi-automatic algorithm described in detail in reference 95. In brief, we defined the outlier channels and trials, which exceeded 1.5 interquartile ranges. If a trial contained fewer than $20 \%$ of such channels, they were interpolated in the trial, but otherwise removed. The channels with line noise or high impedance levels were defined by estimating the correlation coefficient with the neighboring channels. We rejected channels that had a correlation coefficient value lower than 0.4 with their neighbors. All removed channels were then interpolated using the weighted signal of the neighboring channels.

After inspecting the data we defined the number of independent components for the ICA (binICA) by estimating the eigenvalues of the covariance matrix of the EEG data. We defined the number of ICA components as the rank of the diagonal matrix minus the number of the interpolated channels. We ran ICA only on trials that did not contain any interpolated channels. Independent components were visually inspected for artifacts. The components containing eye-related artifacts, muscle, and line noise artifacts were projected out from the data. After preprocessing, 93.8 \pm 9.9 (mean \pm SD) trials remained for the High, 91.1 \pm 13.4 trials for the Medium and $92.5 \pm 9.9$ trials for 
the Low-intensity conditions. As the last preprocessing step, we applied two seconds of padding ('mirror') to the data intervals corresponding to baseline.

Short-term aftereffect. We performed the time-frequency analysis by running Wavelet decomposition on frequencies from 1 to $25 \mathrm{~Hz}$ for the whole length of the trial from -5.5 to 10 seconds around the TMS burst offset. The wavelet consisted of seven cycles with 3 Gaussian widths. Once the wavelet analysis was completed, we performed a statistical analysis to test the short-term aftereffect of the protocols and the time. To this aim, we used two-second intervals before ('baseline') and after ('activation') the rTMS burst. For each participant we averaged the data over all trials and then performed the statistical analysis (Fieldtrip as 'actvsbslT' test) separately for each intensity condition (High, Medium, and Low). To reduce the influence of the remaining TMS artifacts we performed a cluster-based permutation test (Monte Carlo, 2-25 Hz frequency range two-tailed t-test with 1,000 permutations) $0.2 \mathrm{~s}$ after the last TMS pulse. The null hypothesis was rejected if the p-value of the maximum cluster level statistics was below 0.05 (onetailed test).

Long-term after effect. For the second analysis, we normalized the power of all intervals of ca. 10 seconds length after rTMS bursts to baseline, i.e., the 1s period before the start of the rTMS burst, using the decibel conversion. The frequency range was normalized by extracting the IAF from the original frequency, and was averaged over IAF $\pm 1 \mathrm{~Hz}$ and over the ten left parietal channels (i.e., P7, P5, P3, P1, Pz, PO7, PO3, POz, O1, Oz).

Statistical analysis of the normalized power including ten channels and the entire trial duration from zero to ten seconds was performed for each stimulation intensity separately. First, we used the independent samples t-test to compare rhythmic real and rhythmic sham rTMS protocols in the High-intensity condition. When comparing the real and sham rhythmic protocols, 
we focused primarily on the high intensity condition because our participants received only one sham rTMS session corresponding to the high intensity condition in the main experiment. Note that in the sham protocol we fixed the stimulation intensity at $29 \%$ of the device output. To compare the rhythmic and arrhythmic conditions we used dependent sample t-tests separately for each intensity condition at IAF $\pm 1 \mathrm{~Hz}$. A non-parametric Monte Carlo approach with 1,000 randomizations was performed to estimate the probability of whether a given amount of significant electrodes $(p<0.05)$ could be expected by chance.

\subsection{Results}

\subsubsection{Short-term aftereffect}

First, we focused on analyzing the alpha power change following the rTMS bursts and compared it to the baseline value. In the rhythmic conditions, the analysis revealed no statistically significant differences from baseline in any of the intensity conditions (see Fig. 13). Note that in the Medium intensity condition the change was nearly significant $(\mathrm{p}=0.07)$. However, in the arrhythmic conditions there was a significant change with the Medium intensity $(p=0.03)$, but not with any other intensity (see Fig. 13B). Lastly, the analysis revealed that the alpha power did not change significantly from baseline after the sham protocol (Fig. 13C). Note that the present study used only one sham condition as a control for the High intensity rhythmic condition. 


\section{A Rhythmic}
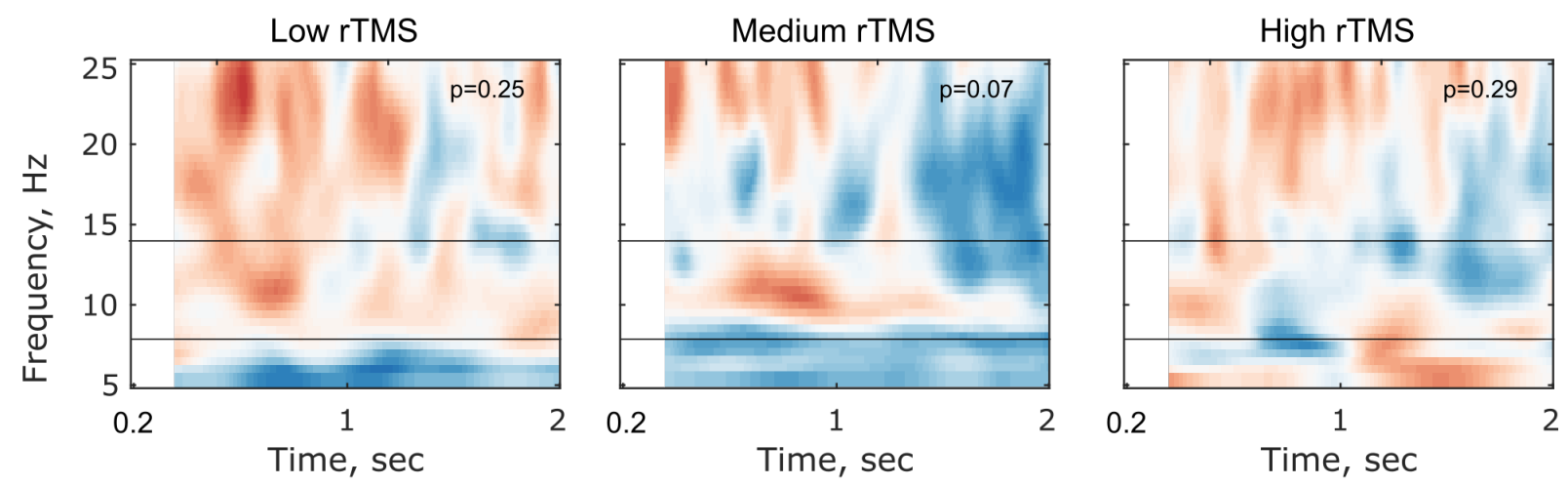

\section{B Arrhythmic}
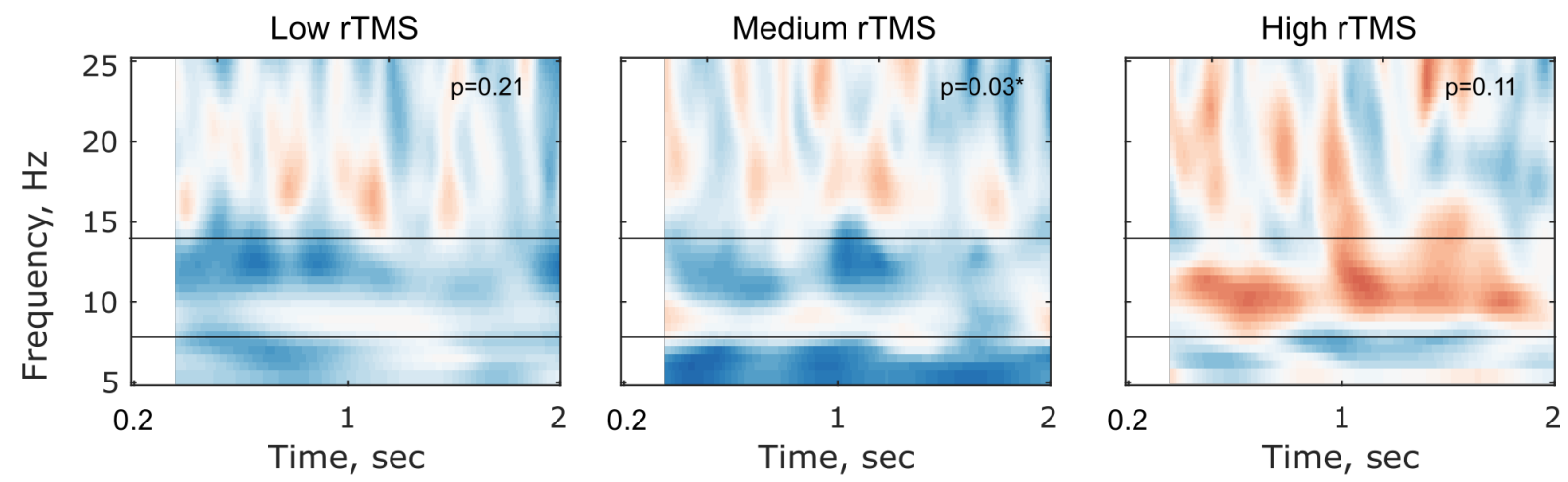

\section{Rhythmic sham}

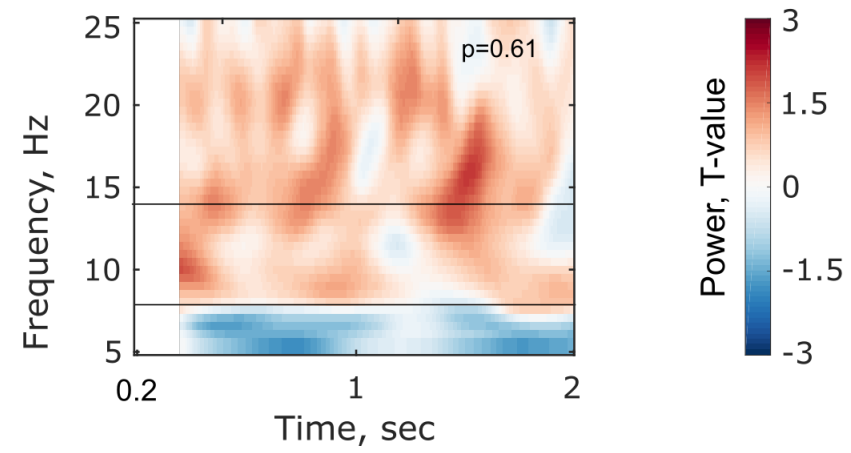

Figure 13. Alpha power change after the rTMS bursts compared with the baseline time period (activation vs. baseline analysis). Time-frequency plots show the power in the range from 5 to 25 $\mathrm{Hz}(A)$ in the rhythmic, main, $(B)$ in the arrhythmic, control and $(C)$ in the sham rTMS protocols. Horizontal lines represent the limits of alpha rhythm (8-14 Hz). Zero on the abscissa corresponds 
to the time of stimulation offset. Statistical analysis was performed with a gap of $200 \mathrm{~ms}$ to reduce the influence of residual TMS artifacts.

\subsubsection{Long-term aftereffect}

In the following analyses, we focused on the IAF, because the entrainment hypothesis predicts that the most pronounced effects should occur in frequencies at and close to the IAF ${ }^{92}$. We compared the rhythmic and sham protocols in the High intensity condition using a nonparametric cluster-based permutation test of the normalized alpha power. The analysis did not reveal any significant difference between the real and sham groups ( $p=0.30$; Ошибка! Источник ссылки не найден. 14).

High rTMS

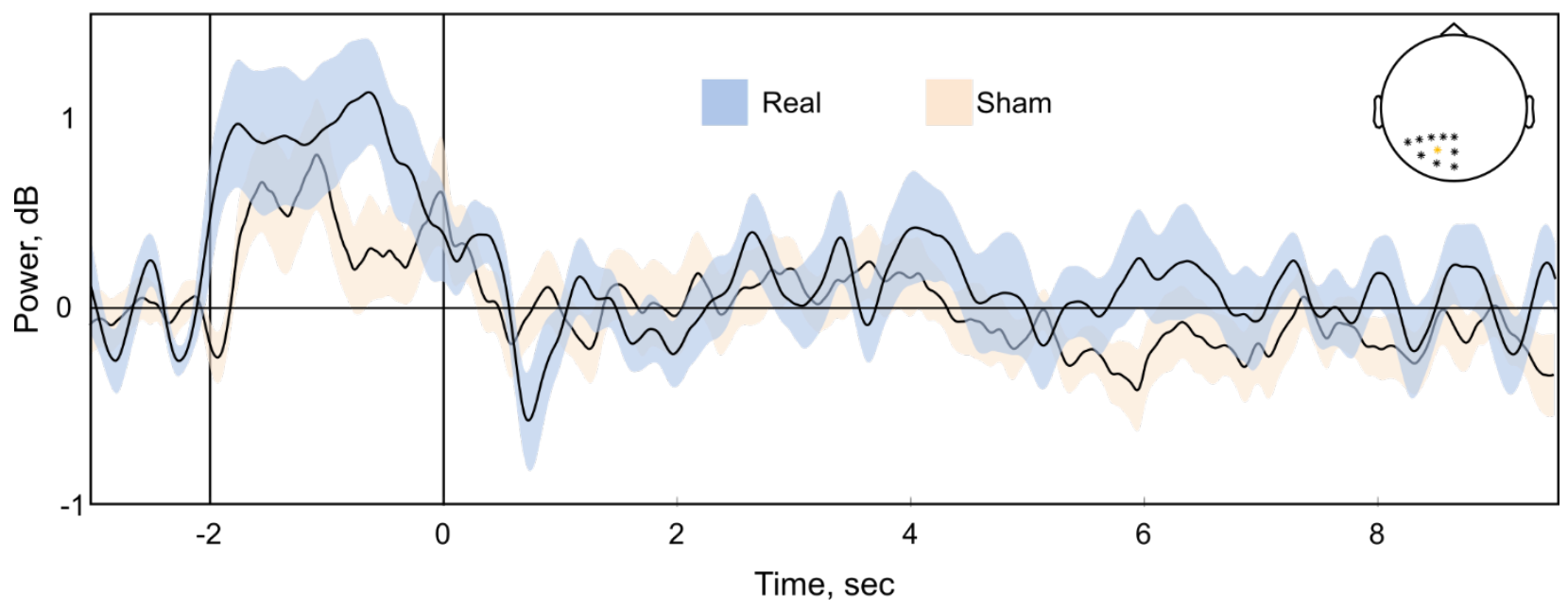

Figure 14. Real rTMS did not change the spectral power relative to the sham rTMS at the individual alpha frequency. The plots show the mean (black line) and SEM (shaded area) of normalized alpha power during the whole trial. The power at IAF $\pm 1 H z$ was averaged over ten parietal channels around the stimulation electrode - PO3 (red). The vertical lines at -2 and zero seconds represent 
stimulation onset and offset, respectively. Note that we aligned the analysis relative to the end of rTMS bursts. Thus, the exact beginning at -2 second varies according to the IAF.

Next, we compared the rhythmic and arrhythmic protocols using non-parametric clusterbased permutation tests on the normalized alpha power. Again, the test revealed no significant differences between these protocols either in the High $(p=0.18)$, Medium $(p=0.08)$, or Low $(p=$ 0.23 ) intensity conditions (see Fig. 15). 


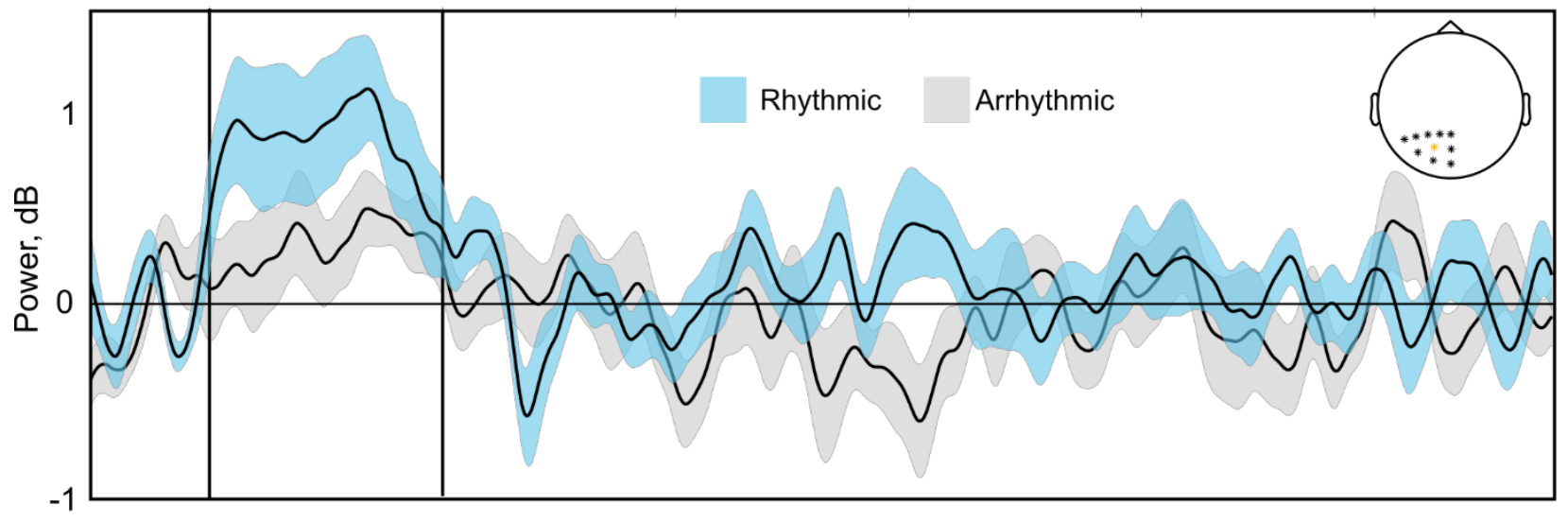

\section{Medium rTMS}

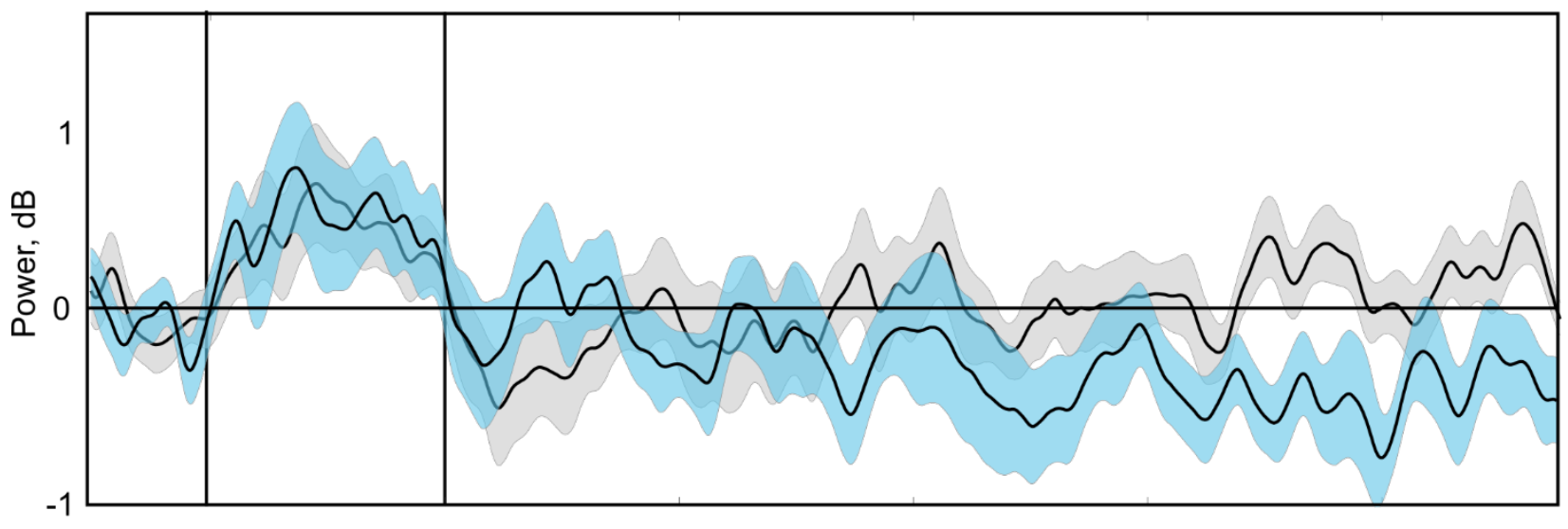

\section{Low rTMS}

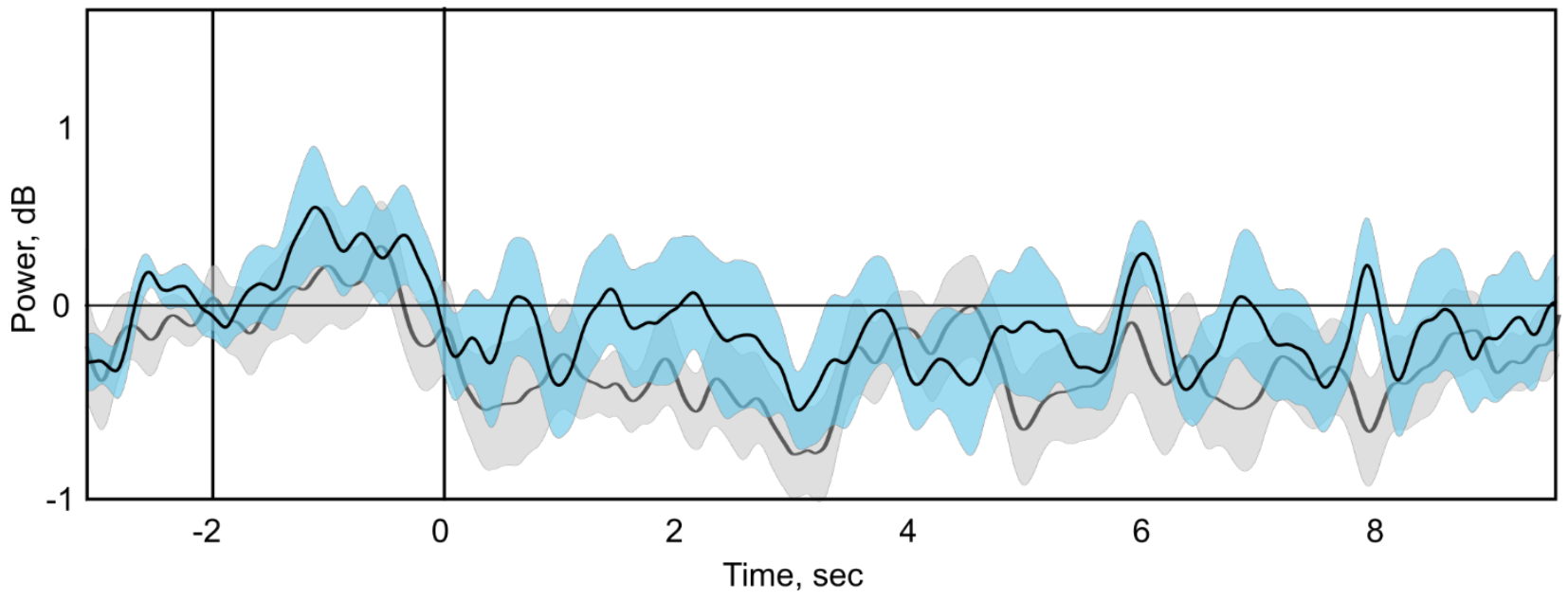

Figure 15. Lack of significant differences in the individual alpha power between rhythmic and arrhythmic rTMS. The plots show the mean (black line) and SEM (shaded area) of alpha power after rTMS bursts (time $=0$ ). The power is normalized to the 1-second-long baseline period directly 
before the rTMS bursts with decibel correction and averaged over groups and ten parietal channels. Alpha power is extracted at IAF $\pm 1 \mathrm{~Hz}$. Statistical analysis showed no significant difference between the rhythmic and arrhythmic conditions for any stimulation intensity. The vertical lines at -2 and zero seconds represent stimulation onset and offset, respectively. Note that we aligned the analysis relative to the end of rTMS bursts. Thus, the exact beginning at -2 second varies according to the IAF.

These findings indicate that relative to the arrhythmic, control conditions, real rTMS at ca. 20 and $50 \mathrm{mV} / \mathrm{mm}$ peak absolute electric field did not change the spectral power in the inter-burst intervals in the individual alpha frequency $\pm 1 \mathrm{~Hz}$ range. There was a non-significant $(\mathrm{p}=0.08)$

decrease in alpha power relative to the arrhythmic condition, real rTMS at ca. $35 \mathrm{mV} / \mathrm{mm}$ for up to 10 seconds.

\subsection{Discussion}

In the present study, we investigated the electrophysiological aftereffects of rhythmic, arrhythmic, and sham rTMS protocols in humans. We defined aftereffects as changes in the alpha power $(8-14 \mathrm{~Hz})$ during the inter-burst intervals. We measured short-term aftereffects, i.e. up to two seconds after stimulation, and long-term aftereffects, i.e. from two to ten seconds after stimulation. We expected that rhythmic rTMS would entrain alpha oscillations and lead to increased alpha power after rTMS ${ }^{179}$. Based on the entrainment echo hypothesis, we expected alpha power to be increased for up to ca. two seconds after each burst with rhythmic stimulation. We also expected that neither sham nor arrhythmic rTMS would have any aftereffects on power modulation.

Contrary to our expectations, we observed no aftereffects on alpha power in the rhythmic rTMS protocols with all intensities. In the medium intensity condition, we observed a significant 
decrease in alpha power in the arrhythmic, and a slight, but non-significant increase in the rhythmic protocol. When studying the entire ten-second inter-burst interval, we found no significant differences in alpha power between the rhythmic and sham or rhythmic and arrhythmic protocols.

Compared to conventional rTMS studies that typically use electric fields of ca. $100 \mathrm{mv} / \mathrm{mm}$, the present study applied field strengths that were several times weaker ranging from 20 to 50 $\mathrm{mV} / \mathrm{mm}$. One might argue that the applied electric field strength was simply too weak to induce any aftereffects. Following the above argument, one should find more robust aftereffects on alpha power in studies using much stronger stimulation intensities and thus greater electric field strengths. To gain a comprehensive overview, we performed a systematic literature search on rTMS studies using conventional intensities published between 1989 and 2017 (see S1 Appendix for details).

In this search, we focused on studies that evaluated the aftereffects of $10 \mathrm{~Hz}$ rTMS on alpha power. We identified 16 eligible articles; ten of which described no aftereffects after rTMS. Two articles described an increase, two articles observed both an increase and a decrease, and one article described a decrease. One article reported incomplete statistical tests to support the claimed aftereffect (e.g., post-hoc tests were missing; see Table 5 for more details). One plausible reason for the contradictory findings may be the known variability in the stimulation parameters, such as the number of pulses, duration of the inter-train intervals, the neuronal state of the stimulated area, etc. $^{109}$.

Moreover, these studies also differ in how they operationalize the rTMS-induced aftereffects. Whereas some studies focused on the short inter-burst intervals ${ }^{\text {e.g., }}{ }^{181}$, others analyzed the time interval after the end of the rTMS protocol ${ }^{\text {e.g., } 182}$. Furthermore, studies may also differ in whether they evaluate the aftereffects directly after the end of the rTMS protocol or after a certain delay 
period $^{\text {e.g., } 61}$. In the present literature search, this delay period varied from several minutes ${ }^{\text {e.g., } 183}$ up to one week e.g., 184 . Finally, these studies recruited healthy persons as well as patients (e.g., medication resistant major depression ${ }^{183}$ ), which is an important factor to consider when evaluating the aftereffects of rTMS.

Taken together, it is difficult to draw comprehensive conclusions about the expected direction of the EEG aftereffects following $10 \mathrm{~Hz}$ rTMS. Therefore, the result of the literature analysis was that the evidence about the aftereffects on spectral power in conventional rTMS studies is currently inconclusive.

At conventional intensities, $10 \mathrm{~Hz}$ rTMS is supposed to increase the corticospinal excitability level ${ }^{109}$. The most typical outcome measure in humans is the peak-to-peak amplitude of the single pulse TMS-induced motor evoked potential. Many studies have found increased motor evoked potential amplitudes after the end of a $10 \mathrm{~Hz}$ rTMS protocol that lasted for a few minutes ${ }^{185}$. Inhibitory synaptic effects likely play a significant role in the pattern of aftereffects. For instance, a previous in vitro tissue culture study provided evidence that $10 \mathrm{~Hz}$ repetitive magnetic stimulation induced long-term potentiation in inhibitory synapses ${ }^{116}$. Moreover, scalp EEG alpha oscillations have been associated with cortical inhibition in humans ${ }^{117}$. Therefore, future studies should also investigate the aftereffects of $10 \mathrm{~Hz}$ rTMS on the corticospinal excitability level together with the EEG changes when applying weak electric fields, such as in the present study.

In the present study, we focused on electrophysiological aftereffect recorded during the interburst intervals. At medium intensities (ca. $35 \mathrm{mV} / \mathrm{mm}$ ), arrhythmic rTMS significantly reduced the alpha power shortly after the rTMS bursts, while the increase in alpha power after rhythmic rTMS was not statistically significant. These findings may be explained by previous observations that cortical inhibitory mechanisms might have lower intensity thresholds than those producing 
excitation ${ }^{115}$. It remains to be seen which electric field intensities can induce more robust and longterm aftereffects that are manifest for up to several minutes or even longer after the end of the protocol.

\subsection{Supplemental information}

We found 194 articles between January 2009 and December 2017 that described studies using rTMS at the alpha frequency band in humans. We selected studies delivering rTMS at $10 \mathrm{~Hz}$ and at individualized frequencies at alpha or mu rhythms. We excluded 145 articles that did not use the EEG to evaluate the effects of rTMS. We removed six articles that sequentially combined $1 \mathrm{~Hz}$ rTMS with $10 \mathrm{~Hz}$ rTMS as well as two prospective clinical trials. We identified 41 articles that combined rTMS with EEG measurements, 17 of which evaluated the effects of rTMS by assessing spectral power. We further excluded four articles that focused on immediate electrophysiological effects. Ten of the remaining thirteen articles used a fixed $10 \mathrm{~Hz}$ stimulation frequency. Two articles set the stimulation frequency at the individual mu rhythm, and one at the individual alpha rhythm (see Part I in Table 5).

We further divided the 13 articles based on the time period in which they analyzed the rTMSinduced electrophysiological aftereffects. High-frequency rTMS $(\geq 5 \mathrm{~Hz})$ protocols deliver the stimulation in short bursts/trains and therefore employ several seconds of inter-train intervals between each burst. For example, one can deliver 1,000 rTMS pulses in 20 bursts, using 50 pulses in each burst and $25 \mathrm{~s}$ inter-train intervals. The role of the inter-train interval is at least twofold: they prevent coil overheating, and are important for patient safety. Without inter-train intervals, the likelihood increases that high-frequency rTMS might induce an epileptic seizure even in 
healthy individuals. The short inter-train interval also allows recording and analyzing simultaneous scalp EEG periods that are free of rTMS-induced artifacts. Therefore, the EEG analysis can focus on these short inter-train intervals. It can start directly after the last pulse or several minutes after the end of the protocol. We identified four articles that analyzed the aftereffects during the intertrain intervals. Six articles focused on aftereffects occurring directly after the last pulse and four after the end of the stimulation protocol. This latter period varied between several minutes to one week. 
Table 5. Summary of studies investigating the rTMS-induced electrophysiological aftereffects.

Abbreviations: act: active/real stimulation; DLPFC: dorso-lateral prefrontal cortex; EEG RoT: EEG electrode landmark and rule of thumb; IAF: individual alpha frequency; IMF: individual mu frequency; IPL: inferior parietal lobe; iIPS: inferior intraparietal sulcus; ITI: intertrain intervals; MT: motor threshold; NN anat: neuronavigation based on individual anatomy; n: sample size; No.: number; n.s.: not significant; PT: phosphene threshold; RMD: repeated measures design; RMT: resting motor threshold; RoT: rule of thumb; RT: participants' reaction time; S1: primary somatosensory cortex; SD: single design without sham or control rTMS; sh: sham stimulation; SGD: separate group design; SPL: superior parietal lobule; sp-TMS: single pulse TMS; vMT: visual motor threshold.

\begin{tabular}{|c|c|c|c|c|c|c|c|c|c|c|c|c|c|c|c|c|c|}
\hline \multicolumn{18}{|c|}{ Part I. Our systematic literature search results } \\
\hline Nr. & Article & Sample & n & Dose & Target & $\begin{array}{c}\text { Target } \\
\text { selection }\end{array}$ & Frequency & $\begin{array}{l}\text { Total no. } \\
\text { of pulses }\end{array}$ & $\begin{array}{c}\text { Pulses/ } \\
\text { train }\end{array}$ & $\begin{array}{c}\text { Train } \\
\text { number }\end{array}$ & $\begin{array}{c}\text { Inter- } \\
\text { train } \\
\text { interval }\end{array}$ & $\begin{array}{l}\text { Session } \\
\text { number }\end{array}$ & $\begin{array}{l}\text { Sham/ } \\
\text { control } \\
\text { rTMS }\end{array}$ & Design & $\begin{array}{l}\text { rTMS } \\
\text { during }\end{array}$ & $\begin{array}{c}\text { EEG } \\
\text { period }\end{array}$ & Aftereffect on alpha power \\
\hline 1 & $\begin{array}{c}\text { Narushima } \\
2010\end{array}$ & $\begin{array}{l}\text { medication } \\
\text { resistant } \\
\text { vascular } \\
\text { depression } \\
\end{array}$ & $\begin{array}{l}\text { act: } \\
43 \\
\text { sh: } \\
22\end{array}$ & $\begin{array}{l}110 \% \\
\text { RMT }\end{array}$ & $\begin{array}{c}\text { left } \\
\text { DLPFC }\end{array}$ & $\begin{array}{l}\mathrm{NN} \\
\text { anat }\end{array}$ & 10 & 1,200 & 60 & 20 & 60 & $10 / 15$ & $\begin{array}{l}\text { sham } \\
\text { coil }\end{array}$ & SGD & rest & after & n.s. \\
\hline 2 & $\begin{array}{c}\text { Azila Noh } \\
2011\end{array}$ & healthy & 12 & $\begin{array}{l}100 \% \\
\text { RMT }\end{array}$ & $\begin{array}{c}\text { left } \\
\text { motor } \\
\text { cortex }\end{array}$ & $\begin{array}{l}\text { sp-TMS } \\
\text { hot spot }\end{array}$ & IMF & $400 / 1,200$ & 20 & $20 / 60$ & 68 & 1 & $\begin{array}{l}\text { tilt by } \\
90^{\circ}\end{array}$ & $\begin{array}{c}\text { RMD- } \\
\text { same } \\
\text { day }\end{array}$ & rest & ITI & increase \\
\hline 3 & $\begin{array}{c}\text { Valiulis } \\
2012\end{array}$ & $\begin{array}{l}\text { medication } \\
\text { resistant } \\
\text { major } \\
\text { depression }\end{array}$ & 23 & $\begin{array}{l}100 \% \\
\text { MT }\end{array}$ & $\begin{array}{c}\text { left } \\
\text { DLPFC }\end{array}$ & $\begin{array}{c}\text { sp-TMS } \\
\text { hot spot } \\
\text { RoT }\end{array}$ & 10 & 1,600 & 80 & 20 & 40 & $10 / 15$ & no & SD & rest & after & increase \\
\hline 4 & $\begin{array}{c}\text { Fuggetta } \\
2013\end{array}$ & healthy & $\begin{array}{c}\text { act: } \\
11 \\
\text { sh: } \\
11\end{array}$ & $\begin{array}{l}100 \% \\
\text { RMT }\end{array}$ & $\begin{array}{l}\text { left } \\
\text { motor } \\
\text { cortex }\end{array}$ & $\begin{array}{l}\text { sp-TMS } \\
\text { hot spot }\end{array}$ & 10 & 400 & 20 & 20 & 30 & 1 & $\begin{array}{l}\text { tilt by } \\
90^{\circ}\end{array}$ & SGD & rest & ITI & n.s. \\
\hline 5 & $\begin{array}{c}\text { Puzzo } \\
2013 \\
\end{array}$ & healthy & 14 & $\begin{array}{l}110 \% \\
\text { RMT }\end{array}$ & $\begin{array}{l}\text { left } \\
\text { IPL }\end{array}$ & $\begin{array}{c}\text { EEG } \\
\text { P3 }\end{array}$ & $\mathrm{IMF}+1$ & 720 & 24 & 30 & 15.6 & 1 & $\begin{array}{c}\text { no } \\
\text { rTMS }\end{array}$ & SD & task & ITI & n.s. \\
\hline 6 & $\begin{array}{l}\text { Pripfl } \\
2014\end{array}$ & SUD-nic & 11 & $\begin{array}{l}90 \% \\
\text { RMT }\end{array}$ & $\begin{array}{c}\text { left } \\
\text { DLPFC }\end{array}$ & $\mathrm{NN}$-anat & 10 & 1,200 & 50 & 24 & 25 & 1 & vertex & RMD & rest & $\begin{array}{l}\text { after } \\
\text { last } \\
\text { pulse }\end{array}$ & decrease \\
\hline 7 & $\begin{array}{l}\text { Weisz } \\
2014\end{array}$ & healthy & $\begin{array}{l}\text { act: } \\
15 \\
\text { sh: } \\
15\end{array}$ & $\begin{array}{l}50 \% \\
\mathrm{MSO}\end{array}$ & $\begin{array}{c}\text { left } \\
\text { auditory } \\
\text { cortex }\end{array}$ & $\begin{array}{l}\text { EEG } \\
\text { RoT }\end{array}$ & IAF & 1,000 & 50 & 20 & 25 & 1 & $\begin{array}{l}\text { tilt by } \\
45^{\circ}\end{array}$ & SGD & rest & $\begin{array}{l}\text { ITI \& } \\
\text { after } \\
\text { last } \\
\text { pulse }\end{array}$ & $\begin{array}{l}\text { ITI: Increase in real rTMS driven } \\
\text { by decrease in sham. } \\
\text { after: n.s. }\end{array}$ \\
\hline
\end{tabular}




\begin{tabular}{|c|c|c|c|c|c|c|c|c|c|c|c|c|c|c|c|c|c|}
\hline Nr. & Article & Sample & $\mathbf{n}$ & Dose & Target & $\begin{array}{c}\text { Target } \\
\text { selection }\end{array}$ & Frequency & $\begin{array}{l}\text { Total no. } \\
\text { of pulses }\end{array}$ & $\begin{array}{c}\text { Pulses/ } \\
\text { train }\end{array}$ & $\begin{array}{c}\text { Train } \\
\text { number }\end{array}$ & $\begin{array}{c}\text { Inter- } \\
\text { train } \\
\text { interval }\end{array}$ & $\begin{array}{c}\text { Session } \\
\text { number }\end{array}$ & $\begin{array}{l}\text { Sham/ } \\
\text { control } \\
\text { rTMS }\end{array}$ & Design & $\begin{array}{l}\text { rTMS } \\
\text { during }\end{array}$ & $\begin{array}{c}\text { EEG } \\
\text { period }\end{array}$ & Aftereffect on alpha power \\
\hline 8 & $\begin{array}{c}\text { Wozniak- } \\
\text { Kwasniewska } \\
2014\end{array}$ & healthy & 20 & $\begin{array}{l}120 \% \\
\text { RMT }\end{array}$ & $\begin{array}{c}\text { left } \\
\text { DLPFC }\end{array}$ & $\begin{array}{l}\mathrm{NN} \\
\text { anat }\end{array}$ & 10 & 800 & 50 & 16 & 54 & 1 & $\begin{array}{c}\text { sham } \\
\text { coil }\end{array}$ & RMD & rest & $\begin{array}{l}\text { after } \\
\text { last } \\
\text { pulse }\end{array}$ & n.s. \\
\hline 9 & $\begin{array}{l}\text { De Felice } \\
2016\end{array}$ & SUD-alc & $\begin{array}{c}\text { act: } \\
10 \\
\text { sh: } \\
10\end{array}$ & $\begin{array}{l}100 \% \\
\text { RMT }\end{array}$ & $\begin{array}{c}\text { left } \\
\text { DLPFC }\end{array}$ & $\begin{array}{c}\text { EEG } \\
\text { F3 }\end{array}$ & 10 & 1,000 & 50 & 20 & 20 & 4 & $\begin{array}{l}3 \mathrm{~cm} \\
\text { wooden } \\
\text { plate }\end{array}$ & SGD & rest & after & n.s. \\
\hline 10 & $\begin{array}{l}\text { Kim } \\
2016\end{array}$ & healthy & $\begin{array}{c}\text { act: } \\
12 \\
\text { sh: } \\
12\end{array}$ & $\begin{array}{l}110 \% \\
\text { vMT }\end{array}$ & $\begin{array}{c}\text { left } \\
\text { DLPFC }\end{array}$ & $\begin{array}{c}\text { sp-TMS } \\
\text { hot spot } \\
\text { RoT }\end{array}$ & 10 & 1,600 & 50 & 32 & $\mathrm{n} / \mathrm{r}$ & 12 & $\begin{array}{c}\text { tilt by } \\
90^{\circ}\end{array}$ & SGD & rest & $\begin{array}{l}\text { after } \\
\text { last } \\
\text { pulse }\end{array}$ & $\begin{array}{l}\text { increase } \\
\text { decrease }\end{array}$ \\
\hline 11 & $\begin{array}{l}\text { Pathak } \\
2016 \\
\end{array}$ & $\begin{array}{c}\text { major } \\
\text { depression }\end{array}$ & 5 & $\begin{array}{l}100 \% \\
\text { MT }\end{array}$ & $\begin{array}{c}\text { left } \\
\text { DLPFC }\end{array}$ & $\begin{array}{l}\mathrm{NN} \\
\text { anat }\end{array}$ & 10 & 1,000 & 50 & 20 & 20 & 20 & no & SD & rest & after & n.s. \\
\hline 12 & $\begin{array}{c}\text { Möbius } \\
2017\end{array}$ & healthy & 23 & $\begin{array}{l}110 \% \\
\mathrm{MT}\end{array}$ & $\begin{array}{c}\text { left } \\
\text { DLPFC }\end{array}$ & $\begin{array}{c}\text { EEG } \\
\text { F3 }\end{array}$ & 10 & 3,000 & 50 & 60 & 25 & 1 & $\begin{array}{c}\text { tilt by } \\
45^{\circ}\end{array}$ & RMD & rest & $\begin{array}{c}\text { after } \\
\text { last } \\
\text { pulse }\end{array}$ & n.s. \\
\hline 13 & $\begin{array}{c}\text { Xia } \\
2017\end{array}$ & DOC & $\begin{array}{l}18 \\
12\end{array}$ & $\begin{array}{l}90 \% \\
\text { RMT }\end{array}$ & $\begin{array}{c}\text { left } \\
\text { DLPFC }\end{array}$ & $\begin{array}{c}\text { EEG } \\
\text { F3 }\end{array}$ & 10 & 1,000 & 100 & 10 & 60 & $\begin{array}{c}1 \\
20\end{array}$ & no & $\mathrm{SD}$ & rest & $\begin{array}{l}\text { after } \\
\text { last } \\
\text { pulse }\end{array}$ & n.s. \\
\hline
\end{tabular}

Part II. Selected studies from a review of Thut and Pascual-Leone (2010)

\begin{tabular}{|c|c|c|c|c|c|c|c|c|c|c|c|c|c|c|c|c|c|}
\hline Nr. & Article & Sample & $\mathbf{n}$ & Dose & Target & $\begin{array}{c}\text { Target } \\
\text { selection }\end{array}$ & Frequency & $\begin{array}{l}\text { Total no. } \\
\text { of pulses }\end{array}$ & $\begin{array}{c}\text { Pulses/ } \\
\text { train }\end{array}$ & $\begin{array}{c}\text { Train } \\
\text { number }\end{array}$ & $\begin{array}{c}\text { Inter- } \\
\text { train } \\
\text { interval }\end{array}$ & $\begin{array}{c}\text { Session } \\
\text { number }\end{array}$ & $\begin{array}{c}\text { Sham/ } \\
\text { control } \\
\text { rTMS }\end{array}$ & Design & $\begin{array}{l}\text { rTMS } \\
\text { during }\end{array}$ & $\begin{array}{c}\text { EEG } \\
\text { period }\end{array}$ & Aftereffect on alpha power \\
\hline 14 & $\begin{array}{c}\text { Okamura } \\
2001\end{array}$ & healthy & $\begin{array}{c}\text { act: } \\
20 \\
\text { sh: } \\
12\end{array}$ & $\begin{array}{c}100 \% \\
\text { MT }\end{array}$ & $\begin{array}{l}\text { left } \\
\text { PFC }\end{array}$ & RoT & 10 & 60 & 30 & 2 & 300 & 1 & $\begin{array}{c}\text { tilt by } \\
90^{\circ}\end{array}$ & SGD & rest & $\begin{array}{c}\text { after } \\
\text { last } \\
\text { pulse }\end{array}$ & no statistical test reported \\
\hline 15 & $\begin{array}{c}\text { Klimesch } \\
2003\end{array}$ & healthy & 15 & $\begin{array}{l}110 \% \\
\text { RMT }\end{array}$ & $\begin{array}{l}\text { right } \\
\text { IPS } \\
\text { mid } \\
\text { frontal }\end{array}$ & $\begin{array}{c}\text { EEG } \\
\text { P6 } \\
\text { Fz }\end{array}$ & $\mathrm{IAF}+1$ & 1,728 & 24 & 72 & $\begin{array}{c}11.6+ \\
\text { RT }\end{array}$ & 1 & $\begin{array}{c}\text { tilt by } \\
90^{\circ} \\
\text { IAF-3 } \\
20 \mathrm{~Hz}\end{array}$ & RMD & task & ITI & $\begin{array}{l}\text { decrease } \\
\text { increase }\end{array}$ \\
\hline 16 & $\begin{array}{c}\text { Griskova } \\
2007\end{array}$ & healthy & 18 & $\begin{array}{c}\text { act: } \\
110 \% \\
\text { RMT } \\
\text { sh: } \\
90 \% \\
\text { RMT }\end{array}$ & $\begin{array}{c}\text { left } \\
\text { DLPFC }\end{array}$ & $\begin{array}{c}\text { EEG } \\
\text { F3 } \\
\text { RoT }\end{array}$ & 10 & 2,000 & 20 & 100 & 10 & 1 & $\begin{array}{c}\text { tilt by } \\
45^{\circ}\end{array}$ & RMD & rest & $\begin{array}{c}\text { after } \\
\text { last } \\
\text { pulse }\end{array}$ & n.s. \\
\hline
\end{tabular}




\section{Inhibiting corticospinal excitability by entraining ongoing $\mu$ -}

\section{alpha rhythm in motor cortex}

Elina Zmeykina ${ }^{1}$, Zsolt Turi ${ }^{2}$, Andrea Antal $^{1}$, Walter Paulus ${ }^{1}$

${ }^{1}$ Department of Clinical Neurophysiology, University Medical Center Göttingen, Göttingen, Germany

${ }^{2}$ Department of Neuroanatomy, Institute of Anatomy and Cell Biology, University of Freiburg, Germany

Journal of Neuroscience, under review

Preprint (Biorxiv DOI): https://doi.org/10.1101/2020.11.11.378117

\section{Author contributions:}

EZ: conceptualization, study design, project administration, investigation (literature search, data collection), software, formal analysis, visualization, data interpretation, data curation, and writing the original draft

ZT: conceptualization, methodology, formal analysis, visualization, data interpretation, and writing the original draft

AA: data interpretation, and writing the original draft

WP: conceptualization, study design, funding acquisition, resources, data interpretation, and writing the original draft

Copyright (C) 2020, The Author(s). This is an open access article distributed under the terms of the Creative Commons CC BY license, which permits unrestricted use, distribution, and reproduction in any medium, provided the original work is properly cited. 


\subsection{Abstracts}

Sensorimotor $\mu$-alpha rhythm reflects the state of cortical excitability. Repetitive transcranial magnetic stimulation (rTMS) can modulate neural synchrony by inducing periodic electric fields (EFs) in the cortical networks. We hypothesized that the increased synchronization of $\mu$-alpha rhythm would inhibit the corticospinal excitability reflected by decreased motor evoked potentials (MEP). In seventeen healthy participants, we applied rhythmic, arrhythmic, and sham rTMS over the left M1. The stimulation intensity was individually adapted to $35 \mathrm{mV} / \mathrm{mm}$ using prospective EF estimation. This intensity corresponded to ca. $40 \%$ of the resting motor threshold. We found that rhythmic rTMS increased synchronization of $\mu$-alpha rhythm, increased $\mu$-alpha/beta power, and reduced MEPs. On the other hand, arrhythmic rTMS did not change the ongoing $\mu$-alpha synchronization or MEPs, though it increased the alpha/beta power. We concluded that low intensity, rhythmic rTMS can synchronize $\mu$-alpha rhythm and modulate the corticospinal excitability in M1. 


\subsection{Introduction}

Synchronously fluctuating transmembrane currents generate rhythmic activity in neural ensembles. These current flow alterations go along with the rhythmic shift between higher and lower excitability states ${ }^{186}$.

Sensorimotor $\mu$-alpha power in the range of $8-13 \mathrm{~Hz}$ reflects the corticospinal excitability fluctuation in the motor cortex. In humans, $\mu$-alpha excitability can be measured non-invasively with the electro-/magnetoencephalogram (EEG/MEG) ${ }^{187}$, or by recording motor evoked potentials (MEP) after transcranial magnetic stimulation (TMS). The amplitude of the MEP depends on the timing of the TMS pulse relative to the phase of the sensorimotor $\mu$-alpha wave 188. When TMS is delivered at the trough of the $\mu$-alpha wave, the MEP amplitudes are larger than with TMS delivered randomly or at the peak ${ }^{188}$.

Increased synchronization of the $\mu$-alpha rhythm over central and parietal areas occurs during a motor inhibition task ${ }^{189}$. MEP amplitudes were reduced during the inhibition condition when participants had to inhibit the motor response as compared to the motor activation task and baseline ${ }^{189}$. These findings support the inhibition-timing hypothesis that assumes that $\mu$-alpha oscillation is induced by inhibitory cells and reflects the shifts between the phases of maximal and minimal inhibition states ${ }^{25}$.

Ten Hz rTMS tends to increase the corticospinal excitability in most participants ${ }^{190}$. This effect may appear opposite to the inhibition-timing hypothesis by which induced $\mu$-alpha rhythm should inhibit rather than increase the corticospinal excitability. However, not one single parameter defines the neural mechanism of rTMS but rather the combination of the frequency and amplitude of stimulation and duration of the protocols.

We propose that one crucial parameter in deciding the effects of rTMS on corticospinal excitability level is the degree of $\mu$-alpha synchronization. We predict that if $10 \mathrm{~Hz}$ rTMS can increase the degree of $\mu$-alpha synchronization in the motor cortex, it should shift the oscillatory 
state into inhibition and temporarily decrease the corticospinal excitability. On the other hand, a $10 \mathrm{~Hz}$ protocol might perturb, rather than increase the degree of $\mu$-alpha synchronization. This might be the case at high stimulation intensities or when using arrhythmic rTMS. By reducing $\mu$-alpha synchronization cortical inhibition would be less and thereby it would result in a temporarily increase of corticospinal excitability.

In our previous study ${ }^{72}$ we used a novel stimulation intensity selection approach for rTMS, which was based on prospective electric field (EF) estimation. We observed ongoing parietaloccipital alpha synchronization at comparably low intensities in the range of $30-42 \%$ of the resting motor threshold corresponding to 35 and $50 \mathrm{mV} / \mathrm{mm}^{72}$.

In the present study, we extended our previous study by focusing on effects and aftereffects on sensorimotor $\mu$-alpha synchronization and corticospinal excitability level. To this aim, we applied rhythmic, arrhythmic, and sham rTMS protocols over the left primary motor cortex (M1) at $35 \mathrm{mV} / \mathrm{mm}$ EF strength. We hypothesized that desynchronized $\mu$-alpha activity would reflect a state of comparatively high excitability, whereas synchronized $\mu$-alpha activity would reflect a state of inhibition and low excitability. We predicted that rhythmic rTMS would increase local $\mu$-alpha synchronization and inhibit corticospinal excitability level, and hence reduce MEP amplitudes. On the other hand, we expected that arrhythmic rTMS would perturb $\mu$-alpha oscillations and thereby lead to motor cortex excitation, i.e. to, increased MEP amplitudes.

\subsection{Methods}

\subsubsection{Participants}

Seventeen neurologically healthy volunteers (eight females) participated in the study. The age range was $24-32$ years (mean \pm SD: $27.4 \pm 2.8$ years). Although the dataset of one participant was incomplete we included the data in the analysis. Two participants did not complete the 
experiments due to a high resting motor threshold and non-tolerability of TMS. The sample size was determined based on earlier rTMS-EEG studies ${ }^{191-193}$.

Before participation all volunteers filled out self-completed questionnaires to assess the study exclusion criteria. In cases of possible contraindications, a neurologist at the Department of Clinical Neurophysiology, University Medical Center Göttingen examined the volunteer. Inclusion criteria were no history or presence of medical, neurological, or psychiatric illnesses including epilepsy, drug and/or alcohol abuse, and no metal implants in the head, neck, or chest. We used the Edinburgh Handedness Inventory questionnaire to estimate the laterality index of participants. We included only right-handed participants with an index range of 30-100 (mean laterality index \pm SD: $75 \pm 15.5)$.

All participants gave written informed consent before participation. We performed all experiments according to relevant regulations. The Ethics Committee of the University Medical Center Göttingen approved the study (Application number: 36/4/19).

\subsubsection{Overview of the experimental sessions}

The design of the study is shown in Ошибка! Источник ссылки не найден.6. In the first session we collected neuroimaging data to determine the stimulation target and prepare anatomically realistic head models for electric field simulations. In the second session we estimated the motor cortical target and the resting motor threshold. In the remaining sessions, we performed three courses of rTMS-EEG (i.e., rhythmic, arrhythmic, and sham protocols) in a randomized order. In each rTMS-EEG session, we assessed the immediate effects and the aftereffects of rTMS with EEG and MEP. 


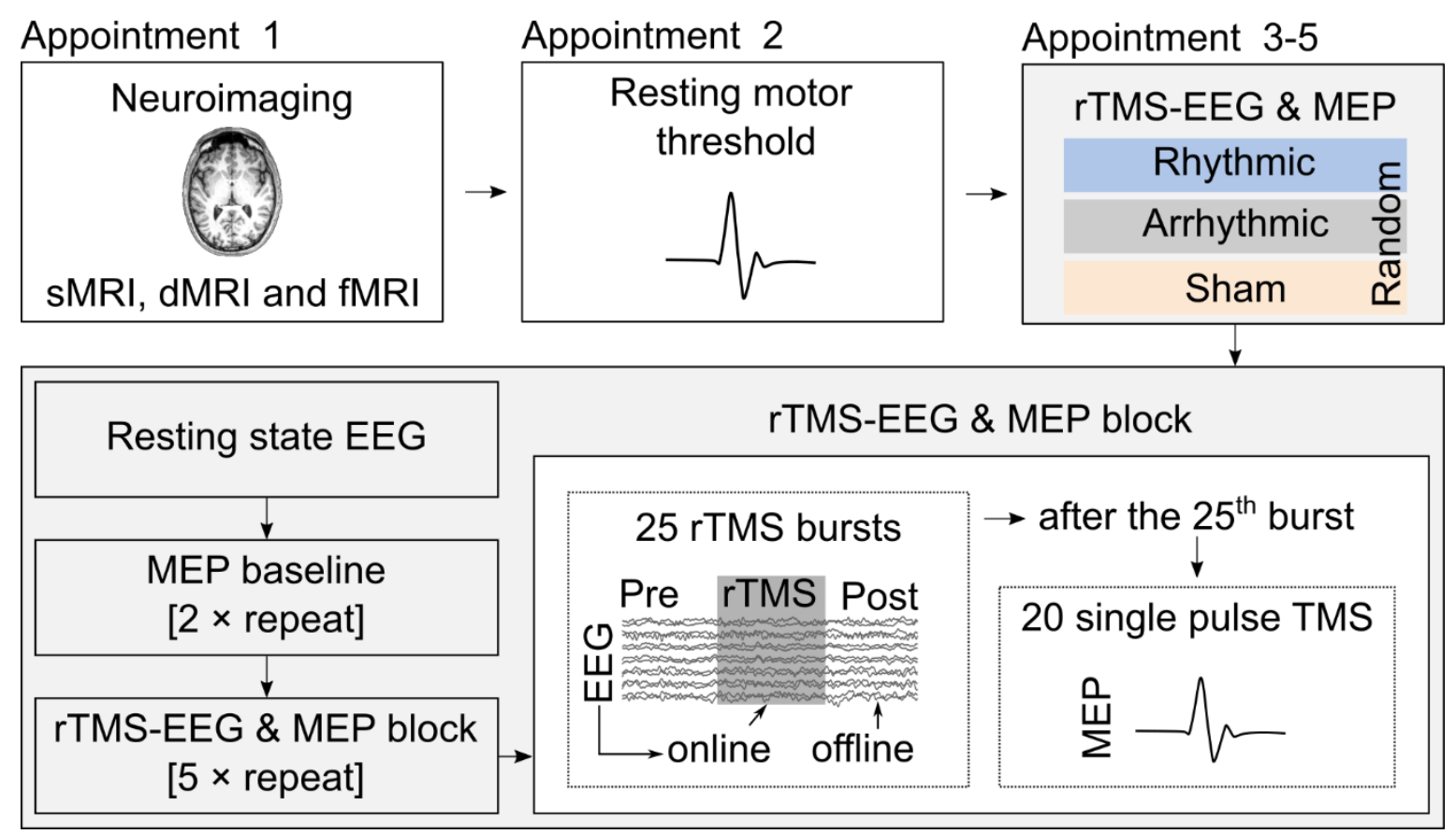

Figure 16. Schematic of study flow. After the neuroimaging and resting motor threshold estimation sessions, the participants took part in three rTMS sessions. Each session started with resting state EEG acquisition followed by determining the MEP baseline. Then, the participants received five rTMS-EEG and MEP blocks. In each block, we assessed the online and offline EEG effects. At the end of each block, we assessed cortical excitability by single pulse TMS.

\subsubsection{TMS and neuronavigation}

In both single-pulse TMS (spTMS) and rTMS the biphasic pulses were delivered using a MagPro X100 stimulator (MagVenture, Denmark) with a standard figure-eight coil (MC-B70), normal coil current direction, and $280 \mu$ s pulse duration. During stimulation, the participants sat in a comfortable chair with a chin and head fixator to minimize head movements. The TMS coil was placed over the motor cortex representation of the right first dorsal interosseous muscle, which we had previously identified as the highest local activation in the parametric t-map from the fMRI experiment. 
To monitor the coil position during stimulation we used an MRI-based real-time neuronavigation system (Brainsight TMS Navigation, Rogue Resolutions Ltd) and coupled it with a Polaris Vicra infrared camera (NDI, Waterloo, Canada).

\subsection{4. rTMS protocols}

We performed three rTMS-EEG sessions with the individually predefined parameters of stimulation for the rhythmic, arrhythmic, sham stimulation protocols. The order of the protocols was randomized for each participant. The sessions were performed on three different days with at least 72 hours between each.

The rTMS bursts were delivered at the prospectively estimated individualized intensities (see Head modeling and EF calculations). We set the intra-burst frequency of the rTMS bursts according to the individual alpha frequency (IAF) in the rhythmic and sham sessions. IAF was determined from the resting state continuous EEG data (see rTMS-EEG data acquisition). The intra-burst frequency used in the arrhythmic protocol was predefined by pseudorandomization of frequencies excluding $8-12 \mathrm{~Hz}$ and their harmonics ${ }^{72}$.

In the rhythmic and arrhythmic protocols the coil position and angle were adjusted to the optimal for inducing the MEP response. In the sham protocol the coil was positioned at the M1 but was tilted by 90 degrees away from the head surface. In each protocol, a burst consisted of 20 TMS pulses with an inter-burst interval of 10 or $11 \mathrm{~s}$. Each burst was repeated 25 times in a block. Each session consisted of five rTMS blocks, which resulted in 2,500 $(=20 \times 25 \times 5)$ pulses per session. For the stimulation parameters, see Ошибка! Источник ссылки не найден..

\begin{tabular}{l|lll}
\cline { 2 - 4 } & Rhythmic & Arrhythmic & Sham \\
\hline Coil handle orientation & Parallel & Parallel & Perpendicular \\
Stimulation intensity & Individualized & Individualized & Individualized \\
Range of MSO \% & 15 to 18 & 15 to 18 & 15 to 18 \\
Stimulation frequency & IAF & Pseudorandom & IAF \\
\cline { 2 - 4 } & & &
\end{tabular}




\begin{tabular}{l|lll}
\cline { 2 - 4 } IAF Hz $($ mean $\pm \mathrm{SD})$ & $10.3 \pm 0.9$ & $10.2 \pm 0.9$ & $10.5 \pm 0.6$ \\
Pulses / burst & 20 & 20 & 20 \\
Number of bursts/block & 25 & 25 & 25 \\
Number of blocks/session & 5 & 5 & 5 \\
Total pulse number & 2,500 & 2,500 & 2,500 \\
\hline
\end{tabular}

Table 6. Overview of rTMS stimulation conditions and stimulation parameters. Abbreviations: IAF - individual alpha frequency, MSO - maximum stimulator output.

\subsubsection{Head modeling and EF calculations}

We performed individual, anatomically realistic head modeling, and EF calculations using the Simulation of Non-invasive Brain Stimulation (SimNIBS) software package ${ }^{122}$. We created the multi-compartment head models using the 'mri2mesh()' SimNIBS function based on T1-, T2- weighted images with and without fat suppression (see MRI and fMRI data acquisition). We assigned the standard conductivity values to the compartments ${ }^{194}$, in S/m: scalp (0.465), bone (0.01), cerebrospinal fluid (1.654), gray matter (0.275) and white matter (0.126).

For each participant, we simulated the EF induced at the target determined from the fMRI activation map with a standard figure-eight coil (MC-B70). The coil was positioned at $8.5 \mathrm{~mm}$ from the head surface (including the $8 \mathrm{~mm}$ EEG electrode thickness) with a handle direction of ca. $45^{\circ}$ along the medium plane. We ran the calculations for intensities in the range of $15-20 \%$ of maximum stimulator output (MSO) and chose the intensity that induced around $35 \mathrm{mV} / \mathrm{mm}$ absolute peak EF (99.9\% percentile over the entire gray matter). The average intensity used for the rTMS protocols was $16.6 \pm 1.1 \%$ MSO.

The simulation of EF was performed twice: before and after rTMS-EEG sessions. The second estimations of EF were performed after stimulation using session specific coil position information exported from the Brainsight TMS Navigation software. The MNI coordinates of coil location and direction were transformed into subject-specific space using the ‘mni2subject_coords()' SimNIBS function. 


\subsubsection{MRI and fMRI data acquisition}

We acquired anatomical, diffusion-weighted, and functional magnetic resonance imaging data with a 3 T MRI-scanner (Siemens Magnetom TIM Trio, Siemens Healthcare, Erlangen, Germany). Functional MRI was collected during rhythmic, stereotypic movement using the first dorsal interosseous muscle, i.e. movement of forefinger from side to side. The fMRI data preprocessing was performed with the Statistical Parametric Mapping (SPM 12, Welcome Department of Imaging Neuroscience, London, UK) software package implemented in MATLAB software. Following preprocessing described elsewhere ${ }^{72}$, the general linear model was applied at the single-subject level. Voxels were identified as significant if $p<0.05$ (familywise error corrected for multiple comparisons on the voxel level). The individual activation Tmap as well as T1 image were uploaded to the neuronavigation system.

\subsubsection{EMG acquisition}

We recorded the EMG with an $\mathrm{Ag}-\mathrm{AgCl}$ electrode pair attached to the first dorsal interosseous muscle of the right hand in a belly-tendon montage. Signals were sampled at $5 \mathrm{kHz}$, amplified and bandpass filtered between $2 \mathrm{~Hz}$ and $4 \mathrm{kHz}$, and digitized using a $1401 \mathrm{AD}$ converter (CED 1401, Cambridge, UK). All EMG measures were recorded with Signal software (CED, version 4.08).

The experiment started by determining the resting motor threshold (RMT) at the hotspot. The initial position was defined as that with the fMRI local activation maximum derived from the parametric t-map at the anatomical hand knob formation. The spTMS was delivered at 0.25 $\mathrm{Hz}$ by placing the stimulation coil orthogonally to the central sulcus at $30 \% \mathrm{MSO}$. We increased the intensity in increments of 2\% MSO until the stimulation evoked MEPs. We further decreased or increased intensity by $1 \%$ MSO until we identified the lowest intensity that evoked at least 
five out of ten MEPs with a peak-to-peak amplitude $>50 \mu \mathrm{V}$. The average RMT was $44.0 \pm 8.5 \%$ of MSO.

In the rTMS-EEG sessions we determined the $1 \mathrm{mV}$ MEP threshold that we used for the baseline MEP measurements and for assessing the corticospinal excitability level after rTMS. To find the $1 \mathrm{mV}$ threshold, we delivered 20 TMS pulses initially at $120 \%$ of RMT. We then averaged the peak-to-peak MEP amplitudes and evaluated whether the $1 \mathrm{mV}$ threshold was reached. In the next block, we decreased or increased the TMS intensity by 5\% RMT, if necessary. We repeated this procedure until we obtained a peak-to-peak MEP amplitude within the range of $0.8-1.2 \mu \mathrm{V}$.

We performed the baseline measurements twice to ensure that the baseline MEP amplitudes were accurately assessed. In each measurement, we delivered 20 TMS pulses. Following the baseline measurement and immediately after each rTMS block, we assessed the MEPs using the $1 \mathrm{mV}$ threshold intensity.

\subsection{8. rTMS-EEG data acquisition}

EEG acquisition. In each rTMS-EEG session we recorded the EEG simultaneously with rTMS from $64 \mathrm{Ag} / \mathrm{AgCl}$ active EEG electrodes (actiCAP slim, BrainVision LLC, Germany) at a $2.5 \mathrm{kHz}$ sampling rate without hardware filters (actiChamp, Brain Vision LLC, Germany). Ground and reference electrodes were located at $\mathrm{AFz}$ and $\mathrm{FCz}$, respectively. Impedance values were maintained below $20 \mathrm{k} \Omega$.

Stimulation sessions started with recording two blocks of continuous resting state EEG for four minutes with eyes open and eyes closed. During the recording we instructed the participants to sit calmly and relaxed, minimize blinking and horizontal eye movements, not to move their arms, legs, or facial muscles, to avoid any calculations or repetitive mental activity such as reproducing any texts, lyrics, or melodies. The participants wore QuietControl 30 wireless 
headphones with active noise reduction and white noise masking during all recordings (Bose Corporation, USA). The volume level was always kept below the manufacturer's recommended safety limits. This procedure minimized but did not eliminate the sound produced by the TMS stimulus. Therefore, at the end of each session participants were asked to evaluate the click sound by scale from -100 to +100 percent, where zero meant the same loudness of click and white noise. The coordinates of each electrode were saved to the neuronavigation system once at the end of the final rTMS-EEG session.

We performed offline data analysis with the FieldTrip toolbox for EEG- and MEG analysis [version 20170119; 85; http://fieldtrip.fcdonders.nl] as described in reference ${ }^{72}$. We determined the global peak alpha frequency in the range of $8-12 \mathrm{~Hz}$ in the eyes open state. For three participants the peak frequency during the eyes open state was not clearly defined. We, therefore, chose the stimulation frequency from the global peak alpha defined from the eyes-closed state.

The EEG data of each participant consisted of resting-state EEG at the beginning and the end of each session; 140 trials of spTMS (40 trials from baseline measurements and 20 trials after each rTMS block) and 125 trials of rTMS-EEG recording.

\subsubsection{Data analysis}

EF analysis. Descriptive statistics of the absolute EFs and its normal component were performed in MATLAB for three predefined anatomical regions of interests (ROIs), i.e., the left precentral and left postcentral gyri, and left central sulcus. From each ROI, we extracted the robust minimum $(0.1 \%)$ and maximum $(99.9 \%)$ values, as well as the global mean.

MEP analysis. EMG recordings were converted with cfs2mat utility (https://github.com/giantsquidaxon/cfs2mat) to MATLAB '.mat' format where they were further preprocessed. The data were visually inspected for activation higher than $50 \mu \mathrm{V}$ in the $80 \mathrm{~ms}$ 
pre-TMS interval. Trials containing pre-activation were excluded from further analysis. Altogether $2.1 \%$ of all MEP trials were excluded from the dataset.

Peak-to-peak MEP amplitude was calculated for 10-50 ms after the TMS pulse. We transformed the MEP data using Matlab's base 10 logarithm function ' $\log ()$ ' to reduce the frequency and the weight of outliers, and to improve variance homogeneity across experimental conditions. After log transformation we removed four additional data points because they exceeded $\pm 3 \mathrm{SD}$ of the global mean value.

The MEP amplitudes were averaged across the blocks at the time points. The effect of rTMS protocols on cortical excitability was tested using the linear mixed-effect model implemented in $\mathrm{R}$ (version 4.3.0), R-Studio integrated development environment (version 1.3.1093), and 'lmerTest' package (version 3.1.2) ${ }^{195-197 .}$

The base model only included the random intercept for the participant using the formula described in Table 7. In the next model we added the effect of Protocol (three levels: rhythmic, arrhythmic, and sham rTMS) and Time (six levels: baseline and five subsequent measurements).

\begin{tabular}{ll}
\hline Model & Formula \\
\hline Base & $\log 10(\mathrm{MEP}) \sim(1 \mid$ participant $)$ \\
1 & $\log 10(\mathrm{MEP}) \sim(1 \mid$ participant $)+$ protocol \\
2 & $\log 10(\mathrm{MEP}) \sim(1 \mid$ participant $)+$ protocol + time
\end{tabular}

Table 7. The formulae of the tested models.

We used the 'anova()' function to test which model provided the best parsimonious data fit. Models with a p-value of 0.05 or less were considered to be significantly better than the previous model. On the winning model, we ran the 'anova()' function to perform Type III Analysis of Variance with Satterthwaite's method. 


\subsubsection{0. $\quad$ rTMS-EEG analysis}

EEG preprocessing. The EEG data were preprocessed offline in MATLAB (2017b, Mathworks) using Fieldtrip toolbox (v.20180114, ${ }^{126}$ ) and custom-written code. The data were cleared of TMS-induced artifacts by the same procedure as described in ${ }^{72}$. The data were then re-referenced to a common reference and were cut into segments of $8.5 \mathrm{sec}$ length. Trials were aligned to the last TMS pulse of the burst and contained $5.5 \mathrm{sec}$ before and $3 \mathrm{sec}$ after the pulse.

The data were visually inspected to identify and remove excessively noisy channels and trials with jumps or muscle artifacts. The data were resampled to $1250 \mathrm{~Hz}$, and the signals relating to eye blinks and eye movements were identified and removed by the second ICA. On average, $15.8 \pm 4(\mathrm{R}) ; 16.7 \pm 3.6(\mathrm{AR}) ; 15.8 \pm 4.2(\mathrm{SH})$ components and $6.2 \pm 2(\mathrm{R}) ; 4.2 \pm 1.3(\mathrm{AR}) ; 3.8$ $\pm 1.5(\mathrm{SH})$ channels were removed from the data. The remaining clean dataset contained on average $103 \pm 6.5(\mathrm{R}) ; 107.8 \pm 9(\mathrm{AR}) ; 101.7 \pm 13.5(\mathrm{SH})$ trials.

Phase-locking value (PLV). We calculated the degree of synchronization between the ongoing signal phase and the TMS pulses by estimating the PLV during the rTMS burst ${ }^{91}$. We used our previous analytical pipeline described elsewhere ${ }^{72}$. Briefly, we simulated the sinusoidal wave at the stimulation frequency and aligned its phase to TMS pulses. The simulated signal was appended with cleaned EEG data as an additional channel. The data was transformed by complex Morlet wavelet decomposition from 1 to $25 \mathrm{~Hz}$.

PLV was computed between the phases of the original signal and the simulated wave. The PLVs were normalized relative to the baseline at $500 \mathrm{~ms}$ before TMS bursts onset. Then we focused the analysis on the online effect which showed the changes in PLVs during stimulation and the offline effect which showed PLV changes immediately after TMS bursts. We averaged normalized PLVs separately for online and offline effects over time from -2 to $0 \mathrm{sec}$ and from 0 to $2 \mathrm{sec}$ correspondingly. They were further compared between protocols by group-level, nonparametric, cluster-based permutation test $(10,000$ permutations, two-tailed, significance 
accepted at $\mathrm{p}<0.05$ ). We performed two dependent $\mathrm{t}$-tests to compare rhythmic with arrhythmic and sham separately for online and offline effects.

Power analysis. Time-frequency transformed data were further investigated for power changes. Residual TMS artifacts could affect the signal amplitude and power ${ }^{72}$, and we, therefore, performed power analysis on artifact-free intervals from 0.2 to 2 sec after an rTMS burst. The power was extracted as a real part of complex Wavelet decomposition and averaged over all channels for the three protocols. The trials were split for 'before' and 'after' intervals that corresponded to $1.8 \mathrm{sec}$ prior and post rTMS. The difference in power was statistically tested using activation versus baseline t-statistic ('actvsblT' in Fieldtrip). The analysis was performed on all sensors for $5-25 \mathrm{~Hz}$ frequency. Statistical significance was assessed by nonparametric, cluster-based permutation test (10,000 permutations, two-tailed, significance accepted at $\mathrm{p}<$ $0.05)$.

Next, we investigated the single-trial alpha power by reconstructing the virtual source signal at the cortical level. For that we created a subject-specific virtual channel using the linear constrained minimum variance (LCMV) beamformer approach ${ }^{198}$. The coordinates of the source corresponded to the 'hotspot' of the stimulation site, which was exported from EF estimations (see section 5.3.6) in subject-specific space.

A realistic three-layer volume conduction model was constructed using the individual MRI using the boundary element method ${ }^{199}$. A grid with $10 \mathrm{~mm}^{2}$ resolution was created per individual, which was subsequently normalized to MNI space. The spatial filter was constructed from the full trial length and then was used to reconstruct the virtual source signal. Timefrequency decomposition was estimated for 1-30 Hz using complex Morlet wavelet. The wavelets contained seven cycles with a three-Gaussian window. The decomposition was performed for the trial interval from 3.5 before to $2.5 \mathrm{sec}$ after the TMS offset. The virtual source power was compared between active protocols (rhythmic, arrhythmic) versus sham by cluster- 
based permutation statistical test $(10,000$ permutations, two-tailed, significance accepted at $\mathrm{p}<0.05)$.

\subsection{Results}

In the present study, we assessed the effects on the EEG during rTMS bursts and the aftereffects in the inter-burst intervals, i.e., immediately after the rTMS bursts. We applied phase and power-based analysis and reconstructed the signal of the virtual source placed in the grey matter of the stimulated target. Moreover, we tested the aftereffects of rTMS on the corticospinal excitability level by analyzing the size of the MEP amplitudes after the end of the rTMS block, i.e., after 25 rTMS bursts.

\subsubsection{Increased mu-alpha synchronization during and after rhythmic rTMS}

First, we characterized the amount of synchronization during the rTMS bursts (online) and immediately after the stimulation cessation (offline; see Ошибка! Источник ссылки не найден.). We estimated PLVs that represent the amount of consistency of the EEG signal phaselocked to the external TMS pulses ${ }^{200}$.

During the rTMS bursts (online), a nonparametric cluster-based permutation statistical test revealed a significant increase in PLVs in the $\mu$-alpha range 8-13 Hz comparing the rhythmic versus arrhythmic $(\mathrm{p}<0.001)$ and rhythmic versus sham $(\mathrm{p}<0.001)$ rTMS protocols (see Figure 17A). In the rhythmic versus arrhythmic comparison, we found the largest difference in PLV increase in the posterior $\left(\mathrm{T}_{\text {peak }}(14)=4.27\right)$ and the left frontal $\left(\mathrm{T}_{\text {peak }}(14)=4.46\right)$ regions $($ see Figure 17B, left). When rhythmic rTMS was compared to sham rTMS protocol, we found the highest PLV increase in the left central region $\left(\mathrm{T}_{\text {peak }}(13)=4.03\right.$; see $17 \mathrm{~B}$, left). Rhythmic rTMS 
synchronized ongoing sensorimotor $\mu$-alpha rhythms as indicated by increased phase-locking values.

A
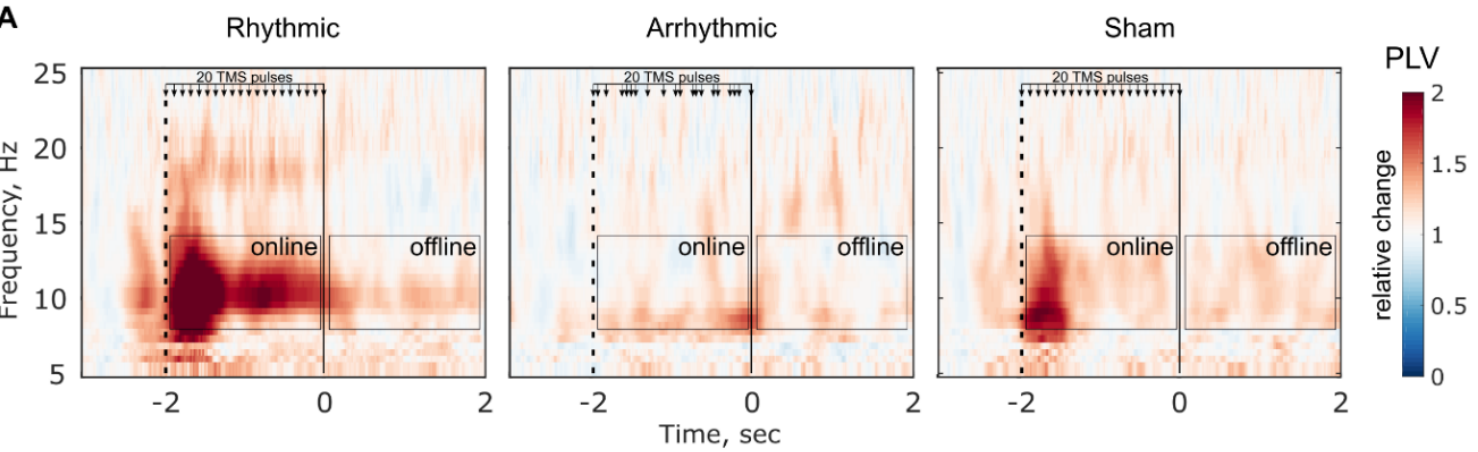

B

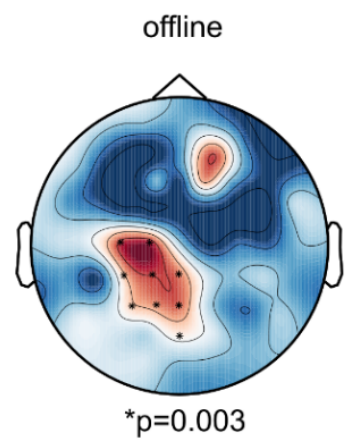

C
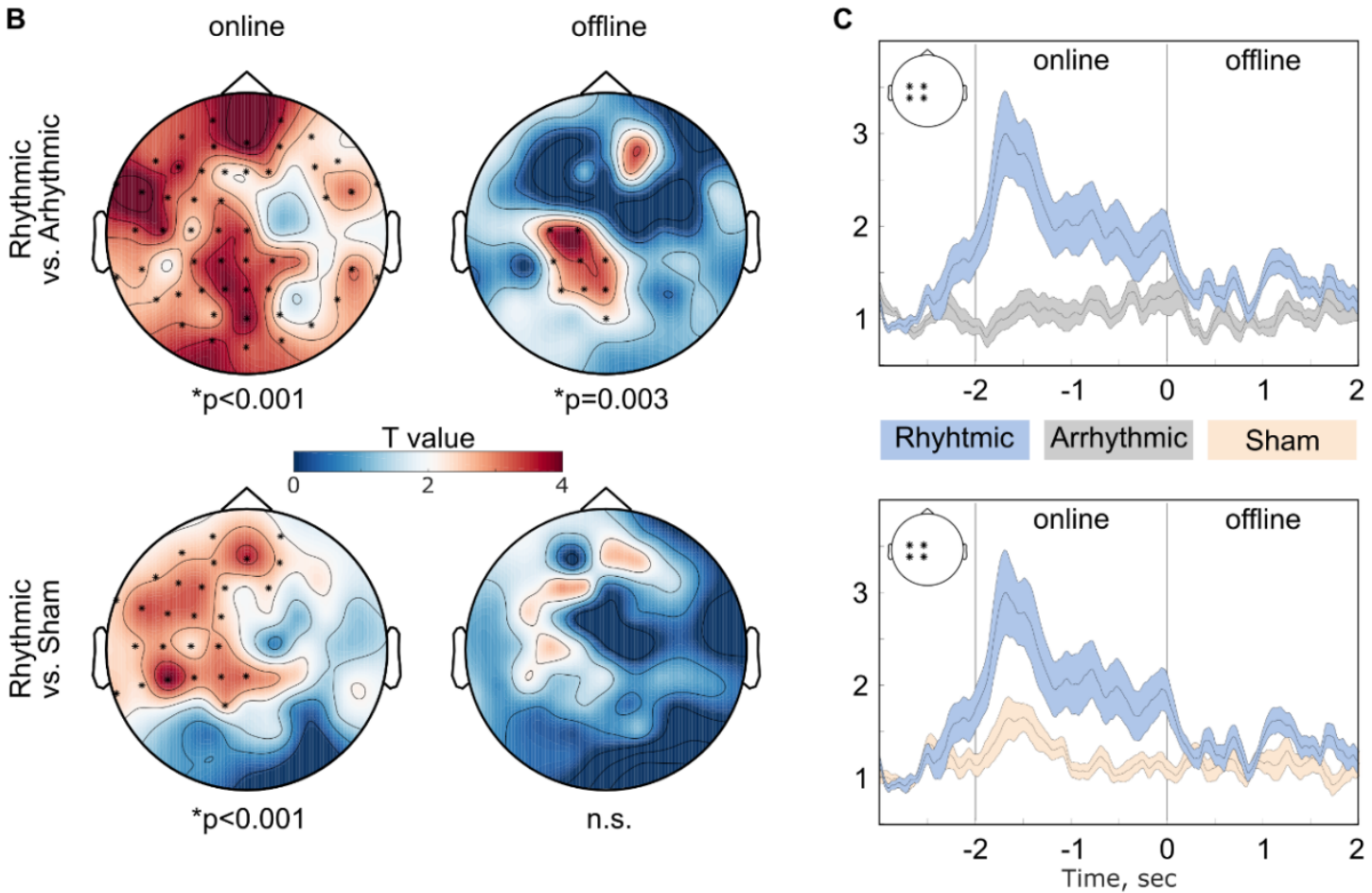

Figure 17. Rhythmic rTMS synchronized ongoing $\mu$-alpha rhythms indicated by increased phaselocking values near the stimulation target. A) Global time-frequency representation of PLVS during and after rTMS burst for rhythmic, arrhythmic, and sham protocols (from left to right). PLVs are normalized by $500 \mathrm{~ms}$ interval before rTMS onset. B) Statistical tests revealed a significant increase of $\mu$-alpha synchronization during rhythmic rTMS compared with arrhythmic or sham protocols. Immediately after rhythmic rTMS burst, $\mu$-alpha synchronization is increased compared wiht arrhythmic but not with sham protocol. C) Time course of PLVS averaged over four left central electrodes (C1, C3, CP1, CP3). 
Immediately after rTMS bursts (offline) we found a significant difference only between the rhythmic and arrhythmic protocols $(\mathrm{p}=0.003)$ with a peak $\mathrm{t}$-value over the central electrodes $\left(\mathrm{T}_{\text {peak }}(13)=3.71\right)$ on the stimulation site (see Ошибка! Источник ссылки не найден.17В, right and Ошибка! Источник ссылки не найден.17C). The difference between rhythmic versus sham was not significant.

\subsubsection{Rhythmic rTMS increased alpha/beta power after stimulation}

We then investigated $\mu$-alpha power changes induced by rTMS protocols. We estimated the relative change of global power by time-frequency transformation. The $\mu$-alpha power was statistically compared between 'after' and 'before' EEG intervals. These intervals were free of any residual TMS artifacts. Statistical tests revealed significant clusters for rhythmic $\left(\mathrm{T}_{\text {peak }}(14)\right.$ $=1.64, \mathrm{p}=0.01)$, arrhythmic $\left(\mathrm{T}_{\text {peak }}(13)=1.87, \mathrm{p}=0.002\right)$ condition but not for the sham $\left(\mathrm{T}_{\text {peak }}\right.$ $(14)=0.99, p=0.24)$ protocol (see Ошибка! Источник ссылки не найден.18).
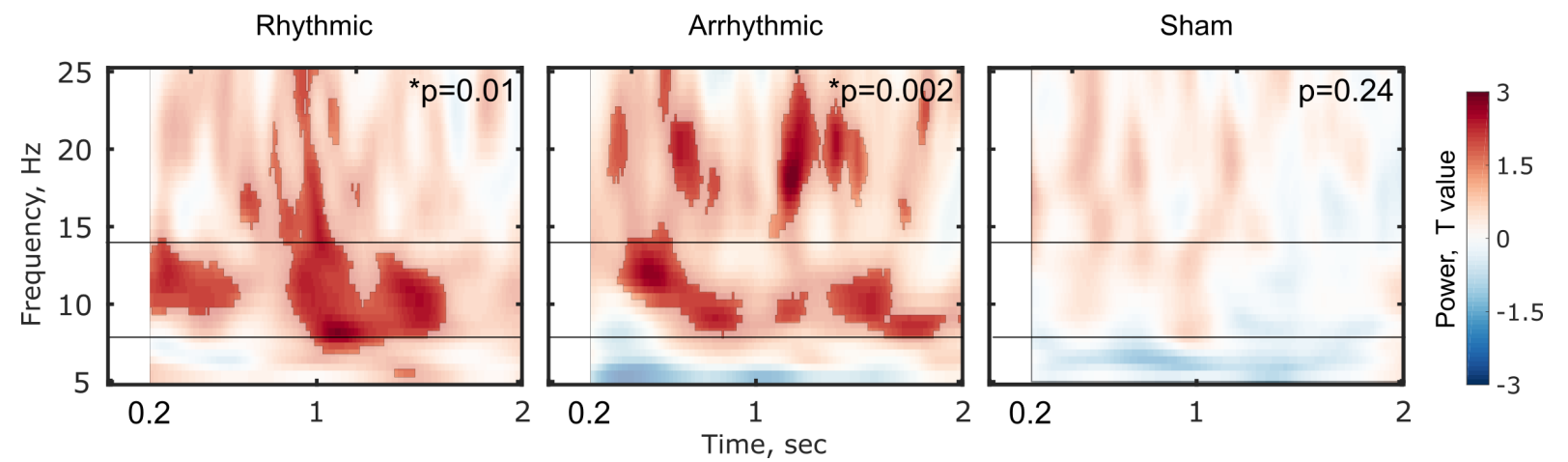

Figure 18. Active stimulation protocols (rhythmic and arrhythmic) increased power in $\mu$-alpha and beta frequency ranges immediately after stimulation (inter-burst intervals). Sham rTMS applied at IAF did not change the power of $\mu$-alpha rhythm. Time-frequency plots are masked with $p<0.05$. 
To investigate the location-specific power change we projected the sensor level EEG to the source space by reconstructing the virtual channel signal (see Ошибка! Источник ссылки не найден.19). The source location was selected on the cortex surface with coordinates corresponded to the peak EF, which were estimated individually (see Ошибка! Источник ссылки не найден.19B). The statistical test of time-frequency transformation of the virtual channel revealed a significant increase in the $\mu$-alpha $(\mathrm{T}(14)=0.93, \mathrm{p}=0.040)$ and beta $(\mathrm{T}(14)$ $=1.05, \mathrm{p}=0.032$ ) power (see Ошибка! Источник ссылки не найден.19C). Although the arrhythmic protocol increased the global and virtual source power, it was not significantly different from the sham protocol $(\mathrm{T}(13)=0.75, \mathrm{p}=0.065$; see Ошибка! Источник ссылки не найден.19С).

A

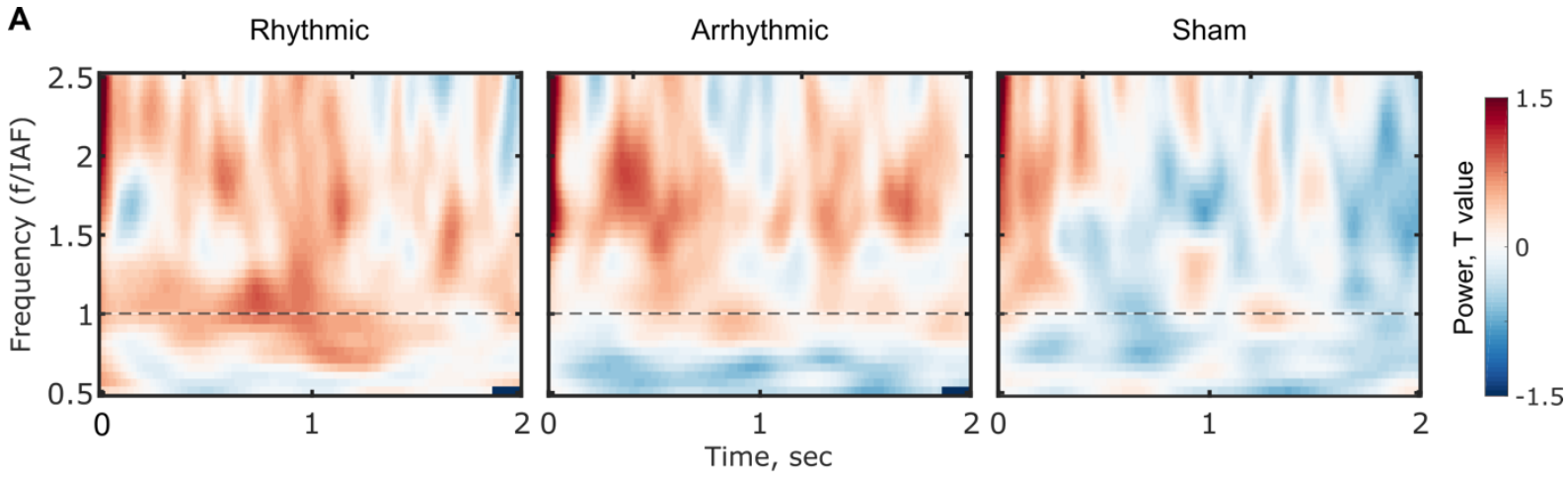

B

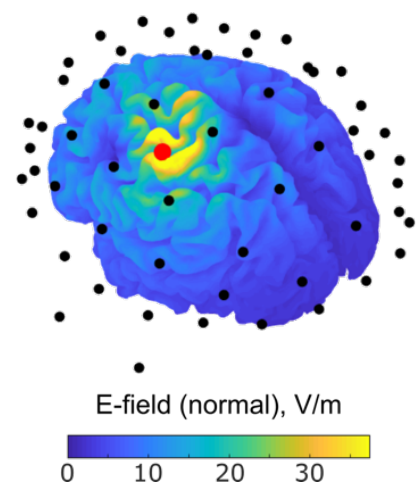

C

Rhythmic versus sham

Arrhythmic versus sham

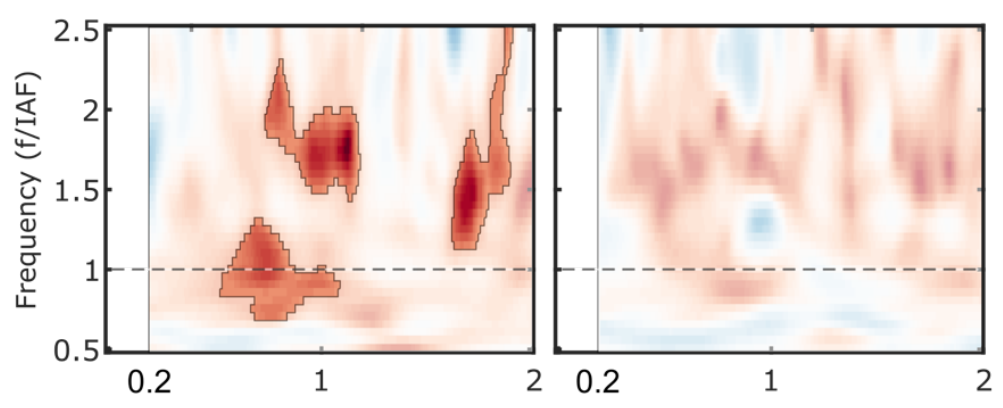

Figure 19. Rhythmic rTMS protocol increased local $\mu$-alpha and beta power at the stimulation target. A) Time-frequency representation of relative power. The frequency scale is normalized to stimulation frequency (at IAF). Value one on the ordinate corresponds to IAF. B) Example of source localization for a single participant. The head model and EF estimations were used for 
locating the virtual source in the peak EF induced by TMS shown as a red sphere. Black dots show the location of the EEG electrodes. C) Statistical maps of the time-frequency representation for the virtual source. Significant clusters $(p<0.05)$ are marked by contour lines.

\subsubsection{Rhythmic rTMS decreased corticospinal excitability level}

We assessed the changes in cortical excitability by focusing on the log-transformed peakto-peak MEP amplitudes. The winning model included the random intercept for participants and the fixed effect for the Protocol $\left(\chi^{2}(1, N=3)=9.9466, \mathrm{p}=0.0069\right)$, the latter of which had a significant main effect $(F(2,244.33)=5.0113, p=0.0072)$. Further analysis revealed that relative to sham stimulation, rhythmic rTMS significantly reduced the MEP amplitudes $(t=-3.171, d f=243.27, p=0.0017)$. On the other hand, arrhythmic rTMS had no significant effect on the MEP amplitudes $(t=-1.467, d f=244.85, p=0.1435)$. The Bonferroni-adjusted alpha levels were 0.0034 and 0.2870 for the rhythmic and arrhythmic protocols, respectively.

Then, we focused on the rhythmic protocol and studied the correlations between the MEP amplitudes and the degree of $\mu$-alpha synchronization and the spectral power changes (see Ошибка! Источник ссылки не найден.20). None of the correlations were significant (all pvalues $>0.355)$. 

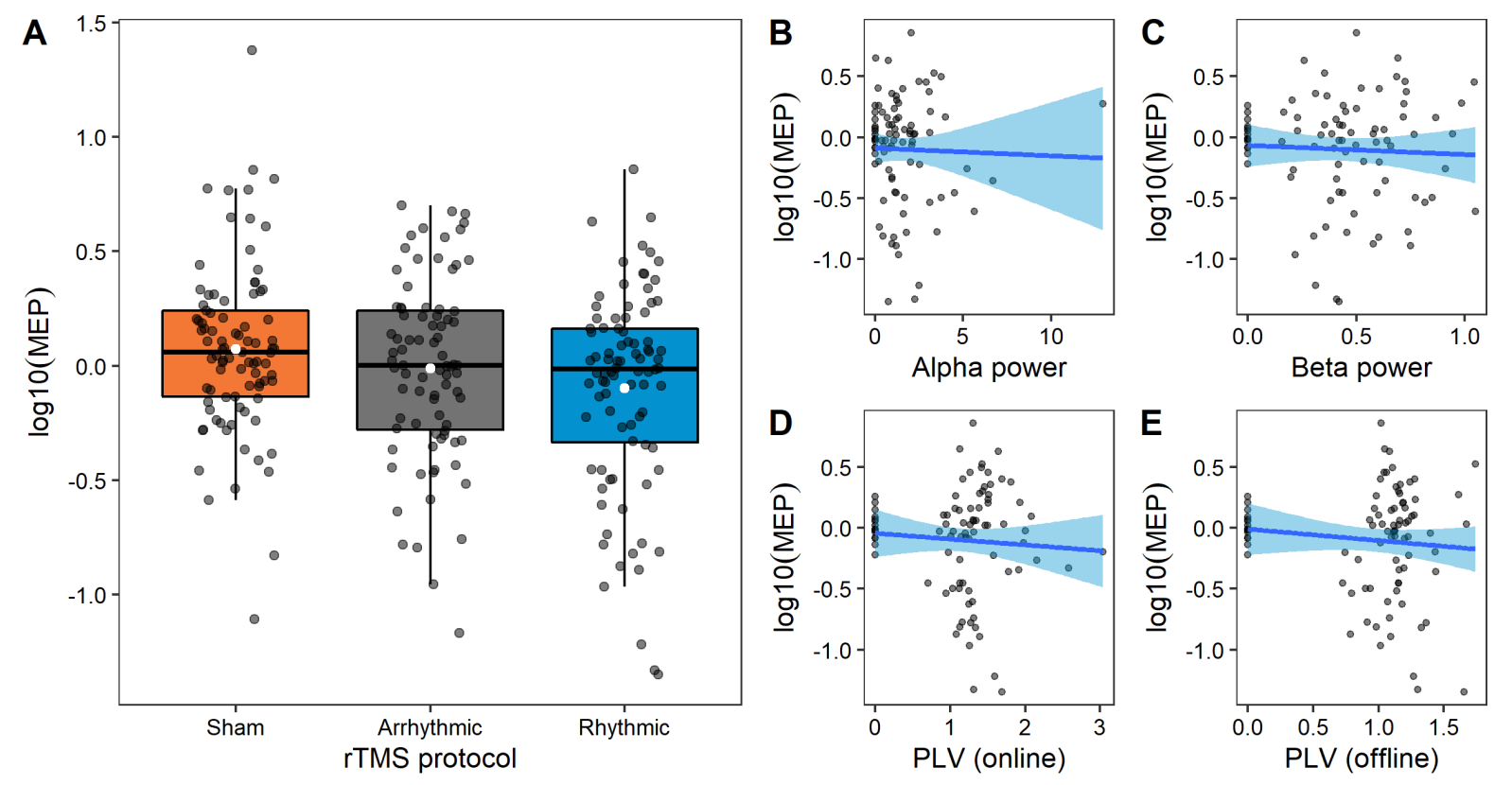

Figure 20. Rhythmic rTMS decreased the MEP amplitude. A) Log-transformed MEP amplitudes according to the rTMS protocols. White dot shows the mean values, black dots represent individual measurements. B-E) Linear relationships between the individual log-transformed MEPS and the individual EEG parameters. Alpha and beta power values (top) were extracted from virtual source time-frequency analysis as a peak power (normalized to baseline) for $\mu$ alpha $(I A F \pm 1 H z)$ and beta rhythm $(15-20 \mathrm{~Hz})$ for 0.2-2 sec after rhythmic rTMS bursts. PLV values (bottom) are averaged over four channels (C1, C3, CP1, CP3) at the stimulation location at the $I A F \pm 1 H z$.

\subsection{Discussion}

In the present study, we demonstrated that low-intensity rTMS over the left M1 influenced both oscillatory activity and corticospinal excitability level that outlasted the stimulation period. Furthermore, we replicated our previous findings on entrainment of parietal-occipital alpha rhythm by weak rTMS-induced EFs [10], now at the motor cortex, and we extended our previous work by showing that rhythmic rTMS enhanced sensory-motor $\mu$-alpha rhythm and decreased corticospinal excitability. 


\subsubsection{Electrophysiological effects of low-intensity rTMS}

One question of key importance in applying rTMS is the selection of the stimulation intensity [15]. In the present study we used the prospective EF estimation approach where we defined the stimulation intensity using computational models of the rTMS induced EF. We showed that low-intensity rTMS at an individual $\mu$-alpha frequency over the left occipital cortex [10] and now over the left motor cortex increased ongoing neural synchrony.

We extended the EEG analysis to the induced effects on the phase and power of the $\mu$ alpha rhythm after the rTMS bursts. We found that the rTMS- increased neural synchrony in M1 lasted for up to two seconds after applying rhythmic but not arrhythmic or sham rTMS. The explored intervals were free from the TMS-produced artifacts, such as decay, ringing, or muscle artifacts.

Moreover, the degree of $\mu$-alpha synchrony was significantly higher after rhythmic than after arrhythmic rTMS, though they were not significantly different compared with sham rhythmic rTMS. This finding most likely indicates that the sound from rhythmic TMS clicks contributed to the increase in $\mu$-alpha synchronization ${ }^{70}$. However, the increase after sham was much lower than after real rTMS in both online and offline intervals. Therefore, we believe that the observed effect did not originate solely from the rhythmic rTMS clicks sound. Moreover, we did not find $\mu$-alpha synchronization in the temporal electrodes, which would indicate the entrainment of $\mu$-alpha rhythm through auditory input. Based on these grounds, we conclude that rhythmic rTMS can induce and maintain the synchronized oscillatory activity at the stimulation target.

Furthermore, we investigated global and local power changes for up to two seconds after the rTMS bursts. We observed increased global alpha power following rhythmic and arrhythmic but not sham rTMS. Here the maintained oscillatory activity resulted not only in the phase- 
locking changes but also in the increased power of $\mu$-alpha $(8-14 \mathrm{~Hz})$ and beta $(15-20 \mathrm{~Hz})$ frequency ranges.

This was not the case for local alpha power at the source level where we found the alpha and beta power increase only in rhythmic rTMS. Our findings are in line with previous studies where participants received conventional stimulation intensities of rTMS (80-100\% of resting motor threshold) applied over the left M1 at rest ${ }^{201,202}$. The authors found a significant difference between the real and sham rTMS in mu (10-12 Hz) and beta $(13-30 \mathrm{~Hz})$ power for $5 \mathrm{sec}$ after real rTMS trains.

\subsubsection{Arrhythmic rTMS induces alpha perturbation in the occipital but not the motor cortex.}

One interesting finding is that arrhythmic rTMS induced different aftereffects in the occipital and sensorimotor $\mu$-alpha rhythms ${ }^{73}$. Whereas arrhythmic rTMS significantly suppressed parietal-occipital alpha ${ }^{73}$, in the present study it increased the sensorymotor $\mu$-alpha power (see Figure 3). Apart from the stimulation target, we used closely matched stimulation parameters in the two experiments. On the other hand, rhythmic rTMS increased the alpha power in both experiments.

The reason for this finding is currently not well understood. One crucial difference between the studies was that the amplitude of alpha rhythm was stronger and the peak alpha frequency was more prominent at the occipital than at the sensorimotor area. We speculate that the properties of the endogenous oscillation could have shaped the direction of the electrophysiological response to arrhythmic rTMS. In cortical regions with pronounced peak alpha frequency, arrhythmic perturbation suppressed alpha power. On the contrary, in cortical regions with less pronounced peak alpha frequency, arrhythmic rTMS increased alpha power. 
Nevertheless, further studies are needed to better understand the neural mechanisms of arrhythmic rTMS on ongoing oscillatory activity in different cortical regions.

\subsubsection{Low-intensity rTMS affects corticospinal} excitability

We also demonstrated that rhythmic rTMS inhibited the corticospinal excitability level as indicated by the reduced peak-to-peak MEP amplitudes. Contrary to our hypothesis, arrhythmic rTMS did not result in an MEP amplitude increase; we found no significant difference from the sham condition.

The observation that only rhythmic but not arrhythmic rTMS induced aftereffects in corticospinal excitability argues for the role of spike-timing-dependent plasticity. This form of Hebbian plasticity emerges by synchronously activating pre- and postsynaptic neurons. Because arrhythmic rTMS cannot achieve this tight temporal correlation between pre- and postsynaptic neurons, it did not induce lasting changes in corticospinal excitability.

In addition, we explored the relationships between MEP amplitudes and the EEG parameters such as the phase-locking value during and after the rTMS bursts as well as peak $\mu$ alpha and beta power at the stimulation target. However, we found that changes in MEP amplitudes after the rTMS block did not correlate with changes in the EEG during or shortly after the rTMS bursts.

Several rTMS studies have demonstrated increased MEP amplitudes after applying $10 \mathrm{~Hz}$ rTMS over the primary motor cortex ${ }^{190,203-205}$, whereas others reported decreased MEP amplitudes or no aftereffect ${ }^{190,193,201,204-206}$. Moreover, MEP amplitudes and EEG readouts

correlated only weakly (CorrCoef $<0.1)^{207,208}$. Therefore, it is likely that these outcome measures do not reflect the same neural mechanisms. 


\subsection{Conclusions}

In summary, we found that rTMS applied at an intensity of $\sim 40 \%$ RMT is effective to induce changes in ongoing electrophysiological and aftereffects in corticospinal excitability. The stimulation intensity used in the current study and in our previous studies $(38.8 \pm 6.5 \%$ RMT $)$ was approximately half of that applied in conventional rTMS studies, i.e., $80-120 \%$ of the motor

threshold ${ }^{71}$. However, more research on this topic needs to be undertaken before the association between electrophysiology and cortical excitability is more clearly understood. 


\section{General discussion}

Repetitive TMS, additionally to its therapeutic application, offers a possibility to interact with ongoing rhythms in an intact human brain. The standard method of determining the stimulation intensity is based on the evoked liminal response in the visual or motor system (e.g., resting motor or phosphene threshold). The vague magnitude of the resulting EF is the critical limitation of the standard approach. Therefore, we need a characterization of the EF strength induced by rTMS to improve the understanding of the neural mechanisms of rTMS. In this thesis, I attempted to better characterize EF's role in the neural entrainment in humans. I used individualized computational modeling of the prospective induced EF to define stimulation intensity. The key question of the thesis was whether rTMS applied at weak intensities between 2 to 4 times lower than usual $(25-50 \mathrm{mV} / \mathrm{mm})$ produces an entrainment effect. For the first time, rTMS was applied at such low intensities because previously, it was thought that TMS is not effective whenever it does not elicit an evoked potential. In three series of experiments, we demonstrated the consistent online entrainment effects and later power modulation of $\mu$-alpha rhythm and inhibition of the targeted motor cortex.

First, we applied rTMS over the left occipito-parietal area targeting alpha rhythm. Using an individual alpha frequency of rTMS and three intensities inducing peak EF of 20, 35, and 50 $\mathrm{mV} / \mathrm{mm}$ in the visual cortex, we demonstrated online synchronization of alpha activity expressed as phase alignment to the external pulses after rhythmic but not arrhythmic or sham protocols. We assumed that the induced changes could appear due to increased cortical inhibition via increased neural synchrony because low EF preferentially stimulates inhibitory circuits ${ }^{209}$. Further, we investigated whether the synchronized alpha was maintained after the end of stimulation bursts by estimating the changes in alpha power. According to the entrainment echo hypothesis, we expected increased alpha power after rhythmic TMS $^{63}$. Contrary to the 
hypothesis, we found a nil effect after rhythmic rTMS at all intensities and a significant reduction of alpha power post stimulation induced by arrhythmic rTMS at $35 \mathrm{mV} / \mathrm{mm}$.

\section{Parietal alpha rhythm, 8-13 Hz}

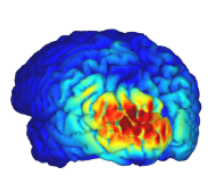

High amplitude of ongoing oscillations

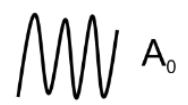

\section{Somatosensory $\mu$-alpha rhythm, 8-13 Hz}

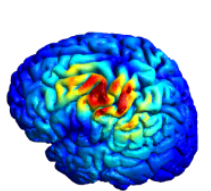

Low amplitude of ongoing oscillations

$\mathrm{WA}_{0}$
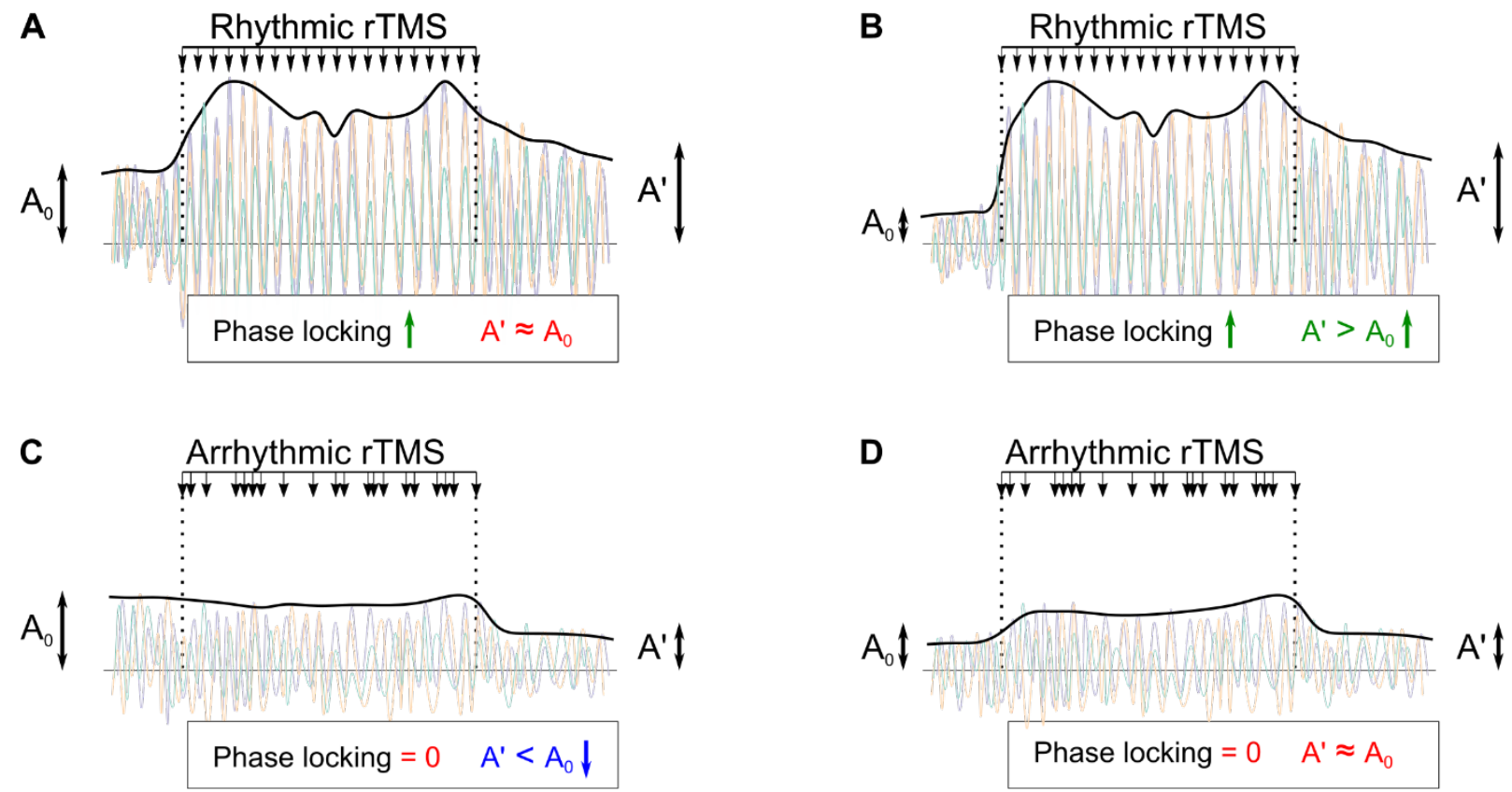

$\mathbf{E}$
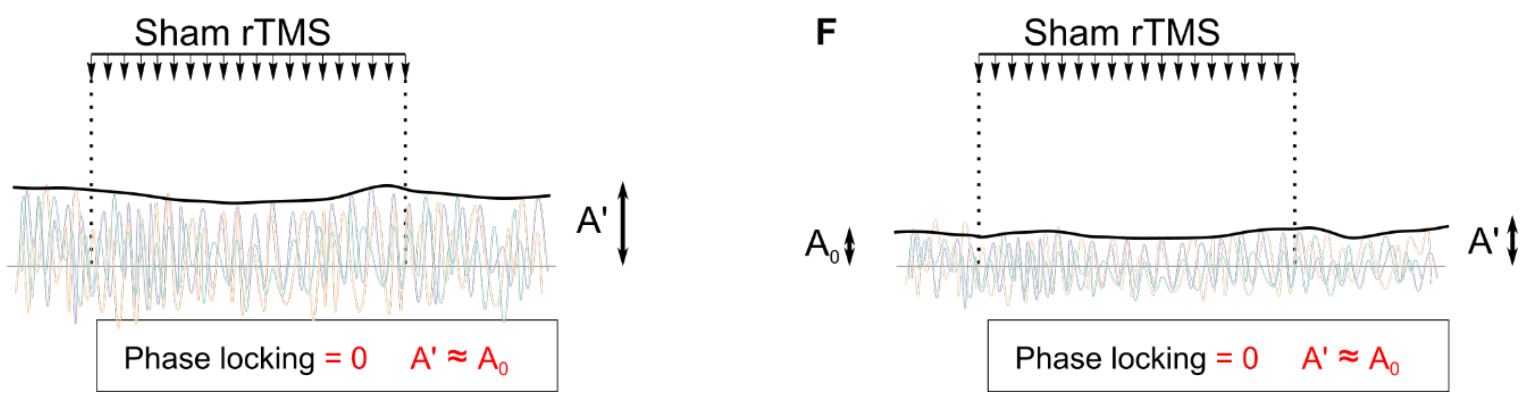

Figure 21. Illustration of entrainment effect induced by stimulation by $35 \mathrm{mV} / \mathrm{mm}$ rTMS over parieto-occipital (left column, Chapter 2-3) and motor (right column, Chapter 4) areas. Twenty pulses of rTMS applied in rhythmic (A-B), arrhythmic (C-D), and rhythmic sham (E-F) protocols. Signals are shown as an illustration of filtered EEG in the range of 8-13 Hz. The amplitude of baseline intact $\mu$-alpha rhythm in the parietal region higher than in the motor cortex; therefore, the induced power modulation might be different, for example, arrhythmic rTMS might induce 
alpha suppression whenever the alpha power is high enough $(C)$ while it does not induce any changes whenever alpha power is already low (D) (Zmeykina, unpublished).

Since we found a significant stable effect on phase and power of alpha rhythm induced by $35 \mathrm{mV} / \mathrm{mm}$ rhythmic and arrhythmic rTMS, this intensity was chosen for the second experiment where we stimulated the primary motor cortex (M1) similarly. In this study, in order to access in parallel corticospinal excitability and prove the hypothesis of induced inhibitory circuits, we collected motor evoked potentials from a right-hand muscle in addition to EEG. We reproduced the online synchronization effect from the first experiment. Moreover, we demonstrated the increase of alpha and beta rhythms in the stimulation target (M1) both by rhythmic and arrhythmic rTMS protocols. The power changes were different from the first experiment, where we found only an apparent alpha suppression after arrhythmic rTMS. The concept of entrainment can explain the reason for the contradicting findings. In the presence of noise in the oscillatory system, the phase difference fluctuates randomly when driven by noise or chaotic forces similarly to arrhythmic stimulation; therefore, the induced effect might become unpredictable. In other words, the amplitude of alpha activity is much stronger in the occipital than sensorimotor areas where it is mixed with close by frequency beta rhyhtm, so the high differences in signal to noise ratio between two regions could result in the opposite effect. Therefore, arrhythmic rTMS suppresses initial high amplitude alpha but, on the other hand, increases the amplitude whenever alpha is less pronounced (Figure 21, C-D).

Additionally, we demonstrated the effectiveness of low-intensity rTMS on corticospinal excitability. We demonstrated that increased $\mu$-alpha power changes by rhythmic rTMS resulted in inhibition and decreased motor evoked potentials supporting the inhibition-timing hypothesis of alpha rhythm functions. We also expected that arrhythmic rTMS would desynchronize the alpha rhythm, which would result in excitation and higher MEP amplitudes. Nevertheless, the the exitability changes only by rhythmic but not arrhythmic rTMS may contribute to the role of 
spike-timing-dependent plasticity which appear after synchronous activation of pre and postsynaptic neurons. We assume that arrhythmic rTMS at low intensity could not induce a constant temporal correlation between them; therefore, it could not induce long-lasting changes in the level of cortical excitability.

The effects we observed after rhythmic stimulation are related to the modulation of the ongoing alpha rhythm. It is necessary to notice that we did not observe any TEP even at the highest intensities of ca. $50 \mathrm{mV} / \mathrm{mm}$. Therefore the changes in power induced by rhythmic rTMS are not TMS-locked and mostly related to the power modulation induced by structural mechanisms which are defined as mechanisms changing the parameter of system but not system itself similarly to those that involve voltage dependent receptors, such as NMDA receptors. These receptors do not cause depolarization directly but change the units sensitivity to depolarization ${ }^{64}$. Contrary to strong rTMS applied at conventional intensities of ca. $100 \mathrm{mV} / \mathrm{mm}$, the increased power is not a short-lasting immediate response but might be induced by the autonomous synchronization of local neuronal assemblies.

A

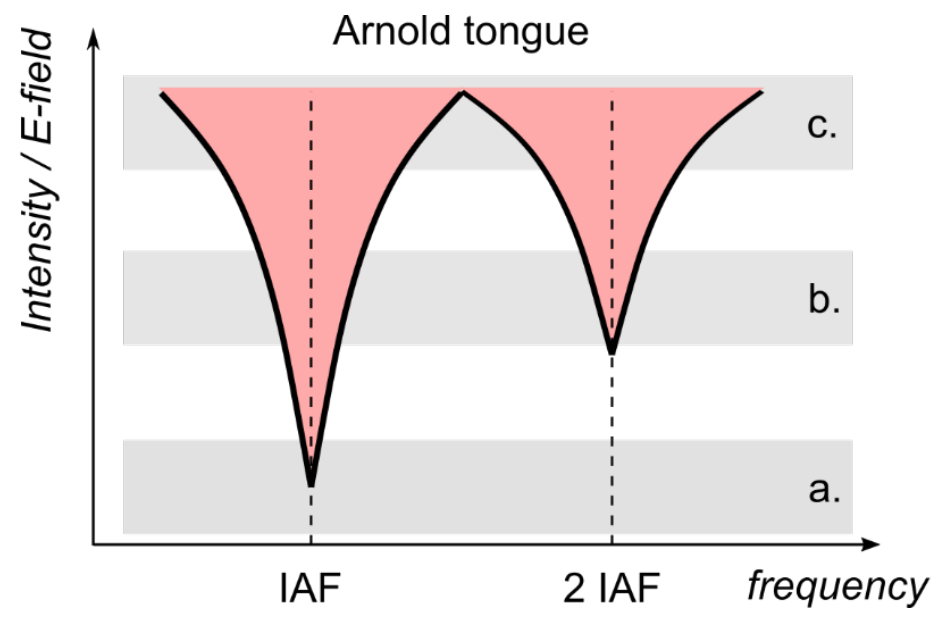

B

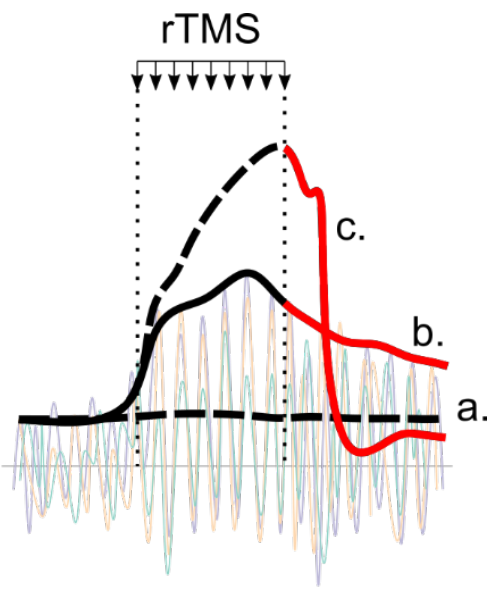

Figure 22. The hypothesis of neural entrainment is based on Arnold tongue model of the oscillatory response to stimulation by exogenous rhythmic EF. The neuronal assemblies might behave in three different scenarios. a. A stimulation of too low intensity cannot interact with the ongoing oscillation and induce phase-locking synchronization. Further power modulation does not occur at any frequencies. Whenever the applied EF strength is too high (c.), the system phase 
resets to each pulse, and the response of rhythmic activity is related to resonance but not neural entrainment. The effect is short-lasting (TMS-locked response duration is less than 1 seconds) and might be confounded with a direct cortical response and other multisensory stimulation effects. There is an optimal region (b.) of rTMS intensities (EF), which might be specific for each region. In the current thesis, $35 \mathrm{mV} / \mathrm{mm}$ was an optimal intensity to induce entrainment effect, which is characterized by immediate synchronization of ongoing rhythm to applied intensity (IAF) and its subharmonic ( 2 IAF) and further induced power changes (unpublished).

\section{Significance and conclusion}

Overall, using rTMS at low intensities and a prospective computational modeling approach has many benefits for research. First, the stimulation itself is well tolerated and does not evoke a strong cranial muscle response, and produces much shorter TMS-induced decay artifacts on EEG. Second, the click produced by TMS devices is much lower, and we believe that it is possible to mask it entirely in the future by improving the audio systems. Using the EF values instead of threshold-based approaches allows comparing the results between participants, and even brain areas and contributing to the understanding of observed neural mechanisms. Moreover, applying low-intensity rTMS might help to overcome coil heating problems and to study more extended rTMS protocols.

In this thesis, the set of experiments in parietal and motor regions confirmed the hypothesis of the low-intensity rTMS induced entrainment effect. We defined the intensity of about 35 $\mathrm{mV} / \mathrm{mm}$ of induced $\mathrm{EF}$ in the target, enough to induce the synchronization and further power modulation associated with corticospinal excitability changes. We also developed and applied a new approach for dosing rTMS intensity based on an individualized adjustment to the target, frequency, and individual anatomy. Based on the results, we specified the entrainment intensity region based on the Arnold tongue model and defined the neural entrainment effect. 


\section{Bibliography}

1. Lefaucheur, J. P. et al. Evidence-based guidelines on the therapeutic use of repetitive transcranial magnetic stimulation (rTMS): An update (2014-2018). Clinical Neurophysiology (2020). doi:10.1016/j.clinph.2019.11.002

2. Rothwell, J. C. Overview of neurophysiology of movement control. Clin. Neurol. Neurosurg. 114, 432-435 (2012).

3. Dayan, E., Censor, N., Buch, E. R., Sandrini, M. \& Cohen, L. G. Noninvasive brain stimulation: From physiology to network dynamics and back. Nature Neuroscience (2013). doi:10.1038/nn.3422

4. Foxe, J. J. \& Snyder, A. C. The role of alpha-band brain oscillations as a sensory suppression mechanism during selective attention. Front. Psychol. (2011). doi:10.3389/fpsyg.2011.00154

5. Misselhorn, J., Friese, U. \& Engel, A. K. Frontal and parietal alpha oscillations reflect attentional modulation of cross-modal matching. Sci. Rep. (2019). doi:10.1038/s41598019-41636-w

6. van Diepen, R. M., Miller, L. M., Mazaheri, A. \& Geng, J. J. The role of alpha activity in spatial and feature- based attention. eNeuro (2016). doi:10.1523/ENEURO.0204-16.2016

7. Park, H. et al. Formation of visual memories controlled by gamma power phase-locked to alpha oscillations. Sci. Rep. 6, 28092 (2016).

8. Saalmann, Y. B., Pinsk, M. A., Wang, L., Li, X. \& Kastner, S. The pulvinar regulates information transmission between cortical areas based on attention demands. Science (80.). (2012). doi:10.1126/science. 1223082

9. Destexhe, A. \& Paré, D. Impact of network activity on the integrative properties of neocortical pyramidal neurons in vivo. J. Neurophysiol. (1999). doi:10.1152/jn.1999.81.4.1531

10. Roelfsema, P. R., Engel, A. K., König, P. \& Singer, W. Visuomotor integration is associated with zero time-lag synchronization among cortical areas. Nature (1997). doi: $10.1038 / 385157 \mathrm{a} 0$

11. Rodriguez, E. et al. Perception's shadow: Long-distance synchronization of human brain activity. Nature (1999). doi:10.1038/17120

12. Buzsáki, G., Anastassiou, C. a \& Koch, C. The origin of extracellular fields and currents-EEG, ECoG, LFP and spikes. Nat. Rev. Neurosci. 13, 407-20 (2012). 
13. Başar, E. A review of alpha activity in integrative brain function: Fundamental physiology, sensory coding, cognition and pathology. Int. J. Psychophysiol. 86, 1-24 (2012).

14. Lopes da Silva, F. Neural mechanisms underlying brain waves: from neural membranes to networks. Electroencephalography and Clinical Neurophysiology (1991). doi:10.1016/0013-4694(91)90044-5

15. Lopes da Silva, F. H., Vos, J. E., Mooibroek, J. \& van Rotterdam, A. Relative contributions of intracortical and thalamo-cortical processes in the generation of alpha rhythms, revealed by partial coherence analysis. Electroencephalogr. Clin. Neurophysiol. (1980). doi:10.1016/0013-4694(80)90011-5

16. Jones, S. R., Pinto, D. J., Kaper, T. J. \& Kopell, N. Alpha-frequency rhythms desynchronize over long cortical distances: A modeling study. J. Comput. Neurosci. (2000). doi:10.1023/A:1026539805445

17. Haegens, S., Cousijn, H., Wallis, G., Harrison, P. J. \& Nobre, A. C. Inter- and intraindividual variability in alpha peak frequency. Neuroimage 92, 46-55 (2014).

18. Haegens, S. et al. Laminar profile and physiology of the $\alpha$ rhythm in primary visual, auditory, and somatosensory regions of neocortex. J. Neurosci. (2015). doi:10.1523/JNEUROSCI.0600-15.2015

19. Halgren, M. et al. The generation and propagation of the human alpha rhythm. Proc. Natl. Acad. Sci. U. S. A. 116, 23772-23782 (2019).

20. Jones, S. R. et al. Cued spatial attention drives functionally relevant modulation of the mu rhythm in primary somatosensory cortex. J. Neurosci. 30, 13760-13765 (2010).

21. Klimesch, W. Alpha-band oscillations, attention, and controlled access to stored information. Trends in Cognitive Sciences (2012). doi:10.1016/j.tics.2012.10.007

22. Pfurtscheller, G. Functional brain imaging based on ERD/ERS. in Vision Research (2001). doi:10.1016/S0042-6989(00)00235-2

23. Eidelman-Rothman, M., Levy, J. \& Feldman, R. Alpha oscillations and their impairment in affective and post-traumatic stress disorders. Neurosci. Biobehav. Rev. 68, 794-815 (2016).

24. Pfurtscheller, G., Stancák, A. \& Neuper, C. Post-movement beta synchronization. A correlate of an idling motor area? Electroencephalogr. Clin. Neurophysiol. (1996). doi:10.1016/0013-4694(95)00258-8

25. Klimesch, W., Sauseng, P. \& Hanslmayr, S. EEG alpha oscillations: The inhibition-timing hypothesis. Brain Res. Rev. 53, 63-88 (2007).

26. Jaworska, N., Blier, P., Fusee, W. \& Knott, V. Alpha power, alpha asymmetry and anterior 
cingulate cortex activity in depressed males and females. J. Psychiatr. Res. (2012). doi:10.1016/j.jpsychires.2012.08.003

27. Dharmadhikari, A., Jaiswal, S., Tandle, A., Sinha, D. \& Jog, N. Study of frontal alpha asymmetry in mild depression: A potential biomarker or not? J. Neurosci. Rural Pract. (2019). doi:10.4103/jnrp.jnrp_293_18

28. Grin-Yatsenko, V. A., Baas, I., Ponomarev, V. A. \& Kropotov, J. D. Independent component approach to the analysis of EEG recordings at early stages of depressive disorders. Clin. Neurophysiol. (2010). doi:10.1016/j.clinph.2009.11.015

29. Clementz, B. A., Sponheim, S. R., Iacono, Wi. G. \& Beiser, M. Resting EEG in firstepisode schizophrenia patients, bipolar psychosis patients, and their first-degree relatives. Psychophysiology (1994). doi:10.1111/j.1469-8986.1994.tb01052.x

30. Karadağ, F. et al. Quantitative EEG analysis in obsessive compulsive disorder. Int. J. Neurosci. (2003). doi:10.1080/00207450390200963

31. Bucci, P. et al. Executive hypercontrol in obsessive-compulsive disorder: Electrophysiological and neuropsychological indices. Clin. Neurophysiol. (2004). doi:10.1016/j.clinph.2003.12.031

32. Jeong, J. EEG dynamics in patients with Alzheimer's disease. Clinical Neurophysiology (2004). doi:10.1016/j.clinph.2004.01.001

33. Adler, G., Brassen, S. \& Jajcevic, A. EEG coherence in Alzheimer's dementia. J. Neural Transm. (2003). doi:10.1007/s00702-003-0024-8

34. Herrmann, C. S. \& Demiralp, T. Human EEG gamma oscillations in neuropsychiatric disorders. Clinical Neurophysiology (2005). doi:10.1016/j.clinph.2005.07.007

35. Gasser, T., Bächer, P. \& Steinberg, H. Test-retest reliability of spectral parameters of the EEG. Electroencephalogr. Clin. Neurophysiol. (1985). doi:10.1016/00134694(85)90005-7

36. Cole, S. \& Voytek, B. Brain oscillations and the importance of waveform shape. Trends Cogn. Sci. 21, 137-149 (2016).

37. Tallon-Baudry, C., Bertrand, O., Delpuech, C. \& Pernier, J. Oscillatory $\gamma$-band (30-70 Hz) activity induced by a visual search task in humans. J. Neurosci. (1997). doi:10.1523/jneurosci.17-02-00722.1997

38. Arkady Pikovsky, Michael Rosenblum, and J. K. Synchronization: A Universal Concept in Nonlinear Sciences. Cambridge U. Press. New York (2001). doi:10.1063/1.1554136

39. Liu, A. et al. Immediate neurophysiological effects of transcranial electrical stimulation. Nat. Commun. (2018). doi:10.1038/s41467-018-07233-7 
40. Thut, G. et al. Rhythmic TMS causes local entrainment of natural oscillatory signatures. Curr. Biol. 21, 1176-1185 (2011).

41. Fröhlich, F. \& McCormick, D. A. Endogenous electric fields may guide neocortical network activity. Neuron 67, 129-143 (2010).

42. Turi, Z. et al. Both the cutaneous sensation and phosphene perception are modulated in a frequency-specific manner during transcranial alternating current stimulation. Restor. Neurol. Neurosci. (2013). doi:10.3233/RNN-120297

43. Opitz, A., Paulus, W., Will, S., Antunes, A. \& Thielscher, A. Determinants of the electric field during transcranial direct current stimulation. Neuroimage 109, 140-150 (2015).

44. Barker, A. T., Jalinous, R. \& Freeston, I. L. Non-invasive magnetic stimulation of human motor cortex. Lancet 325, 1106-1107 (1985).

45. Barker, A. T., Freeston, I. L., Jalinous, R. \& Jarratt, J. A. Magnetic stimulation of the human brain and peripheral nervous system: an introduction and the results of an initial clinical evaluation. Neurosurgery (1987). doi:10.1097/00006123-198701000-00024

46. Fox, P. T. et al. Column-Based Model of Electric Field Excitation of Cerebral Cortex. Hum. Brain Mapp. 22, 1-14 (2004).

47. Deng, Z. De, Lisanby, S. H. \& Peterchev, A. V. Electric field depth-focality tradeoff in transcranial magnetic stimulation: Simulation comparison of 50 coil designs. Brain Stimul. (2013). doi:10.1016/j.brs.2012.02.005

48. Gomez-Tames, J., Hamasaka, A., Laakso, I., Hirata, A. \& Ugawa, Y. Atlas of optimal coil orientation and position for TMS: A computational study. Brain Stimul. 11, 839-848 (2018).

49. Opitz, A., Windhoff, M., Heidemann, R. M., Turner, R. \& Thielscher, A. How the brain tissue shapes the electric field induced by transcranial magnetic stimulation. Neuroimage 58, 849-859 (2011).

50. Thielscher, A., Opitz, A. \& Windhoff, M. Impact of the gyral geometry on the electric field induced by transcranial magnetic stimulation. Neuroimage (2011). doi:10.1016/j.neuroimage.2010.07.061

51. Peterchev, A. V., Murphy, D. L. \& Lisanby, S. H. Repetitive transcranial magnetic stimulator with controllable pulse parameters (cTMS). in 2010 Annual International Conference of the IEEE Engineering in Medicine and Biology Society, EMBC'10 (2010). doi:10.1109/IEMBS.2010.5626287

52. Peterchev, A. V, Dostilio, K., Rothwell, J. C. \& Murphy, D. L. Controllable pulse parameter transcranial magnetic stimulator with enhanced circuit topology and pulse 
shaping. J. Neural Eng. 11, 056023 (2014).

53. Aberra, A. S., Wang, B., Grill, W. M. \& Peterchev, A. V. Simulation of transcranial magnetic stimulation in head model with morphologically-realistic cortical neurons. Brain Stimul. 13, 175-189 (2020).

54. Cappon, D., D’ostilio, K., Garraux, G., Rothwell, J. \& Bisiacchi, P. Effects of $10 \mathrm{~Hz}$ and $20 \mathrm{~Hz}$ Transcranial Alternating Current Stimulation on Automatic Motor Control. Brain Stimul. 9, 518-524 (2016).

55. Ravazzani, P., Ruohonen, J., Grandori, F. \& Tognola, G. Magnetic stimulation of the nervous system: Induced electric field in unbounded, semi-infinite, spherical, and cylindrical media. Ann. Biomed. Eng. (1996). doi:10.1007/BF02684229

56. Salvadora, R., Silvaa, S., Basserb, P. J. \& Miranda, P. C. Determining which mechanisms lead to activation in the motor cortex: a modeling study of transcranial magnetic stimulation using realistic stimulus waveforms and sulcal geometry. Clin Neurophysiol (2011). doi:10.1016/j.clinph.2010.09.022.Determining

57. Weise, K., Numssen, O., Thielscher, A., Hartwigsen, G. \& Knösche, T. R. A novel approach to localize cortical TMS effects. Neuroimage 209, (2020).

58. Rossi, S. et al. Safety and recommendations for TMS use in healthy subjects and patient populations, with updates on training, ethical and regulatory issues: Expert Guidelines. Clin. Neurophysiol. (2020). doi:10.1016/j.clinph.2020.10.003

59. Rosanova, M. et al. Natural Frequencies of Human Corticothalamic Circuits. J. Neurosci. 29, 7679-7685 (2009).

60. Thut, G. \& Miniussi, C. New insights into rhythmic brain activity from TMS-EEG studies. Trends Cogn. Sci. 13, 182-189 (2009).

61. Weisz, N., Lüchinger, C., Thut, G. \& Müller, N. Effects of individual alpha rTMS applied to the auditory cortex and its implications for the treatment of chronic tinnitus. Hum. Brain Mapp. 35, 14-29 (2014).

62. Herring, J. D., Thut, G., Jensen, O. \& Bergmann, T. O. Attention Modulates TMS-Locked Alpha Oscillations in the Visual Cortex. J. Neurosci. 35, 14435-14447 (2015).

63. Hanslmayr, S., Matuschek, J. \& Fellner, M. C. Entrainment of prefrontal beta oscillations induces an endogenous echo and impairs memory formation. Curr. Biol. 24, 904-909 (2014).

64. David, O., Kilner, J. M. \& Friston, K. J. Mechanisms of evoked and induced responses in MEG/EEG. Neuroimage (2006). doi:10.1016/j.neuroimage.2006.02.034

65. Thut, G. et al. Guiding transcranial brain stimulation by EEG/MEG to interact with 
ongoing brain activity and associated functions: A position paper. Clin. Neurophysiol. 128, 843-857 (2017).

66. Nikouline, V., Ruohonen, J. \& Ilmoniemi, R. J. The role of the coil click in TMS assessed with simultaneous EEG. Clin. Neurophysiol. (1999). doi:10.1016/S1388-2457(99)00070$\mathrm{X}$

67. Mutanen, T., Mäki, H. \& Ilmoniemi, R. J. The effect of stimulus parameters on TMS-EEG muscle artifacts. Brain Stimul. 6, 371-376 (2013).

68. Gaetz, W. \& Cheyne, D. Localization of sensorimotor cortical rhythms induced by tactile stimulation using spatially filtered MEG. Neuroimage (2006). doi:10.1016/j.neuroimage.2005.10.009

69. Shitara, H., Shinozaki, T., Takagishi, K., Honda, M. \& Hanakawa, T. Movement and afferent representations in human motor areas: A simultaneous neuroimaging and transcranial magnetic/peripheral nerve-stimulation study. Front. Hum. Neurosci. (2013). doi:10.3389/fnhum.2013.00554

70. Conde, V. et al. The non-transcranial TMS-evoked potential is an inherent source of ambiguity in TMS-EEG studies. Neuroimage 185, 300-312 (2019).

71. Turi, Z., Lenz, M., Paulus, W., Mittner, M. \& Vlachos, A. Selecting stimulation intensity in repetitive transcranial magnetic stimulation studies: A systematic review between 1991 and 2020. bioRxiv 2020.09.28.316190 (2020). doi:10.1101/2020.09.28.316190

72. Zmeykina, E., Mittner, M., Paulus, W. \& Turi, Z. Weak rTMS-induced electric fields produce neural entrainment in humans. Sci. Rep. 10, 1-16 (2020).

73. Zmeykina, E., Mittner, M., Paulus, W. \& Turi, Z. Short-lived alpha power suppression induced by low-intensity arrhythmic rTMS. bioRxiv 2020.10.28.358986 (2020). doi:10.1101/2020.10.28.358986

74. Zmeykina, E., Turi, Z., Antal, A. \& Paulus, W. Inhibiting corticospinal excitability by entraining ongoing $\mu$-alpha rhythm in motor cortex. 2507, 1-9 (2021).

75. Buzsáki, G., Logothetis, N. \& Singer, W. Scaling brain size, keeping timing: Evolutionary preservation of brain rhythms. Neuron (2013). doi:10.1016/j.neuron.2013.10.002

76. Watson, B. O. \& Buzsáki, G. Sleep, Memory \& Brain Rhythms. Daedalus 144, 67-82 (2015).

77. Rabiller, G., He, J. W., Nishijima, Y., Wong, A. \& Liu, J. Perturbation of brain oscillations after ischemic stroke: A potential biomarker for post-stroke function and therapy. Int. J. Mol. Sci. 16, 25605-25640 (2015).

78. Anastassiou, C. A. \& Koch, C. Ephaptic coupling to endogenous electric field activity: 
Why bother? Curr. Opin. Neurobiol. 31, 95-103 (2015).

79. Bergmann, T. O., Karabanov, A., Hartwigsen, G., Thielscher, A. \& Siebner, H. R. Combining non-invasive transcranial brain stimulation with neuroimaging and electrophysiology: Current approaches and future perspectives. Neuroimage 140, 4-19 (2016).

80. Fröhlich, F. Experiments and models of cortical oscillations as a target for noninvasive brain stimulation. Prog. Brain Res. 222, 41-73 (2015).

81. Paulus, W., Peterchev, A. V. \& Ridding, M. Transcranial electric and magnetic stimulation: technique and paradigms. Handb. Clin. Neurol. 116, 329-342 (2013).

82. Opitz, A. et al. Is sham cTBS real cTBS? The effect on EEG dynamics. Front. Hum. Neurosci. 8, 1043 (2014).

83. Sahlsten, H. et al. Electric field navigated transcranial magnetic stimulation for chronic tinnitus: A pilot study Electric fi eld navigated transcranial magnetic stimulation for chronic tinnitus: A pilot study. 2027, (2015).

84. Hanslmayr, S., Matuschek, J. \& Fellner, M. C. Entrainment of prefrontal beta oscillations induces an endogenous echo and impairs memory formation. Curr. Biol. 24, 904-909 (2014).

85. Romei, V. et al. Causal evidence that intrinsic beta-frequency is relevant for enhanced signal propagation in the motor system as shown through rhythmic TMS. Neuroimage 126, 120-130 (2016).

86. Albouy, P., Weiss, A., Baillet, S. \& Zatorre, R. J. Selective Entrainment of Theta Oscillations in the Dorsal Stream Causally Enhances Auditory Working Memory Performance. Neuron 94, 193-206.e5 (2017).

87. Krause, M. R. et al. Transcranial Direct Current Stimulation Facilitates Associative Learning and Alters Functional Connectivity in the Primate Brain Article Transcranial Direct Current Stimulation Facilitates Associative Learning and Alters Functional Connectivity in the Pri. Curr. Biol. 27, 3086-3096 (2017).

88. Ozen, S. et al. Transcranial Electric Stimulation Entrains Cortical Neuronal Populations in Rats. J. Neurosci. 30, 11476-11485 (2010).

89. Krause, M. R., Vieira, P. G., Csorba, B. A., Pilly, P. K. \& Pack, C. C. Transcranial alternating current stimulation entrains single-neuron activity in the primate brain. 1-9 (2019). doi:10.1073/pnas.1815958116

90. Thut, G. et al. Rhythmic TMS causes local entrainment of natural oscillatory signatures. Curr. Biol. 21, 1176-1185 (2011). 
91. Jean-Philippe Lachaux, Eugenio Rodriguez, Jacques Martinerie, and F. J. V. Measuring Phase Synchrony in Brain Signals. Hum. Brain Mapp. (1999). doi:10.1017/S0007680500048066

92. Glass, L. Synchronization and rhythmic processes in physiology. Naure 410, 277-284 (2001).

93. Romei, V. et al. Causal evidence that intrinsic beta-frequency is relevant for enhanced signal propagation in the motor system as shown through rhythmic TMS. Neuroimage 126, 120-130 (2016).

94. Romei, V., Gross, J. \& Thut, G. Report Sounds Reset Rhythms of Visual Cortex and Corresponding Human Visual Perception. Curr. Biol. 22, 807-813 (2012).

95. Wu, W. et al. RESEARCH ARTICLE ARTIST: A fully automated artifact rejection algorithm for single-pulse TMS-EEG data. 1607-1625 (2018). doi:10.1002/hbm.23938

96. Yuan, H., Shou, G., Gleghorn, D., Ding, L. \& Cha, Y. Resting State Functional Connectivity Signature of Treatment Effects of Repetitive Transcranial Magnetic Stimulation in Mal de Debarquement Syndrome. Brain Connect. 7, 617-626 (2017).

97. Vöröslakos, M. et al. Direct effects of transcranial electric stimulation on brain circuits in rats and humans. Nat. Commun. 9, 483 (2018).

98. Peterchev, A. V., Goetz, S. M., Westin, G. G., Luber, B. \& Lisanby, S. H. Pulse width dependence of motor threshold and input - output curve characterized with controllable pulse parameter transcranial magnetic stimulation. Clin. Neurophysiol. 124, 1364-1372 (2013).

99. Hallett, M. Transcranial Magnetic Stimulation : A Primer. Neuron 55, 187-199 (2007).

100. Rossi, S., Hallett, M., Rossini, P. M., Pascual-Leone, A. \& Safety of TMS Consensus Group, T. S. of T. C. Safety, ethical considerations, and application guidelines for the use of transcranial magnetic stimulation in clinical practice and research. Clin. Neurophysiol. 120, 2008-39 (2009).

101. Di Lazzaro, V. ., Oliviero, A., Pilato, F., Saturno, E. \& Dileone, M. The physiological basis of transcranial motor cortex stimulation in conscious humans. Clin. Neurophysiol. 115, 255-266 (2004).

102. Epstein, C. M. et al. Localization and characterization of speech arrest during transcranial magnetic stimulation. Clin. Neurophysiol. 110, 1073-1079 (1999).

103. Berényi, A., Belluscio, M., Mao, D. \& Buzsáki, G. Closed-loop control of epilepsy by transcranial electrical stimulation. Science (80-. ). 337, 735-7 (2012).

104. Huang, Y. \& Parra, L. C. Can transcranial electric stimulation with multiple electrodes 
reach deep targets ? Brain Stimul. 12, 30-40 (2019).

105. Bungert, A., Antunes, A., Espenhahn, S. \& Thielscher, A. Where does TMS Stimulate the Motor Cortex? Combining Electrophysiological Measurements and Realistic Field Estimates to Reveal the Affected Cortex Position. Cereb. Cortex 27, 5083-5094 (2017).

106. Laakso, I., Murakami, T., Hirata, A. \& Ugawa, Y. Where and what TMS activates: Experiments and modeling. Brain Stimul. 11, 166-174 (2018).

107. Laakso, I., Mikkonen, M., Koyama, S., Hirata, A. \& Tanaka, S. Can electric fields explain inter- individual variability in transcranial direct current stimulation of the motor cortex ? Sci. Rep. 9, 626 (2019).

108. Ziemann, U. Thirty years of transcranial magnetic stimulation: where do we stand? Exp. Brain Res. 235, 973-984 (2017).

109. Huang, Y.-Z. et al. Plasticity induced by non-invasive transcranial brain stimulation : A position paper. Clin. Neurophysiol. 128, 2318-2329 (2017).

110. Lefaucheur, J. P. et al. Evidence-based guidelines on the therapeutic use of repetitive transcranial magnetic stimulation (rTMS). Clin. Neurophysiol. 125, 2150-2206 (2014).

111. Nielsen, J. D. et al. Automatic skull segmentation from MR images for realistic volume conductor models of the head : Assessment of the state-of-the-art. Neuroimage 174, 587598 (2018).

112. Vorwerk, J., Oostenveld, R., Piastra, M. C., Magyari, L. \& Wolters, C. H. The FieldTrip - SimBio pipeline for EEG forward solutions. Biomed. Eng. Online 17, 37 (2018).

113. Saturnino, G. B., Thielscher, A., Madsen, K. H., Kn, T. R. \& Weise, K. A principled approach to conductivity uncertainty analysis in electric fi eld calculations. Neuroimage 188, 821-834 (2019).

114. Rimpil, V., Koulouri, A., Lucka, F., Kaipio, J. P. \& Wolters, C. H. Improved EEG source localization with Bayesian uncertainty modelling of unknown skull conductivity. Neuroimage 188, 252-260 (2019).

115. Moliadze, V., Atalay, D., Antal, A. \& Paulus, W. Close to threshold transcranial electrical stimulation preferentially activates inhibitory networks before switching to excitation with higher intensities. Brain Stimul. 5, 505-511 (2012).

116. Lenz, M., Galanis, C., Mu, F., Opitz, A. \& Wierenga, C. J. Repetitive magnetic stimulation induces plasticity of inhibitory synapses. Nat. Commun. 7:10020 (2016). doi:10.1038/ncomms 10020

117. Klimesch, W., Sauseng, P. \& Hanslmayr, S. EEG alpha oscillations: The inhibition-timing 
hypothesis. Brain Res. Rev. 53, 63-88 (2007).

118. Mazaheri, A. \& Jensen, O. Asymmetric amplitude modulations of brain oscillations generate slow evoked responses. J. Neurosci. 28, 7781-7787 (2008).

119. Romei, V., Thut, G., Mok, R. M., Schyns, P. G. \& Driver, J. Causal implication by rhythmic transcranial magnetic stimulation of alpha frequency in feature-based local vs . global attention. Eur. J. Neurosci. 35, 968-974 (2012).

120. Oldfield, R. C. The assessment and analysis of handedness: The Edinburgh inventory. Neuropsychologia 9, 97-113 (1971).

121. Conforto, A. B., Z’Graggen, W. ., Kohl, A. S., Rösler, K. M. \& Kaelin-Lang, A. Impact of coil position and electrophysiological monitoring on determination of motor thresholds to transcranial magnetic stimulation. Clin. Neurophysiol. 115, 812-819 (2004).

122. Thielscher, A., Antunes, A. \& Saturnino, G. B. Field modeling for transcranial magnetic stimulation: A useful tool to understand the physiological effects of TMS? Proc. Annu. Int. Conf. IEEE Eng. Med. Biol. Soc. EMBS 222-225 (2015). doi:10.1109/EMBC.2015.7318340

123. Windhoff, M., Opitz, A. \& Thielscher, A. Electric field calculations in brain stimulation based on finite elements: An optimized processing pipeline for the generation and usage of accurate individual head models. Hum. Brain Mapp. 34, 923-935 (2013).

124. Opitz, A., Windhoff, M., Heidemann, R. M., Turner, R. \& Thielscher, A. How the brain tissue shapes the electric field induced by transcranial magnetic stimulation. Neuroimage 58, 849-859 (2011).

125. Rahman, A. et al. Cellular effects of acute direct current stimulation : somatic and synaptic terminal effects. Jounral Physiol. 10, 2563-2578 (2013).

126. Oostenveld, R., Fries, P., Maris, E. \& Schoffelen, J. M. FieldTrip: Open source software for advanced analysis of MEG, EEG, and invasive electrophysiological data. Comput. Intell. Neurosci. 2011, (2011).

127. Peirce, J. W. PsychoPy-Psychophysics software in Python. J. Neurosci. Methods 162, 813 (2007).

128. Peirce, J. W. Generating stimuli for neuroscience using PsychoPy. Front. Neuroinform. 2, 1-8 (2009).

129. Nikulin, V. V., Kicl̈ic, D., Kähkönen, S. \& Ilmoniemi, R. J. Modulation of electroencephalographic responses to transcranial magnetic stimulation: evidence for changes in cortical excitability related to movement. Eur. J. Neurosci. 18, 1206-1212 (2003). 
130. Lachaux, J. P., Rodriguez, E., Martinerie, J. \& Varela, F. J. Measuring phase synchrony in brain signals. Hum. Brain Mapp. 8, 194-208 (1999).

131. Ando, A. et al. Effects of repetitive transcranial magnetic stimulation ( rTMS ) on attribution of movement to ambiguous stimuli and EEG mu suppression. Brain Res. 1680, 69-76 (2018).

132. Bai, Y. et al. Evaluating the Effect of Repetitive Transcranial Magnetic Stimulation on Disorders of Consciousness by Using. Front. Neurosci. 10, 437 (2016).

133. Bharath, R. D. et al. A Single Session of rTMS Enhances Small-Worldness in Writer' s Cramp : Evidence from Simultaneous. Front. Hum. Neurosci. 11, 443 (2017).

134. Cao, D., Li, Y., Niznikiewicz, M. A. \& Tang, Y. The theta burst transcranial magnetic stimulation over the right PFC a ff ects electroencephalogram oscillation during emotional processing. Prog. Neuropsychopharmacol. Biol. Psychiatry 82, 21-30 (2018).

135. Capotosto, P. et al. Task and Regions Speci fi c Top-Down Modulation of Alpha Rhythms in Parietal Cortex. Cereb. Cortex 27, 4815-4822 (2017).

136. Cha, Y. H. et al. Electrophysiological Signatures of Intrinsic Functional Connectivity Related to rTMS Treatment for Mal de Debarquement Syndrome. Brain Topogr. 31, 1047-1058 (2018).

137. Chen, Y. et al. Assessing rTMS Effects in MdDS : Cross - modal Comparison between Resting State EEG and fMRI Connectivity. Conf. Proc. 39th Annu. Int. Conf. IEEE Eng. Med. Biol. Soc. 1950-1953 (2017). doi:10.1109/EMBC.2017.8037231

138. D’Agata, F. et al. Cognitive and Neurophysiological Effects of Non-invasive Brain Stimulation in Stroke Patients after Motor Rehabilitation. Front. Behav. Neurosci. 10, 135 (2016).

139. Daltrozzo, J., Kotchoubey, B., Gueler, F. \& Karim, A. A. Effects of Transcranial Magnetic Stimulation on Body Perception : No Evidence for Specificity of the Right TemporoParietal Junction. Brain Topogr. 29, 704-715 (2016).

140. Del Felice, A. et al. Neurophysiological, psychological and behavioural correlates of rTMS treatment in alcohol dependence. Drug Alcohol Depend. 158, 147-153 (2016).

141. Di Giacomo, J. et al. Neuroscience Letters Repetitive Transcranial Magnetic Stimulation changes absolute theta power during cognitive / motor tasks. Neurosci. Lett. 687, 77-81 (2018).

142. Emrich, S. M., Johnson, J. S., Sutterer, D. W. \& Postle, B. R. Comparing the Effects of 10-Hz Repetitive TMS on Tasks of Visual STM and Attention. J. Cogn. Neurosci. 29, 286-97 (2017). 
143. Fisher, R. et al. Epilepsy \& Behavior Case Reports Repetitive transcranial magnetic stimulation directed to a seizure focus localized by high-density EEG : A case report. Epilepsy Behav. Case Reports 10, 47-53 (2018).

144. Gongora, M. et al. Low-frequency rTMS over the Parieto - frontal network during a sensorimotor task: The role of absolute beta power in the sensorimotor integration. Neurosci. Lett. 611, 1-5 (2016).

145. He, F. et al. Clinical Study Effects of $20 \mathrm{~Hz}$ Repetitive Transcranial Magnetic Stimulation on Disorders of Consciousness : A Resting-State Electroencephalography Study. Neural Plast. 5036184 (2018).

146. Hunter, A. M. et al. Change in Quantitative EEG Theta Cordance as a Potential Predictor of Repetitive Transcranial Magnetic Stimulation Clinical Outcome in Major Depressive Disorder. Clin. EEG Neurosci. EEG Neurosci 49, 306-315 (2018).

147. Jin, J.-N. et al. The Effects of rTMS Combined with Motor Training on Functional Connectivity in Alpha Frequency Band. Front. Behav. Neurosci. 11, 234 (2017).

148. Kamp, D. et al. High frequency repetitive transcranial magnetic stimulation ( rTMS ) reduces EEG-hypofrontality in patients with schizophrenia. Psychiatry Res. 236, 199-201 (2016).

149. Karton, I. \& Bachmann, T. Disrupting dorsolateral prefrontal cortex by rTMS reduces the P300 based marker of deception. Brain Behav. 7, e00656 (2017).

150. Kazemi, R. et al. Electrophysiological correlates of bilateral and unilateral repetitive transcranial magnetic stimulation in patients with bipolar depression. Psychiatry Res. 240, 364-375 (2016).

151. Kazemi, R. et al. Bilateral Transcranial Magnetic Stimulation on DLPFC Changes Resting State Networks and Cognitive Function in Patients With Bipolar Depression. Front. Hum. Neurosci. 12, 365 (2018).

152. Keuper, K., Terrighena, E. L., Chan, C. C. H. \& Junghoefer, M. How the Dorsolateral Prefrontal Cortex Controls Affective Processing in Absence of Visual Awareness Insights From a Combined EEG-rTMS Study. Front. Hum. Neurosci. 12, 1-19 (2018).

153. Kim, Y. I., Kim, S. M., Kim, H. \& Han, D. H. The Effect of High-Frequency Repetitive Transcranial Magnetic Stimulation on Occupational Stress among Health Care Workers : A Pilot Study. Psychiatry Investig. 13, 622-629 (2016).

154. Kito, S. et al. Transcranial Magnetic Stimulation Modulates Resting EEG Functional Connectivity Between the Left Dorsolateral Prefrontal Cortex and Limbic Regions in Medicated Patients With Treatment-Resistant Depression. Jounal Neuropsychiatry Clin. 
Neurosci. 29, 155-159 (2017).

155. Koch, G. et al. Transcranial magnetic stimulation of the precuneus enhances memory and neural activity in prodromal Alzheimer's s disease. Neuroimage 169, 302-311 (2018).

156. Li, C.-T. et al. Cognition-Modulated Frontal Activity in Prediction and Augmentation of Antidepressant Ef fi cacy : A Randomized Controlled Pilot Study. Cereb. Cortex 26, 202210 (2016).

157. Li, S. et al. Theta and Alpha Oscillations during the Retention Period of Working Memory by rTMS Stimulating the Parietal Lobe. Front. Behav. Neurosci. 11, 170 (2017).

158. Li, Y. et al. The effects of high-frequency rTMS over the left DLPFC on cognitive control in young healthy participants. PLoS One 12, e0179430 (2017).

159. Lowe, C. J., Staines, W. R., Manocchio, F. \& Hall, P. A. The neurocognitive mechanisms underlying food cravings and snack food consumption . A combined continuous theta burst stimulation ( cTBS ) and EEG study. Neuroimage 177, 45-58 (2018).

160. Lozeron, P. et al. Inhibitory rTMS applied on somatosensory cortex in Wilson's disease patients with hand dystonia. J. Neural Transm. 124, 1161-1170 (2017).

161. Möbius, M. et al. Repetitive transcranial magnetic stimulation modulates the impact of a negative mood induction. Soc. Cogn. Affect. Neurosci. 12, 526-533 (2017).

162. Nathou, C. et al. E ff ects of low- and high-frequency repetitive transcranial magnetic stimulation on long-latency auditory evoked potentials. Neurosci. Lett. 686, 198-204 (2018).

163. Nicolo, P., Fargier, R., Laganaro, M. \& Guggisberg, A. G. Neurobiological Correlates of Inhibition of the Right Broca Homolog during New-Word Learning. Front. Hum. Neurosci. 10, 371 (2016).

164. Noda, Y., Zomorrodi, R., Daskalakis, J., Blumberger, D. M. \& Nakamura, M. Enhanced theta-gamma coupling associated with hippocampal volume increase following highfrequency left prefrontal repetitive transcranial magnetic stimulation in patients with major depression. Int. J. Psychophysiol. 133, 169-174 (2018).

165. Oshima, H. et al. Alteration of Duration Mismatch Negativity Induced by Transcranial Magnetic Stimulation Over the Left Parietal Lobe. Clin. EEG Neurosci. 48, 11-19 (2017).

166. Prashad, S., Dedrick, E. S., To, W. T., Vanneste, S. \& Filbey, F. M. Testing the role of the posterior cingulate cortex in processing salient stimuli in cannabis users : an rTMS study. Eur. J. Neurosci. 1-13 (2018). doi:10.1111/ejn.14194

167. Rocha, K. et al. Low-frequency rTMS stimulation over superior parietal cortex medially improves time reproduction and increases the right dorsolateral prefrontal cortex 
predominance. Int. J. Neurosci. 129, 523-533 (2018).

168. Rousseau, E., Melo-silva, C. A., Gakwaya, S. \& Sériès, F. Effects of repetitive transcranial magnetic stimulation of upper airway muscles during sleep in obstructive sleep apnea patients. J. Appl. Physiol. 121, 1217-1225 (2016).

169. Shalbaf, R. et al. Non-linear Entropy Analysis in EEG to Predict Treatment Response to Repetitive Transcranial Magnetic Stimulation in Depression. Front. Pharmacol. 9, 1188 (2018).

170. Shields, J., Devier, D. \& Foundas, A. Journal of the Neurological Sciences Unilateral repetitive transcranial magnetic stimulation di ff erentially affects younger and older adults completing a verbal working memory task. J. Neurol. Sci. 384, 15-20 (2018).

171. Spadone, S., Sestieri, C., Baldassarre, A. \& Capotosto, P. Temporal dynamics of TMS interference over preparatory alpha activity during semantic decisions. Scient 7, 2372 (2017).

172. Tikka, S. K. et al. Safety and Efficacy of Adjunctive $\Theta$ Burst Repetitive Transcranial Magnetic Stimulation to Right Inferior Parietal Lobule in Schizophrenia Patients With A Pilot, Exploratory Study. J. ECT 33, 43-51 (2017).

173. Valiulis, V., Gerulskis, G., Dapšys, K. \& Valavičiūtè, K. The use of MR - less MNI based neuronavigation for $10 \mathrm{~Hz}$ rTMS depression therapy: electrophysiological and clinical implications. Acta Neurobiol. Exp. (Wars). 78, 271-280 (2018).

174. Xia, X. et al. Long-lasting repetitive transcranial magnetic stimulation modulates electroencephalography oscillation in patients with disorders of consciousness. Neuroreport 28, 1022-1029 (2017).

175. Anastassiou, C. A., Perin, R., Markram, H. \& Koch, C. Ephaptic coupling of cortical neurons. Nat. Neurosci. 14, 217-223 (2011).

176. Lenz, M. \& Vlachos, A. Releasing the Cortical Brake by Non-Invasive Electromagnetic Stimulation? rTMS Induces LTD of GABAergic Neurotransmission. Front. Neural Circuits 10, 96 (2016).

177. Ilmoniemi, R. J. et al. Dealing with artifacts in TMS-evoked EEG. Proc. Annu. Int. Conf. IEEE Eng. Med. Biol. Soc. 230-233 (2015). doi:10.1109/EMBC.2015.7318342

178. Thut, G. \& Pascual-Leone, A. A Review of Combined TMS-EEG Studies to Characterize Lasting Effects of Repetitive TMS and Assess Their Usefulness in Cognitive and Clinical Neuroscience. Brain Topogr. 22, 219-232 (2010).

179. Hanslmayr, S., Matuschek, J. \& Fellner, M. C. Entrainment of prefrontal beta oscillations 
induces an endogenous echo and impairs memory formation. Curr. Biol. 24, 904-909 (2014).

180. Thut, G., Schyns, P. G. \& Gross, J. Entrainment of perceptually relevant brain oscillations by non - invasive rhythmic stimulation of the human brain. Front. Psychol. 2, 170 (2011).

181. Puzzo, I., Cooper, N. R., Cantarella, S., Fitzgerald, P. B. \& Russo, R. The effect of rTMS over the inferior parietal lobule on EEG sensorimotor reactivity differs according to selfreported traits of autism in typically developing individuals \$. Brain Res. 1541, 33-41 (2013).

182. Woźniak-Kwaśniewska, A., Szekely, D., Aussedat, P., Bougerol, T. \& David, O. Changes of oscillatory brain activity induced by repetitive transcranial magnetic stimulation of the left dorsolateral prefrontal cortex in healthy subjects. Neuroimage 88, 91-99 (2014).

183. Valiulis, V., Gerulskis, G., Dapšys, K., Vištartaitė, G. \& Šiurkutè, A. Electrophysiological differences between high and low frequency rTMS protocols in depression treatment. Acta Neurobiol. Exp. (Wars). 72, 283-295 (2012).

184. Narushima, K., Mccormick, L. M., Yamada, T., Thatcher, R. W. \& Robinson, R. G. Subgenual Cingulate Theta Activity Predicts Treatment Response of Repetitive Transcranial Magnetic Stimulation in Participants With Vascular Depression. $J$ Neuropsychiatry Clin Neurosci 22, 75-84 (2010).

185. Arai, N. et al. Differences in after-effect between monophasic and biphasic highfrequency rTMS of the human motor cortex. Clin. Neurophysiol. 118, 2227-2233 (2007).

186. Charles E. Schroeder and Peter Lakatos. Low-frequency neuronal oscillations as instruments of sensory selection. Trends Neurosci. 32, 1-16 (2009).

187. Thies, M., Zrenner, C., Ziemann, U. \& Bergmann, T. O. Sensorimotor $\mu$-alpha power is positively related to corticospinal excitability. Brain Stimul. 11, 1119-1122 (2018).

188. Zrenner, C., Desideri, D., Belardinelli, P. \& Ziemann, U. Real-time EEG-defined excitability states determine efficacy of TMS-induced plasticity in human motor cortex. Brain Stimul. 11, 374-389 (2018).

189. Hummel, F., Andres, F., Altenmüller, E., Dichgans, J. \& Gerloff, C. Inhibitory control of acquired motor programmes in the human brain. Brain 125, 404-420 (2002).

190. Pascual-leone, A., Valls-solé, J., Wassermann, E. M. \& Hallett, M. Responses to rapidrate transcranial magnetic stimulation of the human motor cortex. Brain 117, 847-858 (1994).

191. Ogata, K., Nakazono, H., Uehara, T. \& Tobimatsu, S. Brain Stimulation Prestimulus 
cortical EEG oscillations can predict the excitability of the primary motor cortex. Brain Stimul. 12, 1508-1516 (2020).

192. Ploner, M., Gross, J., Timmermann, L., Pollok, B. \& Schnitzler, A. Oscillatory activity reflects the excitability of the human somatosensory system. Neuroimage 32, 1231-1236 (2006).

193. Jung, S. H., Shin, J. E., Jeong, Y. S. \& Shin, H. I. Changes in motor cortical excitability induced by high-frequency repetitive transcranial magnetic stimulation of different stimulation durations. Clin. Neurophysiol. 119, 71-79 (2008).

194. Wagner, T. A., Zahn, M., Grodzinsky, A. J. \& Pascual-Leone, A. Three-dimensional head model simulation of transcranial magnetic stimulation. IEEE Trans. Biomed. Eng. (2004). doi:10.1109/TBME.2004.827925

195. RStudio Team. RStudio: Integrated Development Environment for R. (2020).

196. R Core Team. R: A Language and Environment for Statistical Computing. (2020).

197. Kuznetsova, A., Brockhoff, P. B. \& Christensen, R. H. B. lmerTest Package: Tests in Linear Mixed Effects Models. J. Stat. Softw. 82, 1-26 (2017).

198. Van Veen, B. D., Van Drongelen, W., Yuchtman, M. \& Suzuki, A. Localization of brain electrical activity via linearly constrained minimum variance spatial filtering. IEEE Trans. Biomed. Eng. 44, 867-880 (1997).

199. Oosterom, T. O. and A. van. The potential distribution generated by surface electrodes in inhomogeneous volume conductors of arbitrary shape. IEEE Trans. Biomed. Eng. 38, (1991).

200. Lowet, E., Roberts, M. J., Bonizzi, P., Karel, J. \& De Weerd, P. Quantifying neural oscillatory synchronization: A comparison between spectral coherence and phase-locking value approaches. PLoS One 11, (2016).

201. Azila Noh, N. \& Fuggetta, G. Human cortical theta reactivity to high-frequency repetitive transcranial magnetic stimulation. Hum. Brain Mapp. 33, 2224-2237 (2012).

202. Fuggetta, G., Pavone, E. F., Fiaschi, A. \& Manganotti, P. Acute modulation of cortical oscillatory activities during short trains of high-frequency repetitive transcranial magnetic stimulation of the human motor cortex: A combined EEG and TMS study. Hum. Brain Mapp. 29, 1-13 (2008).

203. Lorenzano, C. et al. Spread of electrical activity at cortical level after repetitive magnetic stimulation in normal subjects. Exp. Brain Res. 147, 186-192 (2002).

204. Modugno, N. et al. The prolonged cortical silent period in patients with Huntington's disease. Clin. Neurophysiol. (2001). doi:10.1016/S1388-2457(01)00599-5 
205. Maeda, F., Keenan, J. P., Tormos, J. M., Topka, H. \& Pascual-Leone, A. Modulation of corticospinal excitability by repetitive transcranial magnetic stimulation. Clin. Neurophysiol. (2000). doi:10.1016/S1388-2457(99)00323-5

206. Daskalakis, Z. J. et al. The effects of repetitive transcranial magnetic stimulation on cortical inhibition in healthy human subjects. Exp. Brain Res. (2006). doi:10.1007/s00221006-0472-0

207. Mäki, H. \& Ilmoniemi, R. J. EEG oscillations and magnetically evoked motor potentials reflect motor system excitability in overlapping neuronal populations. Clin. Neurophysiol. (2010). doi:10.1016/j.clinph.2009.11.078

208. Desideri, D., Zrenner, C., Gordon, P. C., Id, U. Z. \& Belardinelli, P. Nil effects of $\mu$ rhythm phase-dependent burst-rTMS on cortical excitability in humans : A resting-state EEG and TMS-EEG study. 1-17 (2018).

209. Lenz, M. et al. Repetitive magnetic stimulation induces plasticity of inhibitory synapses. Nat. Commun. 7, (2016). 


\section{List of figures}

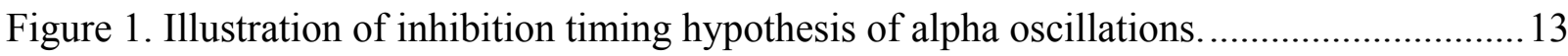

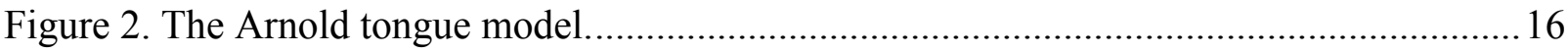

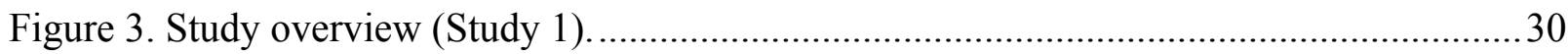

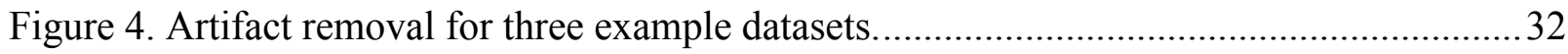

Figure 5. Rhythmic rTMS synchronized ongoing posterior alpha rhythms indicated by increased phase locking values

Figure 6. Preprocessing pipeline does not artificially increase the degree of neural synchronization 36

Figure 7. Increased and sustained neural synchronization during rhythmic but not during arrhythmic or sham rTMS. .38

Figure 8. Group-level mean and median values of the EF for parietal and occipital ROIs........40

Figure 9. Our prospectively determined rTMS settings fell in the range of $17-42 \%$ of RMT. ..42

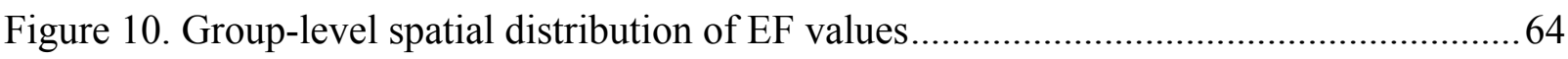

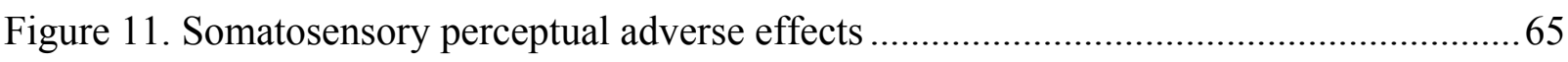

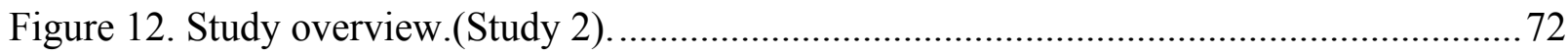

Figure 13. Alpha power change after the rTMS bursts compared with the baseline..................76

Figure 14. Real rTMS did not change the spectral power relative to the sham rTMS at IAF....77

Figure 15. Lack of significant differences in the individual alpha power between rhythmic and arrhythmic rTMS.

Figure 16. Schematic of study flow (Study 3)

Figure 17. Rhythmic rTMS synchronized ongoing $\mu$-alpha rhythms indicated by increased PLVs near the stimulation target.....

Figure 18. Active stimulation protocols increased power in $\mu$-alpha and beta frequency ranges immediately after stimulation

Figure 19. Rhythmic rTMS protocol increased local $\mu$-alpha and beta power at the stimulation target.

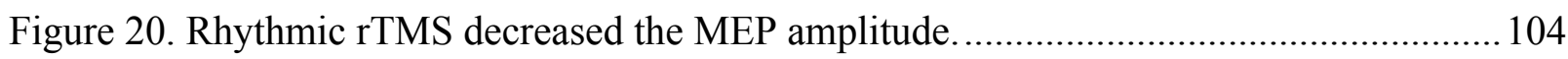

Figure 21. Illustration of entrainment effect induced by $35 \mathrm{mV} / \mathrm{mm}$ rTMS. 102

Figure 22. The hypothesis of neural entrainment based on Arnold tongue model of the oscillatory response to stimulation by exogenous rhythmic EF. 


\section{List of tables}

Table 1. The participant information in the main and control experiments..............................48

Table 2. The five tissue compartments of the head model and their conductivity values ..........54

Table 3. The vast majority of rTMS studies determine stimulation intensity with the so-called

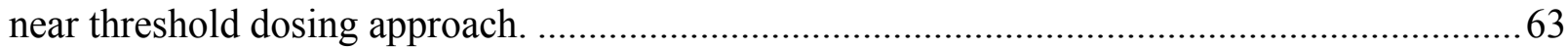

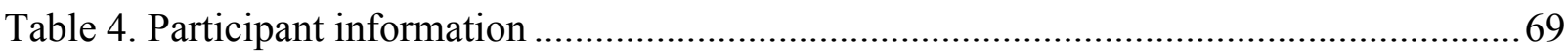

Table 5. Summary of studies investigating the rTMS-induced electrophysiological aftereffects 83

Table 6. Overview of rTMS stimulation conditions and stimulation parameters......................92

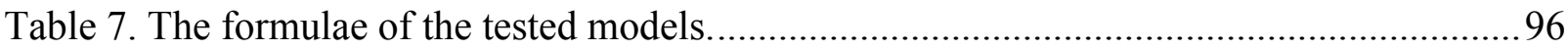




\section{Acknowledgments}

I am very grateful to my supervisor, Prof. Walter Paulus, for highly qualified support, guidance, advice, and the sourcing of research funding under his department that facilitated this study. He gave a lot of critical but always motivating comments and discussions at all stages of this work. Apart from that, many appreciations go to Dr. Zsolt Turi for scientific inspiration and his engagement to the project at all stages from conceptualization until manuscript preparation.

The colleagues in the institute are also acknowledged as they were so supportive in terms of encouragement and shaping my thoughts: Albert, Alexandra, Prof. Andrea Antal, Annika, Christina, Daniel, Ivan, Faizal, Gabriel, Prof. Martin Sommer, and many other colleagues and students who visited us shortly. I thank all of you and appreciate everything shown by everybody in our group at different times.

I highly appreciate the moral support from my family members and friends both in Russia and Germany. They have been supportive since I started my academic journey. Their positive feedback and kind words were incredibly precious when staying abroad.

Last but not least, I am also grateful to the staff of GGNB and my program for accepting me and for the academic and financial support. This work was partly supported by the Ph.D. program "Theoretical and Computational Neuroscience" at the Georg August University Göttingen. 


\section{Declaration of contribution}

\section{Statement of Originality}

I hereby declare that this thesis has been written independently with no other sources and aids quoted in the text, references, and acknowledgments.

Göttingen, 08.02.2021

Elina Zmeykina 


\section{Curriculum Vitae}

Elina Zmeykina,

28 years old, Russia

Address: $\quad$ Göttingen, Germany

Email: $\quad$ elina.zmeykina@gmail.com;

Telephone: $\quad$ +4901741722618

\section{Education}

01/2017 - Present

\section{Ph.D. Candidate | Georg-August University Göttingen, Germany}

- Program of theoretical and computational neuroscience

- Thesis Title: 'Optimizing stimulation parameters for manipulating neuronal communication by using low-intensity repetitive transcranial magnetic stimulation'.

- Supervisor: Prof. Walter Paulus

$09 / 2008-02 / 2014$

Engineer physicist | National Research Nuclear University 'MEPhI (Moscow Engineer Physics Institute)', Russia

- Diploma in Human and Environmental Radiation Safety (Biophysics)

- Thesis Title: 'The effects of transcranial magnetic stimulation on the human brain resting-state networks'

- Supervisor: Dr. Alexander Chervyakov

\section{Teaching experience}

- Workshop of advanced TMS in research (University Medical Center Göttingen, Germany)

- Tutor of the course 'Data Literacy' (Georg-August University Göttingen, Germany)

- Supervision of students 


\section{Professional skills}

- Brain stimulation techniques. The primary experience is in transcranial magnetic stimulation of the human brain. I performed research projects to study the functional dynamics of ongoing brain activity.

- Data analysis. I have experience in the processing of time-series data such as EEG (electroencephalography) and fMRI (functional magnetic resonance images), and analysis of MRI (magnetic resonance images).

- Computational modeling. I performed the estimations of the electric field distribution of TMS pulses in the MRI-based human brain models.

\section{Additional skills}

- Neuroscientific tools: MRI and fMRI analysis: SPM, Conn, Freesurfer (limited knowledge); EEG analysis: EEGLAB, Fieldtrip; Computational modeling: Freesurfer and SimNIBS software.

- Software: Windows and Linux operating systems; MATLAB; Python: Scikit-learn, Pandas, NumPy; Inkscape software for visualization; general use of office suites.

- Designing, Building, and Operating Complex Equipment: extensively experienced with data acquisition from multiple neuroscientific modalities: EEG, TMS, Neuronavigation, and EMG.

- Management and Administration

- Data Collection and Analysis

- Teaching, Tutoring, and Presenting

- Language skills: Russian (native), English (oral and writing skills), German (intermediate level)

\section{Publications}

- Zmeykina E, Turi Z, Antal A, Paulus W. Inhibiting corticospinal excitability by entraining ongoing $\mu$-alpha rhythm in the motor cortex. bioRxiv 2020.11.11.378117; doi: https://doi.org/10.1101/2020.11.11.378117, 2020; 
- Zmeykina E, Mittner M, Paulus W, Turi Z. Short-lived alpha power suppression induced by low-intensity arrhythmic rTMS. bioRxiv 2020.10.28.358986; doi: https://doi.org/10.1101/2020.10.28.358986, 2020;

- Zmeykina E, Mittner M, Paulus W, Turi Z. Weak rTMS-induced electric fields produce neural entrainment in humans. Scientific Reports, 2020;

- Kremneva E, Sergeev D, Zmeykina E, Legostaeva L, Piradov M. Chronic disorders of consciousness: role of neuroimaging. Journal of Physics: Conference Series, 2017;

- Poydasheva A, Zmeykina E, Chernyavskiy A, Chervyakov A, Lykova J, Suponeva N, Piradov M. The efficacy of repetitive transcranial magnetic stimulation of left DLPFC with personalized targeting in major depression: preliminary results of pseudorandomized study. Brain Stimulation 2017

- Saenko I, Morozova S, Zmeykina E, Konovalov R, Chervyakov A, Poydasheva A, Chernikova L, Suponeva N, Piradov M, Kozlovskaya I. Changes in functional connectivity of motor zones in the course of treatment with a regent multimodal complex exoskeleton in neurorehabilitation of post-stroke patients. Fiziologiya Cheloveka, 2016

- Chervyakov A, Poydasheva A, Nazarova M, Zmeykina E, Suponeva N, Gnezditsky V, Chernikova L, Piradov M. Navigated repetitive transcranial magnetic stimulation in stroke rehabilitation (randomized double-blind sham-controlled study). Brain Stimulation, 2015 